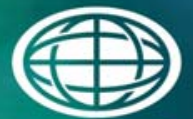

Savannah River

National Laboratory "m

OPERATED BY SAVANNAH RIVER NUCLEAR SOLUTIONS

\title{
Review of Rheology Modifiers for Hanford Waste
}

J. M Pareizs

September 2013

SRNL-STI-2013-00493 


\section{DISCLAIMER}

This work was prepared under an agreement with and funded by the U.S. Government. Neither the U.S. Government or its employees, nor any of its contractors, subcontractors or their employees, makes any express or implied:

1. warranty or assumes any legal liability for the accuracy, completeness, or for the use or results of such use of any information, product, or process disclosed; or

2. representation that such use or results of such use would not infringe privately owned rights; or

3. endorsement or recommendation of any specifically identified commercial product, process, or service.

Any views and opinions of authors expressed in this work do not necessarily state or reflect those of the United States Government, or its contractors, or subcontractors.

\section{Printed in the United States of America \\ Prepared for U.S. Department of Energy}


Retention: Permanent

\section{Review of Rheology Modifiers for Hanford Waste}

J. M. Pareizs

September 2013

Prepared for the U.S. Department of Energy under contract number DE-AC09-08SR22470.

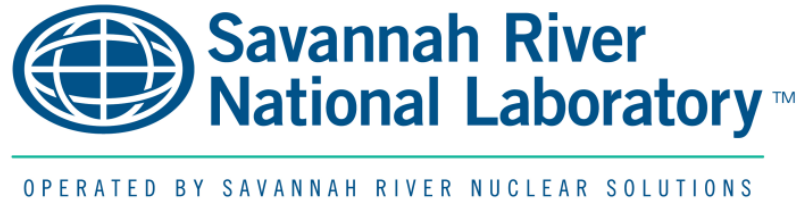




\section{REVIEWS AND APPROVALS}

\section{AUTHORS:}

J. M. Pareizs, Process Technology Programs

Date

TECHNICAL REVIEW:

M. E. Stone, Process Technology Programs

Date

\section{APPROVAL:}

C. C. Herman, SRNL Hanford Program Manager

Date

Environmental \& Chemical Process Technology Research Programs

S. L. Marra, Manager

Date

Environmental \& Chemical Process Technology Research Programs 
SRNL-STI-2013-00493

Revision 0

\section{ACKNOWLEDGEMENTS}

Funding for this report was provided by the Department of Energy - Office of River Protection under Inter-Entity Work Order (IEWO) M0SRV00091. 


\section{EXECUTIVE SUMMARY}

As part of Savannah River National Laboratory (SRNL)'s strategic development scope for the Department of Energy - Office of River Protection (DOE-ORP) Hanford Tank Waste Treatment and Immobilization Plant (WTP) waste feed acceptance and product qualification scope, the SRNL has been requested to recommend candidate rheology modifiers to be evaluated to adjust slurry properties in the Hanford Tank Farm. SRNL has performed extensive testing of rheology modifiers for use with Defense Waste Processing Facility (DWPF) simulated melter feed - a high undissolved solids (UDS) mixture of simulated Savannah River Site (SRS) Tank Farm sludge, nitric and formic acids, and glass frit. A much smaller set of evaluations with Hanford simulated waste have also been completed. This report summarizes past work and recommends modifiers for further evaluation with Hanford simulated wastes followed by verification with actual waste samples.

Based on the review of available data, a few compounds/systems appear to hold the most promise. For all types of evaluated simulated wastes (caustic Handford tank waste and DWPF processing samples with $\mathrm{pH}$ ranging from slightly acidic to slightly caustic), polyacrylic acid had positive impacts on rheology. Citric acid also showed improvement in yield stress on a wide variety of samples.

It is recommended that both polyacrylic acid and citric acid be further evaluated as rheology modifiers for Hanford waste. These materials are weak organic acids with the following potential issues:

- The acidic nature of the modifiers may impact waste $\mathrm{pH}$, if added in very large doses. If $\mathrm{pH}$ is significantly reduced by the modifier addition, dissolution of UDS and increased corrosion of tanks, piping, pumps, and other process equipment could occur. Smaller shifts in $\mathrm{pH}$ could reduce aluminum solubility, which would be expected to increase the yield stress of the sludge. Therefore, it is expected that use of an acidic modifier would be limited to concentrations that do not appreciably change the $\mathrm{pH}$ of the waste.

- Organics are typically reductants and could impact glass REDOX if not accounted for in the reductant addition calculations.

- Stability of the modifiers in a caustic, radioactive environment is not known, but some of the modifiers tested were specifically designed to withstand caustic conditions.

- These acids will add to the total organic carbon content of the wastes. Radiolytic decomposition of the acids could result in organic and hydrogen gas generation.

These potential impacts must be addressed in future studies with simulants representative of real waste and finally with tests using actual waste based on the rheology differences seen between SRS simulants and actual waste. The only non-organic modifier evaluated was sodium metasilicate. Further evaluation of this modifier is recommended if a reducing modifier is a concern. 


\section{TABLE OF CONTENTS}

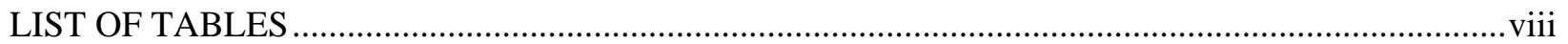

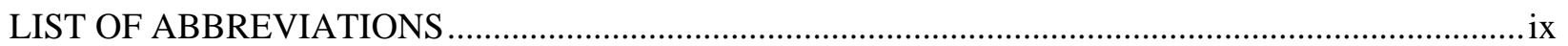

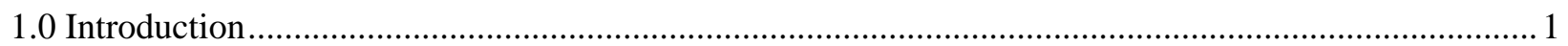

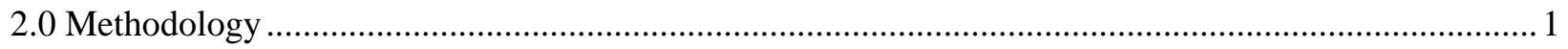

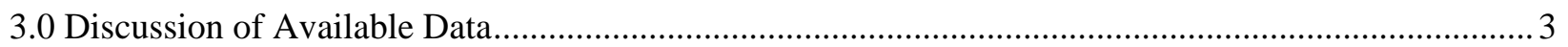

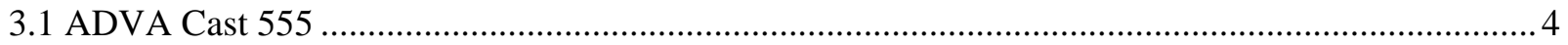

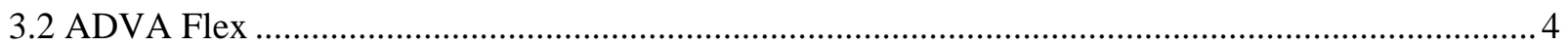

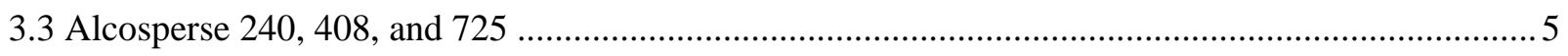

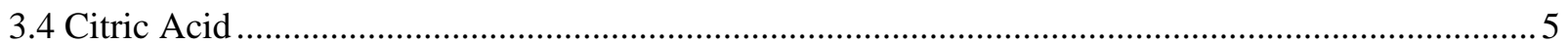

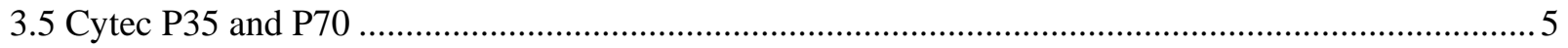

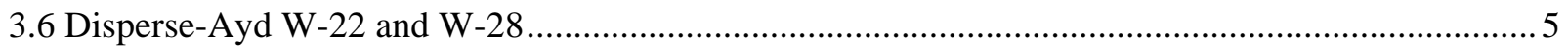

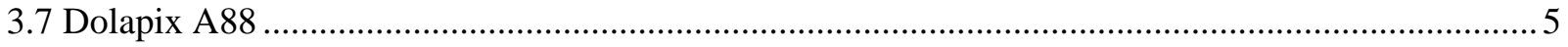

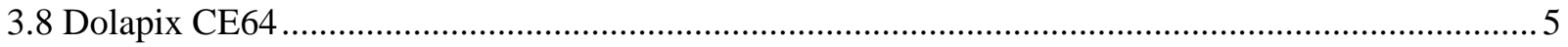

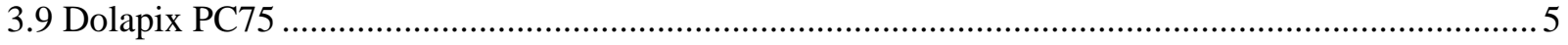

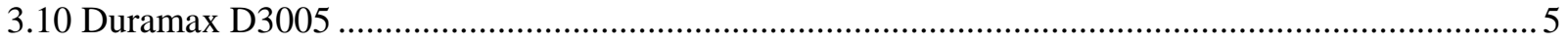

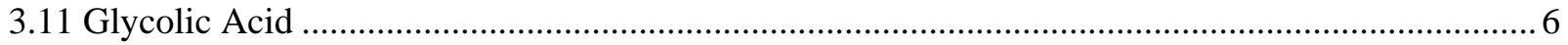

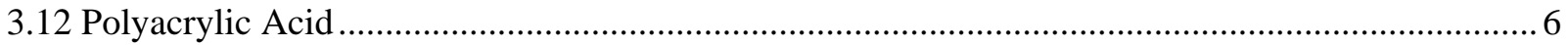

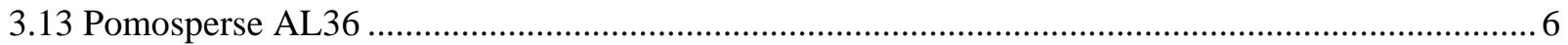

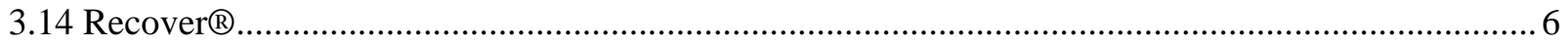

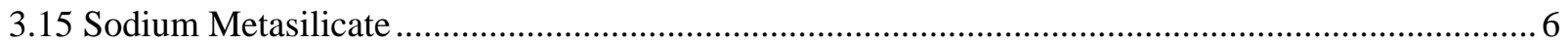

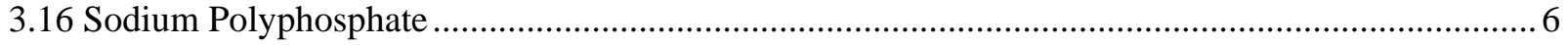

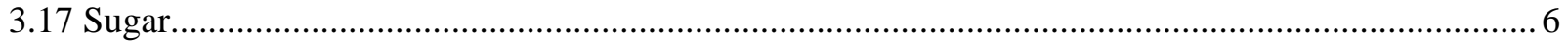

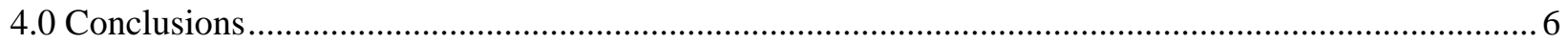

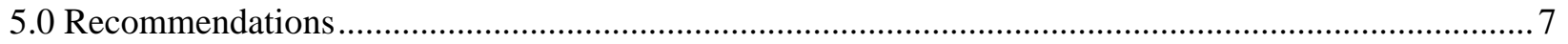

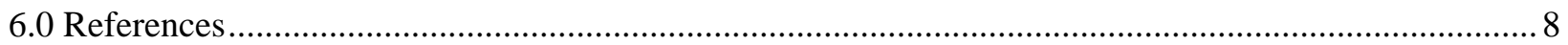

Appendix A . Rheology Results: Summaries From Referenced Reports ..............................................A-1

Appendix B . Evaluated Rheological Modifiers: Excerpts From Reference Documents ........................ B-1 
SRNL-STI-2013-00493

Revision 0

\section{LIST OF TABLES}

Table 2-1. Description of Simulants Used in Modifier Testing................................................................ 2

Table 2-2. Modifiers That Resulted in a 10\% Decrease in Yield Stress ................................................... 3 


\section{LIST OF ABBREVIATIONS}

$\begin{array}{ll}\text { DOE-ORP } & \text { Department of Energy - Office of River Protection } \\ \text { DWPF } & \text { Defense Waste Processing Facility } \\ \text { PNNL } & \text { Pacific Northwest National Laboratory } \\ \text { SME } & \text { Slurry Mix Evaporator } \\ \text { SRAT } & \text { Sludge Receipt Adjustment Tank } \\ \text { SRNL } & \text { Savannah River National Laboratory } \\ \text { SRS } & \text { Savannah River Site } \\ \text { UDS } & \text { Undissolved solids } \\ \text { WTP } & \text { Hanford Tank Waste Treatment and Immobilization Plant }\end{array}$




\subsection{Introduction}

The Savannah River National Laboratory (SRNL) and the Pacific Northwest National Laboratory (PNNL) were tasked to jointly coordinate the engagement of the broader national laboratory community to solve technological challenges in initiative areas related to the Hanford tank wastes program. One of the focus areas was Waste Feed Acceptance and Product Qualification. Working with the Department of Energy - Office of River Protection (DOE-ORP) and Hanford contractor personnel, the laboratories completed a preliminary assessment of the technology gaps and potential areas for improvement in this area earlier in the fiscal year. Technical report, SRNL-STI-2012-00776/PNNL-22116, ${ }^{1}$, was issued to document this assessment. The use of rheological modifiers as a potential means for altering the rheological properties of Hanford wastes was identified in this gap assessment. The Hanford Tank Waste Treatment and Immobilization Plant (WTP) Pretreatment facility currently has a very low rheology limit (yield stress and consistency) in the WAC for WTP transfers from the Tank Farm to the Pretreatment facility. To meet this limit, rheological modifiers may be necessary and must be effective in high $\mathrm{pH}$ environments. Even if the limit is increased, rheological modifiers may still be necessary depending on the properties of some of the Hanford wastes or they may provide a more attractive option than dilution through water addition.

In an April meeting between DOE-ORP, the Hanford contractors, and the laboratories, the laboratories were requested to recommend candidate rheology modifiers to be evaluated for use in the Hanford Tank Farm. The work scope is described in a SRNL Task Technical \& Quality Assurance Plan. ${ }^{2}$

SRNL has performed extensive testing of rheology modifiers for use with Defense Waste Processing Facility (DWPF) simulated melter feed - a high undissolved solids (UDS) mixture of simulated Savannah River Site (SRS) Tank Farm sludge, nitric and formic acids, and glass frit. ${ }^{3-6}$ A much smaller set of evaluations with Hanford simulated tank waste has also been completed (simulated Hanford tanks AZ-101 and AZ-102, some samples pretreated with a Cs ion exchange resin). ${ }^{5,7,8}$ A review of available literature and data has been performed for applicability to Hanford tank waste conditions. Evaluated simulated wastes ranged from slightly acidic to pH 12 . This report summarizes past work and recommends modifiers for further evaluation with Hanford simulated wastes.

\subsection{Methodology}

Available literature on the effect of rheological modifiers on Savannah River Site and Hanford simulated wastes were reviewed. ${ }^{3-6,8}$ Evaluations of modifiers at elevated temperatures was published, ${ }^{4}$ but those results are not considered in this report as the conditions of interest were ambient temperature. A spreadsheet of the available results was created (see Appendix A). In these reports, three main categories of samples were evaluated: DWPF simulated melter feed, DWPF simulated Sludge Receipt and Adjustment Tank (SRAT) product (i.e., chemical processed feed), and Hanford simulated waste. A summary description of these categories is presented in Table 2-1. 
Table 2-1. Description of Simulants Used in Modifier Testing

\begin{tabular}{|c|c|c|}
\hline "Sample Category & Description & Ref. \\
\hline $\begin{array}{l}\text { DWPF simulated SRAT } \\
\text { Product Simulants (i.e., } \\
\text { chemical processed feed) }\end{array}$ & $\begin{array}{l}\text { SRS sludge with added formic and nitric acids (primarily for } \\
\text { reduction of mercury and destruction of nitrite) } \\
\text { Heated to boiling to facilitate reactions and concentrate solids } \\
\text { Typical pH }<7 \\
\text { Typical wt } \% \text { UDS } 10-20 \%\end{array}$ & 6 \\
\hline $\begin{array}{l}\text { DWPF Simulated Melter } \\
\text { Feed }\end{array}$ & $\begin{array}{l}\text { SRS SRAT material with added frit (glass formers) } \\
\text { Heated to boiling to concentrate solids } \\
\text { Typical pH }<7 \\
\text { Typical wt } \% \text { UDS } 35-50 \%\end{array}$ & $3-5,8$ \\
\hline \multirow{2}{*}{$\begin{array}{l}\text { Hanford Simulated Waste } \\
\text { (Tanks AZ-101/102) }\end{array}$} & $\begin{array}{l}\text { Hanford precipitated Tank AZ-101 simulants (precipitation via } \\
\text { pretreatment with Cs ion exchange concentrate) } \\
\text { pH 10-12 } \\
\text { wt\% UDS not published }\end{array}$ & 7,8 \\
\hline & $\begin{array}{l}\text { Hanford Tank AZ-102 simulant } \\
\text { pH not published } \\
\text { wt\% UDS } 12.1 \%\end{array}$ & 5 \\
\hline
\end{tabular}

For most of the studies, rheological measurements (specifically yield stress) of samples with modifier were compared to results with no modifier. Samples and modifiers which resulted in a $10 \%$ or greater decrease in yield stress were screened. That is, if a modifier did not decrease yield stress by at least $10 \%$ in any evaluation, it was removed from this evaluation. Examples of modifiers that did not meet this criterion are antifoams, which are typically used in processing to mitigate foaming. The results of this screening are presented in Table 2-2. Included in the table are brief descriptions of the modifiers. Excerpts from the source reports, with more detailed descriptions, are given in Appendix B. Over 90 percent of the remaining data for screening involved DWPF simulated melter feed. 
Table 2-2. Modifiers That Resulted in a $10 \%$ Decrease in Yield Stress

\begin{tabular}{|c|c|c|c|c|}
\hline Modifier & Description & $\begin{array}{c}\text { DWPF } \\
\text { Simulated } \\
\text { SRAT } \\
\text { Product }\end{array}$ & $\begin{array}{c}\text { DWPF } \\
\text { Simulated } \\
\text { Melter Feed }\end{array}$ & $\begin{array}{c}\text { Hanford } \\
\text { Simulated } \\
\text { Waste }\end{array}$ \\
\hline ADVA Cast 555 & organic mixture $^{\text {a }}$ & Not Evaluated & $\mathrm{X}$ & Not evaluated \\
\hline ADVA Flex & $\begin{array}{c}\text { proprietary } \\
\text { polycarboxylate, }\end{array}$ & Not Evaluated & $\mathrm{X}$ & Not evaluate \\
\hline Alcosperse 149 & sodium polyacrylate & Not Evaluated & Not Evaluated & $\mathrm{X}$ \\
\hline Alcosperse 240 & $\begin{array}{l}\text { proprietary } \\
\text { polyacrylate }\end{array}$ & Not Evaluated & Not Evaluated & $\mathrm{X}$ \\
\hline Alcosperse 408 & $\begin{array}{l}\text { proprietary } \\
\text { polyacrylate }\end{array}$ & Not Evaluated & Not Evaluated & $\mathrm{X}$ \\
\hline Alcosperse 725 & $\begin{array}{l}\text { proprietary } \\
\text { polyacrylate }\end{array}$ & Not Evaluated & Not Evaluated & $\mathrm{X}$ \\
\hline Citric Acid & citric acid & Not Evaluated & $\mathrm{X}$ & $\mathrm{X}$ \\
\hline Cytec P35 & $\begin{array}{l}\text { proprietary } \\
\text { polyacrylate }\end{array}$ & Not Evaluated & Not Evaluated & $\mathrm{X}$ \\
\hline Cytec P70 & $\begin{array}{l}\text { proprietary } \\
\text { polyacrylate }\end{array}$ & Not Evaluated & Not Evaluated & $\mathrm{X}$ \\
\hline Disperse-Ayd W-28 & $\begin{array}{l}\text { proprietary } \\
\text { polyacrylate }\end{array}$ & $\mathrm{X}$ & $\mathrm{X}$ & $\begin{array}{c}\text { Yield stress } \\
\text { increased }\end{array}$ \\
\hline Dolapix A88 & organic mixture & Not Evaluated & $\mathrm{X}$ & Not evaluated \\
\hline Dolapix CE64 & $\begin{array}{l}\text { proprietary } \\
\text { polyacrylate }\end{array}$ & $\mathrm{X}$ & $\mathrm{X}$ & $\mathrm{X}$ \\
\hline Dolapix PC75 & $\begin{array}{c}\text { synthetic } \\
\text { polyelectrolyte }\end{array}$ & Not Evaluated & $\mathrm{X}$ & Not evaluated \\
\hline Duramax 3005 & polyglycol & Not Evaluated & Not Evaluated & $\mathrm{X}$ \\
\hline Glycolic Acid Solution & glycolic acid & Not Evaluated & $\mathrm{X}$ & Not Evaluated \\
\hline Polyacrylic Acid & polyacrylic acid & Not Evaluated & $\mathrm{X}$ & $\mathrm{X}$ \\
\hline Pomosperse AL36 & $\begin{array}{c}\text { proprietary } \\
\text { polyacrylate }\end{array}$ & Not Evaluated & Not Evaluated & $\mathrm{X}$ \\
\hline Recover $^{\circledR}$ & organic mixture $^{\mathrm{b}}$ & Not Evaluated & $\mathrm{X}$ & Not Evaluated \\
\hline Sodium Metasilicate & crystallized silicate & Not Evaluated & $\mathrm{X}$ & Not Evaluated \\
\hline Sodium Polyphosphate & phosphate polymer & Not Evaluated & Not Evaluated & $\mathrm{X}$ \\
\hline Sugar & glucose & Not Evaluated & $\mathrm{X}$ & Not Evaluated \\
\hline
\end{tabular}

\subsection{Discussion of Available Data}

A review of several rheology modifier reports was completed and a screening of the effectiveness and potential applicability was completed. Based on this review, polyacrylic acid appeared to be most applicable for use with Hanford wastes. This modifier had positive impact on rheology for Hanford simulated waste and the relatively higher insoluble solids DWPF simulated melter feeds. Citric acid should also be investigated. Results with Hanford simulated waste with citric acid are comparable to the results with polyacrylic acid. Most tests with DWPF simulated melter feed and citric acid showed improvement in yield stress. For both modifiers, good results (greater than 
$40 \%$ reduction in yield stress) were observed with additions of less than 10,000 ppm (or 1\%). Note that simple dilution of a sample with water will decrease yield stress, but added water will ultimately need to be removed. Thus, a low concentration of modifier is desirable.

Both polyacrylic and citric acids are weak acids and may impact $\mathrm{pH}$ and dissolution of UDS of caustic tank waste. Both acids are organic and may act as reductants in waste processing. These potential impacts must be addressed in future studies. The only non- organic modifier evaluated was sodium metasilicate. Further evaluation of this modifier is recommended if a reducing modifier is a concern.

Following are excerpts and brief discussion of the conclusions from the referenced reports.

In a study of several rheology modifiers with Hanford simulated waste, results, citric acid and polyacrylic acid improved rheology: ${ }^{7}$ " ...it was found that weak acid-type modifiers are much more efficient. Among the weak acid-type modifiers, CA [citric acid] and PAA [polyacrylic acid] turned out to be two of the most efficient rheological modifiers, reducing yield stresses by about $70 \%$ at 5000 ppm.”

From Hansen ${ }^{3}$ : "The results from this testing indicate that citric acid or polycarboxylate based rheology modifiers are the most effective in reducing the yield stress, by as much as $70 \%$ at the higher rheology modifier additions and were effective on most of the tested simulants." In a continuation of this work, in 2011" : "The most effective rheology modifiers were the polyacrylic series of modifiers, reducing yield stress as much as $90 \%$, but they did have one drawback, they made the SME [Slurry Mix Evaporator] product [i.e., melter feed] much more adhesive in appearance as compared to the other modifiers and baseline conditions." In these studies, all samples tested were DWPF simulated melter feed samples. The rheology modifiers are organic, hence they can also be considered reductants in melter operations. Some modifiers are also acids and may impact aspects of pretreatment (i.e., undesirable dissolution of metals or radionuclides).

From the $\mathrm{Kay}^{5}$ and Stone ${ }^{6,8}$ studies which included Hanford simulated wastes, two modifiers decreased yield stress by more than 30\% - Alcosperse 240, a polyacrylate, and Dolapix CE64, an ethylene glycol. In the Stone studies with DWPF simulants (both melter feed and SRAT product), ${ }^{6}$ Dolapix CE64, and Disperse-Ayd W28, a polyacrylate showed greater than 30\% improvement in yield stress. However, in another study with DWPF simulated melter feed, Dolapic CE64 increased yield stress. ${ }^{3}$

Following are summaries of modifier results. More detailed results of the modifier tests can be found in Appendix A, and detailed modifier descriptions can be found in Appendix B.

\subsection{ADVA Cast 555}

ADVA Cast 555, an organic mixture (see Appendix B), was evaluated (16 tests) with simulated DWPF melter feed at concentrations from 1,000 to 16,000 ppm. ${ }^{3}$ Yield stress decreased in most evaluations. Additions of greater than $10,000 \mathrm{ppm}$ yielded greater than $50 \%$ reduction with several samples.

\subsection{ADVA Flex}

This modifier, a polycarboxylate, was tested with several DWPF simulated melter feed samples at concentrations from nominally 1,000 to $16,000 \mathrm{ppm}^{3}$ Of the 15 tests, 6 had yield stress decreases of $50 \%$. However, over 10,000 ppm of modifier was used for these results. 


\subsection{Alcosperse 240,408 , and 725}

These modifiers, all polyacrylate based, were used at a concentration of 1,000 ppm with Hanford waste simulants. ${ }^{8}$ With a total of 8 tests, one showed improvement of $2 \%$, while the remaining samples ranged from 14 to $37 \%$.

\subsection{Citric Acid}

Eighty runs of various DWPF simulated melter feed samples were completed with citric acid concentrations varying between 1,000 and 10,000 ppm., ${ }^{3,4}$ Of all the runs, 33 showed less than a $10 \%$ improvement or an increase in yield stress. Twenty-four runs showed improvement of greater than 30\%. Greatest improvements in yield stress occurred at concentrations of 4,000 to 16,000 ppm citric acid. In a study with Hanford simulated waste, a decrease in yield stress of greater than $30 \%$ was observed with $1,000 \mathrm{ppm}$ citric acid and a decrease of nearly $70 \%$ at a concentration of $5,000 \mathrm{ppm}^{7}$

\subsection{Cytec P35 and P70}

The Cytec modifiers, both polyacrylate based, were evaluated with Hanford waste simulants at concentrations of $1,000 \mathrm{ppm} .{ }^{8}$ In all cases (4 runs -2 simulants, 2 modifiers), yield stress improved between 4 and 30\%.

\subsection{Disperse-Ayd W-22 and W-28}

These modifiers, both polyacrylate based, were tested with Hanford waste simulant, DWPF simulated melter feed, and DWPF simulated SRAT product. ${ }^{5,6}$ Twelve samples were evaluated with concentrations between 500 and 9,000 ppm. Yield stress was impacted positively in most cases with concentrations of 2,000 ppm or greater. Of the seven samples with 1,000 ppm or less addition, only one had a decrease in yield stress of greater than $10 \%$.

\subsection{Dolapix A88}

This modifier, an organic mixture (see Appendix B), was tested with DWPF simulated melter feed. ${ }^{3}$ Of the 13 tests, only 2 showed a decrease of greater than $10 \%$ in yield stress, while yield stress increased significantly with 10 evaluations. Concentrations ranged from 800 to 10,000 ppm. As modifier concentration increased, yield stress increased.

\subsection{Dolapix CE64}

This modifier, a polyacrylate, was tested with 32 DWPF simulated SRAT product and melter feed samples at concentrations from 600 to 16,000 ppm., 6 Yield stress showed improvement in approximately two thirds of the evaluations with no correlation to modifier concentration.

\subsection{Dolapix PC75}

This modifier, a synthetic polyelectrolyte, was evaluated 12 times with DWPF simulated melter feed at concentrations from 1,000 to 16,000 ppm. ${ }^{3}$ Yield stress decreased in 8 of the evaluations. There was a slight correlation between yield stress improvement and modifier concentration.

\subsection{Duramax D3005}

This modifier, a polyglycol, was used with three Hanford simulated wastes and one DWPF melter feed sample at 1,000 ppm. ${ }^{5,8}$ The modifier reduced yield stress by more than $10 \%$ for the Hanford simulants, but significantly increased yield stress for the DWPF melter feed simulant. 


\subsection{Glycolic Acid}

Glycolic acid was evaluated with 52 combinations of DWPF simulated melter feed at concentrations from 1,000 to $14,000 \mathrm{ppm} .{ }^{4}$ Yield stress decreased by $10 \%$ in only 14 of the tests. It should be noted that glycolic acid is currently being considered as the replacement acid/reductant in DWPF processing. It has been shown to provide consistent reduction in the yield stress of sludge during the DWPF chemical processing and melter feed preparation steps, but at much higher concentrations than those evaluated in testing as a rheological modifier.

\subsection{Polyacrylic Acid}

Polyacrylic acids at varying molecular weights and concentrations in the samples ranging between 900 and 14,000 ppm were evaluated with DWPF simulated melter feed (188 evaluations). ${ }^{3,4}$ A decrease in yield stress was observed in 161 of the tests. Reduction in yield stress also appears to correlate with concentration of modifier. In a study with Hanford simulated waste, yield stress decreased by over $50 \%$ as the modifier concentration was increased to 5,000 ppm. ${ }^{1}$

\subsection{Pomosperse AL36}

This polyacrylate based modifier was tested on two Hanford simulated wastes. ${ }^{8}$ Yield stress decreased by 9 and $14 \%$.

\section{$3.14 \underline{\text { Recover }}$}

Recover ${ }^{\circledR}$, an organic mixture (see Appendix B for composition), was evaluated 76 times at concentrations from 1,000 to 16,000 ppm with DWPF simulated melter feed. ${ }^{3,4}$ In 14 trials, yield stress either was not affected or increased.

\subsection{Sodium Metasilicate}

Sodium metasilicate was evaluated four times with DWPF simulated melter feed at concentrations from 1,000 to 10,000 ppm. ${ }^{3}$ Yield stress improved in all four evaluations, from 3 to $19 \%$.

\subsection{Sodium Polyphosphate}

This modifier was evaluated 13 times with DWPF simulated melter feed and Hanford simulated waste at 1,000 to $10,000 \mathrm{ppm} .{ }^{3,8}$ Yield stress improved in only three evaluations.

\subsection{7 $\underline{\text { Sugar }}$}

Sugar was evaluated four times with DWPF simulated melter feed at concentrations from 1,000 to $10,000 \mathrm{ppm}^{3}$ Yield stress improved in all four evaluations, from 2 to $17 \%$.

\subsection{Conclusions}

Based on the limited data available, the weak acids polyacrylic acid and citric acid positively affected rheology of Hanford simulated wastes. These materials also showed improvement with relatively higher UDS DWPF simulated melter feed. The only non-organic modifier, sodium metasilicate also showed potential for decreasing yield stress. 


\subsection{Recommendations}

It is recommended that both polyacrylic acid and citric acid be further evaluated as rheology modifiers for Hanford waste. These materials are weak organic acids with the following potential issues:

- The acidic nature of the modifiers may impact waste $\mathrm{pH}$, if added in very large doses. If $\mathrm{pH}$ is significantly reduced by the modifier addition, dissolution of UDS and increased corrosion of tanks, piping, pumps, and other process equipment could occur. Smaller shifts in $\mathrm{pH}$ could reduce aluminum solubility, which would be expected to increase the yield stress of the sludge. Therefore, it is expected that use of an acidic modifier would be limited to concentrations that do not appreciably change the $\mathrm{pH}$ of the waste.

- Organics are typically reductants and could impact glass REDOX if not accounted for in the reductant addition calculations.

- Stability of the modifiers in a caustic, radioactive environment is not known, but some of the modifiers tested were specifically designed to withstand caustic conditions.

- These acids will add to the total organic carbon content of the wastes. Radiolytic decomposition of the acids could result in organic and hydrogen gas generation.

These potential impacts must be addressed in future studies with simulants representative of real waste and finally with tests using actual waste based on the rheology differences seen between SRS simulants and actual waste. The only non-organic modifier evaluated was sodium metasilicate. Further evaluation of this modifier is recommended if a reducing modifier is a concern. 


\subsection{References}

1. Herman, C. C.; Peterson, R. A.; Adamson, D. J.; Herman, D. T.; Peeler, D. K.; Poirier, M. R.; Reboul, S. H.; Stone, M. E.; Chun, J.; Fort, J. A.; Vienna, J. D.; Wells, B. E. Preliminary Assessment of the Hanford Tank Waste Feed Acceptance and Product Qualification Programs”, ; SRNL-STI-2012-00776/PNNL-22116; Savannah River National Laboratory: Aiken, SC, 2013.

2. Herman, C. C.; Reboul, S. H. Task Technical and Quality Assurance Plan for Strategic Initiatives Related to Hanford Waste Treatment and Immobilization Plant (WTP) Feed Acceptance and Qualification Strategy; SRNL-RP-2013-00364; Savannah River National Laboratory: Aiken, SC, 2013.

3. Hansen, E. K. Summary of 2009 Rheology Modifier Program; SRNL-STI-2009-00697; Savannah River National Laboratory: Aiken, SC, 2009.

4. Hansen, E. K. 2011 EM/SRNL Rheology Modifiers Summary Report; SRNL-STI-201100670; Savannah River National Laboratory: Aiken, SC, 2011.

5. Kay, E. D.; T. B. Calloway, J.; Koopman, D. C.; Brigmon, R. L.; Eibling, R. E. Rheology Modifiers for Radioactive Waste Slurries; WSRC-MS-2003-00136; Westinghouse Savannah River Company: Aiken, SC, 2003.

6. Stone, M. E.; Marinik, A. R.; Marsh, D. M. Rheological Modifier Testing with DWPF Process Slurries; WSRC-TR-2004-00082; Savannah River National Laboratory: Aiken, SC, 2004.

7. Chun, J.; Poloski, A. P.; Hansen, E. K., Stabilization and control of rheological properties of $\mathrm{Fe} 2 \mathrm{O} 3 / \mathrm{Al}(\mathrm{OH}) 3$-rich colloidal slurries under high ionic strength and $\mathrm{pH}$. Journal of Colloid and Interface Science 2010, 348, 280-288.

8. Stone, M. E. Summary of Rheologyical Modifier Testing on RPP Simulants; SRNL-GPD2004-0040; Savannah River National Laboratory: Aiken, SC, 2004. 
SRNL-STI-2013-00493

Revision 0

Appendix A. Rheology Results: Summaries From Referenced Reports 
The following table contains numerical results from references used in this report. The Applicable reference is given in the table.

UDS=undissolved solids

np=not published

Calc \% Change $=($ Yield Stress w Mod - Yield Stress no Mod $) /$ Yield Stress no Mod $)$

\begin{tabular}{|c|c|c|c|c|c|c|c|c|c|c|}
\hline Modifier & Sample & $\begin{array}{l}\text { Mod conc, } \\
\text { ppm }\end{array}$ & $\begin{array}{c}\text { Total Solids, } \\
\%\end{array}$ & UDS, \% & $\begin{array}{c}\text { Yield } \\
\text { Stress } \\
\text { no Mod, } \\
\text { Pa }\end{array}$ & $\begin{array}{c}\text { Yield } \\
\text { Stress w } \\
\text { Mod, Pa }\end{array}$ & $\begin{array}{l}\text { Calc \% } \\
\text { Change }\end{array}$ & pH & Ref & Category \\
\hline ADVA Cast 555 & $\begin{array}{l}\text { RuRhHg 1-9 Decanted } \\
\text { SME }\end{array}$ & 16,040 & np & np & 72.5 & 25.4 & -65.0 & np & SRNL-STI-2009-697 & DWPF Simulated Melter Feed \\
\hline ADVA CAST 555 & 08-SB5-12/13 SME & 11,189 & 44.7 & 34.4 & 6.6 & 2.32 & -64.8 & 6.27 & SRNL-STI-2009-697 & DWPF Simulated Melter Feed \\
\hline ADVA Cast 555 & $\begin{array}{l}\text { RuRhHg 1-9 Decanted } \\
\text { SME }\end{array}$ & 11,042 & np & np & 72.5 & 32.4 & -55.3 & np & SRNL-STI-2009-697 & DWPF Simulated Melter Feed \\
\hline ADVA CAST 555 & 08-SB5-12/13 SME & 7,099 & 44.7 & 34.4 & 6.6 & 3.53 & -46.5 & 6.27 & SRNL-STI-2009-697 & DWPF Simulated Melter Feed \\
\hline ADVA CAST 555 & 09-SB5-23 SME & 16,130 & 49.6 & 35.1 & 24.4 & 13.3 & -45.5 & 7.37 & SRNL-STI-2009-697 & DWPF Simulated Melter Feed \\
\hline ADVA CAST 555 & 09-SB5-23 SME & 11,082 & 49.6 & 35.1 & 24.4 & 15.4 & -36.9 & 7.37 & SRNL-STI-2009-697 & DWPF Simulated Melter Feed \\
\hline ADVA Cast 555 & $\begin{array}{l}\text { RuRhHg 1-9 Decanted } \\
\text { SME }\end{array}$ & 6,073 & np & np & 72.5 & 45.8 & -36.8 & np & SRNL-STI-2009-697 & DWPF Simulated Melter Feed \\
\hline ADVA CAST 555 & 09-SB5-24 SME & 11,189 & 48.8 & 34.5 & 5.22 & 3.42 & -34.5 & 5.14 & SRNL-STI-2009-697 & DWPF Simulated Melter Feed \\
\hline ADVA CAST 555 & 09-SB5-23 SME & 6,068 & 49.6 & 35.1 & 24.4 & 18.2 & -25.4 & 7.37 & SRNL-STI-2009-697 & DWPF Simulated Melter Feed \\
\hline ADVA CAST 555 & 09-SB5-24 SME & 6,070 & 48.8 & 34.5 & 5.22 & 3.94 & -24.5 & 5.14 & SRNL-STI-2009-697 & DWPF Simulated Melter Feed \\
\hline ADVA CAST 555 & 08-SB5-12/13 SME & 3,446 & 44.7 & 34.4 & 6.6 & 5.26 & -20.3 & 6.27 & SRNL-STI-2009-697 & DWPF Simulated Melter Feed \\
\hline ADVA CAST 555 & 09-SB5-24 SME & 16,397 & 48.8 & 34.5 & 5.22 & 4.25 & -18.6 & 5.14 & SRNL-STI-2009-697 & DWPF Simulated Melter Feed \\
\hline ADVA Cast 555 & $\begin{array}{l}\text { RuRhHg 1-9 Decanted } \\
\text { SME }\end{array}$ & 1,023 & np & np & 72.5 & 65.9 & -9.1 & 4.59 & SRNL-STI-2009-697 & DWPF Simulated Melter Feed \\
\hline ADVA CAST 555 & 09-SB5-24 SME & 1,008 & 48.8 & 34.5 & 5.22 & 4.78 & -8.4 & 5.14 & SRNL-STI-2009-697 & DWPF Simulated Melter Feed \\
\hline ADVA CAST 555 & 09-SB5-23 SME & 1,077 & 49.6 & 35.1 & 24.4 & 22.4 & -8.2 & 7.37 & SRNL-STI-2009-697 & DWPF Simulated Melter Feed \\
\hline ADVA CAST 555 & 08-SB5-12/13 SME & 1,121 & 44.7 & 34.4 & 6.6 & 6.65 & 0.8 & 6.27 & SRNL-STI-2009-697 & DWPF Simulated Melter Feed \\
\hline ADVA Flex & $\begin{array}{l}\text { RuRhHg 1-9 Decanted } \\
\text { SME }\end{array}$ & 16,011 & np & np & 72.5 & 21.7 & -70.1 & 4.59 & SRNL-STI-2009-697 & DWPF Simulated Melter Feed \\
\hline ADVA Flex & RuRhHg 1-9 SME & 16,080 & 51.55 & 42 & 13.4 & 4.1 & -69.4 & 4.7 & SRNL-STI-2009-697 & DWPF Simulated Melter Feed \\
\hline ADVA FLEX & 09-SB5-23 SME & 16,120 & 49.6 & 35.1 & 24.4 & 9.4 & -61.5 & 7.37 & SRNL-STI-2009-697 & DWPF Simulated Melter Feed \\
\hline ADVA Flex & $\begin{array}{l}\text { RuRhHg 1-9 Decanted } \\
\text { SME }\end{array}$ & 11,005 & np & np & 72.5 & 31 & -57.2 & 4.59 & SRNL-STI-2009-697 & DWPF Simulated Melter Feed \\
\hline ADVA Flex & RuRhHg 1-9 SME & 11,069 & 51.55 & 42 & 13.4 & 5.8 & -56.7 & 4.7 & SRNL-STI-2009-697 & DWPF Simulated Melter Feed \\
\hline ADVA FLEX & 09-SB5-23 SME & 11,007 & 49.6 & 35.1 & 24.4 & 12.1 & -50.4 & 7.37 & SRNL-STI-2009-697 & DWPF Simulated Melter Feed \\
\hline ADVA Flex & \begin{tabular}{|l|} 
RuRhHg 1-9 Decanted \\
SME
\end{tabular} & 5,994 & np & np & 72.5 & 42.9 & -40.8 & 4.59 & SRNL-STI-2009-697 & DWPF Simulated Melter Feed \\
\hline ADVA Flex & RuRhHg 1-9 SME & 6,023 & 51.55 & 42 & 13.4 & 8 & -40.3 & 4.7 & SRNL-STI-2009-697 & DWPF Simulated Melter Feed \\
\hline ADVA FLEX & 09-SB5-23 SME & 6,142 & 49.6 & 35.1 & 24.4 & 15.2 & -37.7 & 7.37 & SRNL-STI-2009-697 & DWPF Simulated Melter Feed \\
\hline
\end{tabular}




\begin{tabular}{|c|c|c|c|c|c|c|c|c|c|c|}
\hline Modifier & Sample & $\begin{array}{l}\text { Mod conc, } \\
\text { ppm }\end{array}$ & $\begin{array}{c}\text { Total Solids, } \\
\%\end{array}$ & UDS, \% & \begin{tabular}{|c|} 
Yield \\
Stress \\
no Mod, \\
Pa \\
\end{tabular} & $\begin{array}{c}\text { Yield } \\
\text { Stress w } \\
\text { Mod, Pa }\end{array}$ & $\begin{array}{l}\text { Calc \% } \\
\text { Change }\end{array}$ & pH & Ref & Category \\
\hline ADVA FLEX & 09-SB5-24 SME & 6,201 & 48.8 & 34.5 & 5.22 & 4.04 & -22.6 & 5.14 & SRNL-STI-2009-697 & DWPF Simulated Melter Feed \\
\hline ADVA FLEX & 09-SB5-24 SME & 11,100 & 48.8 & 34.5 & 5.22 & 4.29 & -17.8 & np & SRNL-STI-2009-697 & DWPF Simulated Melter Feed \\
\hline ADVA Flex & RuRhHg 1-9 SME & 1,115 & 51.55 & 42 & 13.4 & 11.1 & -17.2 & 4.7 & SRNL-STI-2009-697 & DWPF Simulated Melter Feed \\
\hline ADVA FLEX & $\begin{array}{l}\text { RuRhHg 1-9 Decanted } \\
\text { SME }\end{array}$ & 1,062 & np & np & 72.5 & 61.3 & -15.4 & np & SRNL-STI-2009-697 & DWPF Simulated Melter Feed \\
\hline ADVA FLEX & 09-SB5-23 SME & 1,068 & 49.6 & 35.1 & 24.4 & 21.6 & -11.5 & 7.37 & SRNL-STI-2009-697 & DWPF Simulated Melter Feed \\
\hline ADVA FLEX & 09-SB5-24 SME & 16,154 & 48.8 & 34.5 & 5.22 & 4.78 & -8.4 & $\mathrm{np}$ & SRNL-STI-2009-697 & DWPF Simulated Melter Feed \\
\hline ADVA FLEX & 09-SB5-24 SME & 1,035 & 48.8 & 34.5 & 5.22 & 4.99 & -4.4 & 5.14 & SRNL-STI-2009-697 & DWPF Simulated Melter Feed \\
\hline ADVA FLEX & Melter Feed & 1,000 & 47 & 41 & 10.3 & 30.3 & 194.2 & $6-7$ & WSRC-MS-2003-136 & DWPF Simulated Melter Feed \\
\hline Alcosperse 149 & FIU AZ-101 & 1,000 & np & np & 10.36 & 7.37 & -28.9 & 9.85 & SRNL-GPD-2004-40 & Hanford Simulated Waste \\
\hline Alcosperse 149 & AZ-101 & 1,000 & $\mathrm{np}$ & $\mathrm{np}$ & 3.22 & 2.77 & -14.0 & 12.23 & SRNL-GPD-2004-40 & Hanford Simulated Waste \\
\hline Alcosperse 240 & FIU AZ-101 & 1,000 & np & np & 10.36 & 6.5 & -37.3 & 9.85 & SRNL-GPD-2004-40 & Hanford Simulated Waste \\
\hline Alcosperse 240 & AZ-101 & 1,000 & np & np & 3.22 & 2.63 & -18.3 & 12.23 & SRNL-GPD-2004-40 & Hanford Simulated Waste \\
\hline Alcosperse 408 & AZ-101 & 1,000 & np & np & 3.22 & 2.48 & -23.0 & 12.23 & SRNL-GPD-2004-40 & Hanford Simulated Waste \\
\hline Alcosperse 408 & FIU AZ-101 & 1,000 & $\mathrm{np}$ & np & 10.36 & 8.14 & -21.4 & 9.85 & SRNL-GPD-2004-40 & Hanford Simulated Waste \\
\hline Alcosperse 725 & FIU AZ-101 & 1,000 & $\mathrm{np}$ & np & 10.36 & 8.2 & -20.8 & 9.85 & SRNL-GPD-2004-40 & Hanford Simulated Waste \\
\hline Alcosperse 725 & AZ-101 & 1,000 & np & np & 3.22 & 3.15 & -2.2 & 12.23 & SRNL-GPD-2004-40 & Hanford Simulated Waste \\
\hline Antifoam 747 & AZ102 simulant & 1,000 & 12.6 & 12.1 & 4.6 & 4.5 & -2.2 & np & WSRC-MS-2003-136 & Hanford Simulated Waste \\
\hline B52 antifoam & AZ102 simulant & 1,000 & 12.6 & 12.1 & 4.6 & 5.6 & 21.7 & $\mathrm{np}$ & WSRC-MS-2003-136 & Hanford Simulated Waste \\
\hline Citric Acid & SB6 SME-1 & 4,000 & 28.2 & 20.2 & 4.25 & 4.59 & 8.0 & np & SRNL-STI-2011-674 & DWPF Simulated Melter Feed \\
\hline Citric Acid & SB6 SME-1 & 1,000 & 28.2 & 20.2 & 4.25 & 4.56 & 7.3 & np & SRNL-STI-2011-674 & DWPF Simulated Melter Feed \\
\hline Citric Acid & SB6-1,2,3,4 SME & 10,035 & 46.9 & 37.2 & 2.2 & 2.33 & 5.9 & 6.02 & SRNL-STI-2009-697 & DWPF Simulated Melter Feed \\
\hline Citric Acid & SB6 SME-3 & 1,000 & 33 & 23.8 & 7.82 & 8.19 & 4.7 & $\mathrm{np}$ & SRNL-STI-2011-674 & DWPF Simulated Melter Feed \\
\hline Citric Acid & SB6 SME-1 & 7,000 & 28.2 & 20.2 & 4.25 & 4.37 & 2.8 & np & SRNL-STI-2011-674 & DWPF Simulated Melter Feed \\
\hline Citric Acid & SB6-1,2,3,4 SME & 1,050 & 46.9 & 37.2 & 2.2 & 2.26 & 2.7 & 6.02 & SRNL-STI-2009-697 & DWPF Simulated Melter Feed \\
\hline Citric Acid & SB6 SME-2 & 4,000 & 30.9 & 22.3 & 5.98 & 6.11 & 2.2 & $\mathrm{np}$ & SRNL-STI-2011-674 & DWPF Simulated Melter Feed \\
\hline Citric Acid & SB6 SME-9 & 1,000 & 48.2 & 36.4 & 31.2 & 31.8 & 1.9 & np & SRNL-STI-2011-674 & DWPF Simulated Melter Feed \\
\hline Citric Acid & SB6 SME-2 & 1,000 & 30.9 & 22.3 & 5.98 & 6.07 & 1.5 & nр & SRNL-STI-2011-674 & DWPF Simulated Melter Feed \\
\hline Citric Acid & SB6 SME-4 & 1,000 & 36 & 26.4 & 10.8 & 10.9 & 0.9 & $\mathrm{np}$ & SRNL-STI-2011-674 & DWPF Simulated Melter Feed \\
\hline Citric Acid & SB6-1,2,3,4 SME & 7,029 & 46.9 & 37.2 & 2.2 & 2.21 & 0.5 & 6.02 & SRNL-STI-2009-697 & DWPF Simulated Melter Feed \\
\hline Citric Acid & SB6 SME-9 & 4,000 & 48.2 & 36.4 & 31.2 & 31.1 & -0.3 & $\mathrm{np}$ & SRNL-STI-2011-674 & DWPF Simulated Melter Feed \\
\hline Citric Acid & SB6-1,2,3,4 SME & 3,523 & 46.9 & 37.2 & 2.2 & 2.16 & -1.8 & 6.02 & SRNL-STI-2009-697 & DWPF Simulated Melter Feed \\
\hline Citric Acid & SB6 SME-8 & 1,000 & 46.4 & 34.9 & 26.4 & 25.9 & -1.9 & np & SRNL-STI-2011-674 & DWPF Simulated Melter Feed \\
\hline Citric Acid & SB6 SME-3 & 4,000 & 33 & 23.8 & 7.82 & 7.67 & -1.9 & np & SRNL-STI-2011-674 & DWPF Simulated Melter Feed \\
\hline Citric Acid & SB6 SME-1 & 10,000 & 28.2 & 20.2 & 4.25 & 4.13 & -2.8 & np & SRNL-STI-2011-674 & DWPF Simulated Melter Feed \\
\hline Citric Acid & SB6 SME-7 & 1,000 & 43.6 & 32.4 & 24.9 & 24.1 & -3.2 & np & SRNL-STI-2011-674 & DWPF Simulated Melter Feed \\
\hline Citric Acid & SB6 SME-4 & 4,000 & 36 & 26.4 & 10.8 & 10.4 & -3.7 & $\mathrm{np}$ & SRNL-STI-2011-674 & DWPF Simulated Melter Feed \\
\hline Citric Acid & SB6 SME-2 & 7,000 & 30.9 & 22.3 & 5.98 & 5.74 & -4.0 & np & SRNL-STI-2011-674 & DWPF Simulated Melter Feed \\
\hline Citric Acid & SB6 SME-2 & 10,000 & 30.9 & 22.3 & 5.98 & 5.7 & -4.7 & nр & SRNL-STI-2011-674 & DWPF Simulated Melter Feed \\
\hline
\end{tabular}




\begin{tabular}{|c|c|c|c|c|c|c|c|c|c|c|}
\hline Modifier & Sample & $\begin{array}{l}\text { Mod conc, } \\
\text { ppm }\end{array}$ & $\begin{array}{c}\text { Total Solids, } \\
\%\end{array}$ & UDS, \% & $\begin{array}{c}\text { Yield } \\
\text { Stress } \\
\text { no Mod, } \\
\text { Pa } \\
\end{array}$ & $\begin{array}{c}\text { Yield } \\
\text { Stress w } \\
\text { Mod, Pa }\end{array}$ & $\begin{array}{l}\text { Calc \% } \\
\text { Change }\end{array}$ & $\mathbf{p H}$ & Ref & Category \\
\hline Citric Acid & SB6 SME-9 & 7,000 & 48.2 & 36.4 & 31.2 & 29.7 & -4.8 & np & SRNL-STI-2011-674 & DWPF Simulated Melter Feed \\
\hline Citric Acid & SB6 SME-7 & 4,000 & 43.6 & 32.4 & 24.9 & 23.6 & -5.2 & $\mathrm{np}$ & SRNL-STI-2011-674 & DWPF Simulated Melter Feed \\
\hline Citric Acid & SB6 SME-8 & 4,000 & 46.4 & 34.9 & 26.4 & 25 & -5.3 & np & SRNL-STI-2011-674 & DWPF Simulated Melter Feed \\
\hline Citric Acid & SB6 SME-5 & 1,000 & 38.6 & 28.3 & 14.5 & 13.7 & -5.5 & $\mathrm{np}$ & SRNL-STI-2011-674 & \begin{tabular}{|l} 
DWPF Simulated Melter Feed \\
\end{tabular} \\
\hline Citric Acid & SB6 SME-9 & 10,000 & 48.2 & 36.4 & 31.2 & 29.4 & -5.8 & np & SRNL-STI-2011-674 & \begin{tabular}{|l|} 
DWPF Simulated Melter Feed \\
\end{tabular} \\
\hline Citric Acid & SB6 SME-6 & 1,000 & 41.5 & 30.8 & 19 & 17.9 & -5.8 & np & SRNL-STI-2011-674 & DWPF Simulated Melter Feed \\
\hline Citric Acid & SB6 SME-4 & 10,000 & 36 & 26.4 & 10.8 & 10.1 & -6.5 & np & SRNL-STI-2011-674 & DWPF Simulated Melter Feed \\
\hline Citric Acid & SB6 SME-3 & 7,000 & 33 & 23.8 & 7.82 & 7.3 & -6.6 & np & SRNL-STI-2011-674 & DWPF Simulated Melter Feed \\
\hline Citric Acid & SB6 SME-6 & 4,000 & 41.5 & 30.8 & 19 & 17.7 & -6.8 & np & SRNL-STI-2011-674 & DWPF Simulated Melter Feed \\
\hline Citric Acid & SB6 SME-5 & 4,000 & 38.6 & 28.3 & 14.5 & 13.4 & -7.6 & np & SRNL-STI-2011-674 & DWPF Simulated Melter Feed \\
\hline Citric Acid & SB6 SME-4 & 7,000 & 36 & 26.4 & 10.8 & 9.9 & -8.3 & $\mathrm{np}$ & SRNL-STI-2011-674 & DWPF Simulated Melter Feed \\
\hline Citric Acid & SB6 SME-8 & 10,000 & 46.4 & 34.9 & 26.4 & 24 & -9.1 & $\mathrm{np}$ & SRNL-STI-2011-674 & DWPF Simulated Melter Feed \\
\hline Citric Acid & SB6 SME-7 & 10,000 & 43.6 & 32.4 & 24.9 & 22.5 & -9.6 & np & SRNL-STI-2011-674 & DWPF Simulated Melter Feed \\
\hline Citric Acid & SB6 SME-6 & 7,000 & 41.5 & 30.8 & 19 & 17.1 & -10.0 & np & SRNL-STI-2011-674 & DWPF Simulated Melter Feed \\
\hline Citric Acid & SB6 SME-3 & 10,000 & 33 & 23.8 & 7.82 & 7.02 & -10.2 & np & SRNL-STI-2011-674 & DWPF Simulated Melter Feed \\
\hline Citric Acid & SB6 SME-7 & 7,000 & 43.6 & 32.4 & 24.9 & 22.3 & -10.4 & np & SRNL-STI-2011-674 & DWPF Simulated Melter Feed \\
\hline Citric Acid & 09-SB5-24 SME & 10,054 & 48.8 & 34.5 & 5.22 & 4.65 & -10.9 & np & SRNL-STI-2009-697 & DWPF Simulated Melter Feed \\
\hline Citric Acid & SB6 SME-8 & 7,000 & 46.4 & 34.9 & 26.4 & 23.4 & $\begin{array}{c}-11.4 \\
\end{array}$ & np & SRNL-STI-2011-674 & DWPF Simulated Melter Feed \\
\hline Citric Acid & SB6 SME-5 & 7,000 & 38.6 & 28.3 & 14.5 & 12.7 & -12.4 & np & SRNL-STI-2011-674 & DWPF Simulated Melter Feed \\
\hline Citric Acid & SB6 SME-5 & 10,000 & 38.6 & 28.3 & 14.5 & 12.5 & -13.8 & np & SRNL-STI-2011-674 & DWPF Simulated Melter Feed \\
\hline Citric Acid & SB6 SME-6 & 10,000 & 41.5 & 30.8 & 19 & 16.3 & -14.2 & $\mathrm{np}$ & \begin{tabular}{|l|} 
SRNL-STI-2011-674 \\
\end{tabular} & DWPF Simulated Melter Feed \\
\hline Citric Acid & RuRhHg 1-9 SME & 9,996 & 51.55 & 42 & 13.4 & 11.4 & -14.9 & 4.7 & SRNL-STI-2009-697 & DWPF Simulated Melter Feed \\
\hline Citric Acid & 08-SB5-12/13 SME & 1,029 & 44.7 & 34.4 & 6.6 & 5.5 & -16.7 & 6.27 & SRNL-STI-2009-697 & DWPF Simulated Melter Feed \\
\hline Citric Acid & $\begin{array}{l}\text { RuRhHg 1-9 Decanted } \\
\text { SME }\end{array}$ & 7,020 & np & np & 72.5 & 59.6 & -17.8 & 4.59 & SRNL-STI-2009-697 & DWPF Simulated Melter Feed \\
\hline Citric Acid & $\begin{array}{l}\text { RuRhHg 1-9 Decanted } \\
\text { SME }\end{array}$ & 1,054 & np & np & 72.5 & 59.1 & -18.5 & 4.59 & SRNL-STI-2009-697 & DWPF Simulated Melter Feed \\
\hline Citric Acid & 09-SB5-24 SME & 7,018 & 48.8 & 34.5 & 5.22 & 4.24 & $\begin{array}{c}-18.8 \\
\end{array}$ & np & SRNL-STI-2009-697 & DWPF Simulated Melter Feed \\
\hline Citric Acid & $\begin{array}{l}\text { RuRhHg 1-9 Decanted } \\
\text { SME }\end{array}$ & 10,000 & np & np & 72.5 & 58.7 & -19.0 & 4.59 & SRNL-STI-2009-697 & DWPF Simulated Melter Feed \\
\hline Citric Acid & 09-SB5-23 SME & 1,055 & 49.6 & 35.1 & 24.4 & 19.4 & -20.5 & 7.37 & SRNL-STI-2009-697 & DWPF Simulated Melter Feed \\
\hline Citric Acid & SB4-SME-2 & 1,000 & 43.9 & 36 & 4.02 & 3.17 & -21.1 & $\mathrm{np}$ & SRNL-STI-2011-674 & DWPF Simulated Melter Feed \\
\hline Citric Acid & RuRhHg 1-9 SME & 7,006 & 51.55 & 42 & 13.4 & 10.4 & -22.4 & 4.7 & SRNL-STI-2009-697 & DWPF Simulated Melter Feed \\
\hline Citric Acid & RuRhHg 1-9 SME & 1,001 & 51.55 & 42 & 13.4 & 10.3 & -23.1 & 4.7 & SRNL-STI-2009-697 & DWPF Simulated Melter Feed \\
\hline Citric Acid & 09-SB5-24 SME & 1,049 & 48.8 & 34.5 & 5.22 & 3.96 & -24.1 & $\mathrm{np}$ & SRNL-STI-2009-697 & DWPF Simulated Melter Feed \\
\hline Citric Acid & RuRhHg 1-9 SME & 3,518 & 51.55 & 42 & 13.4 & 10.1 & -24.6 & 4.7 & SRNL-STI-2009-697 & DWPF Simulated Melter Feed \\
\hline Citric Acid & $\begin{array}{l}\text { RuRhHg 1-9 Decanted } \\
\text { SME }\end{array}$ & 3,506 & np & np & 72.5 & 52.8 & -27.2 & 4.59 & SRNL-STI-2009-697 & DWPF Simulated Melter Feed \\
\hline Citric Acid & 09-SB5-23 SME & 3,512 & 49.6 & 35.1 & 24.4 & 17.7 & -27.5 & 7.37 & SRNL-STI-2009-697 & DWPF Simulated Melter Feed \\
\hline
\end{tabular}




\begin{tabular}{|c|c|c|c|c|c|c|c|c|c|c|}
\hline Modifier & Sample & $\begin{array}{l}\text { Mod conc, } \\
\text { ppm }\end{array}$ & $\begin{array}{c}\text { Total Solids, } \\
\%\end{array}$ & UDS, \% & \begin{tabular}{|c|} 
Yield \\
Stress \\
no Mod, \\
Pa \\
\end{tabular} & \begin{tabular}{|c|} 
Yield \\
Stress w \\
Mod, Pa \\
\end{tabular} & $\begin{array}{l}\text { Calc \% } \\
\text { Change }\end{array}$ & $\mathbf{p H}$ & Ref & Category \\
\hline Citric Acid & 09-SB5-24 SME & 3,536 & 48.8 & 34.5 & 5.22 & 3.7 & -29.1 & $\mathrm{np}$ & SRNL-STI-2009-697 & DWPF Simulated Melter Feed \\
\hline Citric Acid & SB4-SME-5 & 1,000 & 51.9 & 42.5 & 23.8 & 15.7 & -34.0 & $\mathrm{np}$ & SRNL-STI-2011-674 & DWPF Simulated Melter Feed \\
\hline Citric Acid & 08-SB5-12/13 SME & 3,590 & 44.7 & 34.4 & 6.6 & 4.3 & -34.8 & 6.27 & SRNL-STI-2009-697 & DWPF Simulated Melter Feed \\
\hline Citric Acid & SB4-SME-4 & 1,000 & 49.2 & 40.1 & 13.5 & 8.3 & -38.5 & $\mathrm{np}$ & SRNL-STI-2011-674 & \begin{tabular}{|l|} 
DWPF Simulated Melter Feed \\
\end{tabular} \\
\hline Citric Acid & SB4-SME-1 & 1,000 & 41.2 & 33.7 & 3.05 & 1.83 & -40.0 & $\mathrm{np}$ & SRNL-STI-2011-674 & DWPF Simulated Melter Feed \\
\hline Citric Acid & 08-SB5-12/13 SME & 7,064 & 44.7 & 34.4 & 6.6 & 3.9 & -40.9 & 6.27 & SRNL-STI-2009-697 & DWPF Simulated Melter Feed \\
\hline Citric Acid & SB4-SME-3 & 1,000 & 46.7 & 38.4 & 7.49 & 4.39 & -41.4 & $\mathrm{np}$ & SRNL-STI-2011-674 & DWPF Simulated Melter Feed \\
\hline Citric Acid & SB4-SME-2 & 4,000 & 43.9 & 36 & 4.02 & 1.96 & -51.2 & np & SRNL-STI-2011-674 & DWPF Simulated Melter Feed \\
\hline Citric Acid & 08-SB5-12/13 SME & 10,005 & 44.7 & 34.4 & 6.6 & 3.2 & -51.5 & 6.27 & SRNL-STI-2009-697 & DWPF Simulated Melter Feed \\
\hline Citric Acid & 09-SB5-23 SME & 7,065 & 49.6 & 35.1 & 24.4 & 11.6 & -52.5 & 7.37 & SRNL-STI-2009-697 & DWPF Simulated Melter Feed \\
\hline Citric Acid & SB4-SME-1 & 4,000 & 41.2 & 33.7 & 3.05 & 1.39 & -54.4 & np & SRNL-STI-2011-674 & DWPF Simulated Melter Feed \\
\hline Citric Acid & SB4-SME-2 & 7,000 & 43.9 & 36 & 4.02 & 1.71 & -57.5 & np & SRNL-STI-2011-674 & DWPF Simulated Melter Feed \\
\hline Citric Acid & SB4-SME-1 & 7,000 & 41.2 & 33.7 & 3.05 & 1.22 & -60.0 & np & SRNL-STI-2011-674 & DWPF Simulated Melter Feed \\
\hline Citric Acid & SB4-SME-3 & 4,000 & 46.7 & 38.4 & 7.49 & 2.91 & -61.1 & np & SRNL-STI-2011-674 & DWPF Simulated Melter Feed \\
\hline Citric Acid & SB4-SME-2 & 10,000 & 43.9 & 36 & 4.02 & 1.56 & -61.2 & np & SRNL-STI-2011-674 & DWPF Simulated Melter Feed \\
\hline Citric Acid & SB4-SME-4 & 4,000 & 49.2 & 40.1 & 13.5 & 5.2 & -61.5 & np & SRNL-STI-2011-674 & DWPF Simulated Melter Feed \\
\hline Citric Acid & SB4-SME-5 & 4,000 & 51.9 & 42.5 & 23.8 & 8.9 & -62.6 & np & SRNL-STI-2011-674 & DWPF Simulated Melter Feed \\
\hline Citric Acid & SB4-SME-1 & 10,000 & 41.2 & 33.7 & 3.05 & 1.11 & -63.6 & np & SRNL-STI-2011-674 & DWPF Simulated Melter Feed \\
\hline Citric Acid & 09-SB5-23 SME & 10,055 & 49.6 & 35.1 & 24.4 & 8.5 & -65.2 & 7.37 & SRNL-STI-2009-697 & DWPF Simulated Melter Feed \\
\hline Citric Acid & SB4-SME-3 & 7,000 & 46.7 & 38.4 & 7.49 & 2.48 & -66.9 & np & SRNL-STI-2011-674 & DWPF Simulated Melter Feed \\
\hline Citric Acid & SB4-SME-3 & 10,000 & 46.7 & 38.4 & 7.49 & 2.4 & -68.0 & np & SRNL-STI-2011-674 & DWPF Simulated Melter Feed \\
\hline Citric Acid & SB4-SME-4 & 7,000 & 49.2 & 40.1 & 13.5 & 4.2 & -68.9 & $\mathrm{np}$ & SRNL-STI-2011-674 & DWPF Simulated Melter Feed \\
\hline Citric Acid & SB4-SME-4 & 10,000 & 49.2 & 40.1 & 13.5 & 4.1 & -69.6 & np & SRNL-STI-2011-674 & DWPF Simulated Melter Feed \\
\hline Citric Acid & SB4-SME-5 & 7,000 & 51.9 & 42.5 & 23.8 & 7.2 & -69.7 & np & SRNL-STI-2011-674 & DWPF Simulated Melter Feed \\
\hline Citric Acid & SB4-SME-5 & 10,000 & 51.9 & 42.5 & 23.8 & 6.7 & -71.8 & np & SRNL-STI-2011-674 & DWPF Simulated Melter Feed \\
\hline CTAB & AZ102 simulant & 1,000 & 12.6 & 12.1 & 4.6 & 7 & 52.2 & np & WSRC-MS-2003-136 & Hanford Simulated Waste \\
\hline Cytec P35 & FIU AZ-101 & 1,000 & np & $\mathrm{np}$ & 10.36 & 7.28 & -29.7 & np & SRNL-GPD-2004-40 & Hanford Simulated Waste \\
\hline Cytec P35 & AZ-101 & 1,000 & np & np & 3.22 & 2.94 & -8.7 & np & SRNL-GPD-2004-40 & Hanford Simulated Waste \\
\hline Cytec P70 & FIU AZ-101 & 1,000 & $\mathrm{np}$ & $\mathrm{np}$ & 10.36 & 8.83 & -14.8 & np & SRNL-GPD-2004-40 & Hanford Simulated Waste \\
\hline Cytec P70 & AZ-101 & 1,000 & np & np & 3.22 & 3.1 & -3.7 & np & SRNL-GPD-2004-40 & Hanford Simulated Waste \\
\hline Darvan C & AZ102 simulant & 1,000 & 12.6 & 12.1 & 4.6 & 4.4 & -4.3 & np & WSRC-MS-2003-136 & Hanford Simulated Waste \\
\hline Disperse-Ayd W-22 & AZ102 simulant & 1,000 & 12.6 & 12.1 & 4.6 & 4.6 & 0.0 & np & WSRC-MS-2003-136 & \begin{tabular}{|l} 
Hanford Simulated Waste \\
\end{tabular} \\
\hline Disperse-Ayd W-28 & AZ102 simulant & 1,000 & 12.6 & 12.1 & 4.6 & 4.9 & 6.5 & $\mathrm{np}$ & WSRC-MS-2003-136 & Hanford Simulated Waste \\
\hline Disperse-Ayd W-28 & SME & 460 & $\mathrm{np}$ & $\mathrm{np}$ & 10.5 & 10.8 & 2.9 & $\mathrm{np}$ & WSRC-TR-2004-82 & DWPF Simulated Melter Feed \\
\hline Disperse-Ayd W-28 & SRAT & 2,289 & np & np & 10.2 & 10.4 & 2.0 & np & WSRC-TR-2004-82 & $\begin{array}{l}\text { DWPF Simulated SRAT } \\
\text { Product }\end{array}$ \\
\hline Disperse-Ayd W-28 & SRAT & 1,147 & np & np & 10.2 & 9.6 & -5.9 & np & WSRC-TR-2004-82 & $\begin{array}{l}\text { DWPF Simulated SRAT } \\
\text { Product }\end{array}$ \\
\hline
\end{tabular}




\begin{tabular}{|c|c|c|c|c|c|c|c|c|c|c|}
\hline Modifier & Sample & $\begin{array}{l}\text { Mod conc, } \\
\text { ppm }\end{array}$ & $\begin{array}{c}\text { Total Solids, } \\
\%\end{array}$ & UDS, \% & \begin{tabular}{|c|} 
Yield \\
Stress \\
no Mod, \\
Pa \\
\end{tabular} & $\begin{array}{c}\text { Yield } \\
\text { Stress w } \\
\text { Mod, Pa }\end{array}$ & $\begin{array}{l}\text { Calc \% } \\
\text { Change }\end{array}$ & $\mathbf{p H}$ & Ref & Category \\
\hline Disperse-Ayd W-28 & SRAT & 460 & np & np & 10.2 & 9.5 & -6.9 & np & WSRC-TR-2004-82 & $\begin{array}{l}\text { DWPF Simulated SRAT } \\
\text { Product }\end{array}$ \\
\hline Disperse-Ayd W-28 & SME & 1,147 & np & np & 10.5 & 8.6 & -18.1 & np & WSRC-TR-2004-82 & DWPF Simulated Melter Feed \\
\hline Disperse-Ayd W-28 & SME & 2,289 & $\mathrm{np}$ & np & 10.5 & 6.1 & -41.9 & np & WSRC-TR-2004-82 & DWPF Simulated Melter Feed \\
\hline Disperse-Ayd W-28 & SRAT & 2,289 & np & np & 3.9 & 1.8 & -53.8 & np & WSRC-TR-2004-82 & $\begin{array}{l}\text { DWPF Simulated SRAT } \\
\text { Product }\end{array}$ \\
\hline Disperse-Ayd W-28 & SRAT & 4,554 & np & np & 3.9 & 1.5 & -61.5 & np & WSRC-TR-2004-82 & $\begin{array}{l}\text { DWPF Simulated SRAT } \\
\text { Product }\end{array}$ \\
\hline Disperse-Ayd W-28 & SRAT & 6,798 & np & np & 3.9 & 0.82 & -79.0 & np & WSRC-TR-2004-82 & $\begin{array}{l}\text { DWPF Simulated SRAT } \\
\text { Product }\end{array}$ \\
\hline Disperse-Ayd W-28 & SRAT & 9,020 & np & np & 3.9 & 0.6 & $\begin{array}{c}-84.6 \\
\end{array}$ & np & WSRC-TR-2004-82 & $\begin{array}{l}\text { DWPF Simulated SRAT } \\
\text { Product }\end{array}$ \\
\hline Dolapix A88 & 08-SB5-12/13 SME & 10,009 & 44.7 & 34.4 & 6.6 & 17 & 157.6 & 6.27 & SRNL-STI-2009-697 & DWPF Simulated Melter Feed \\
\hline Dolapix A88 & SB6-1,2,3,4 SME & 10,055 & 46.9 & 37.2 & 2.2 & 5.12 & 132.7 & 6.02 & SRNL-STI-2009-697 & DWPF Simulated Melter Feed \\
\hline Dolapix A88 & 08-SB5-12/13 SME & 6,132 & 44.7 & 34.4 & 6.6 & 13.9 & 110.6 & 6.27 & SRNL-STI-2009-697 & DWPF Simulated Melter Feed \\
\hline Dolapix A88 & SB6-1,2,3,4 SME & 6,149 & 46.9 & 37.2 & 2.2 & 4.25 & 93.2 & 6.02 & SRNL-STI-2009-697 & DWPF Simulated Melter Feed \\
\hline Dolapix A88 & 08-SB5-12/13 SME & 3,103 & 44.7 & 34.4 & 6.6 & 10.4 & 57.6 & 6.27 & SRNL-STI-2009-697 & DWPF Simulated Melter Feed \\
\hline Dolapix A88 & SB6-1,2,3,4 SME & 3,058 & 46.9 & 37.2 & 2.2 & 3.24 & 47.3 & 6.02 & SRNL-STI-2009-697 & DWPF Simulated Melter Feed \\
\hline Dolapix A88 & SB6-1,2,3,4 SME & 1,080 & 46.9 & 37.2 & 2.2 & 3.11 & 41.4 & 6.02 & SRNL-STI-2009-697 & DWPF Simulated Melter Feed \\
\hline Dolapix A88 & 08-SB5-12/13 SME & 1,057 & 44.7 & 34.4 & 6.6 & 8.7 & 31.8 & 6.27 & SRNL-STI-2009-697 & \begin{tabular}{|l|} 
DWPF Simulated Melter Feed \\
\end{tabular} \\
\hline Dolapix A88 & RuRhHg 1-9 SME & 9,838 & 51.55 & 42 & 13.4 & 16.4 & 22.4 & 4.7 & SRNL-STI-2009-697 & \begin{tabular}{|l|} 
DWPF Simulated Melter Feed \\
\end{tabular} \\
\hline Dolapix A88 & RuRhHg 1-9 SME & 847 & 51.55 & 42 & 13.4 & 12.9 & -3.7 & 4.7 & SRNL-STI-2009-697 & DWPF Simulated Melter Feed \\
\hline Dolapix A88 & RuRhHg 1-9 SME & 2,902 & 51.55 & 42 & 13.4 & 12 & -10.4 & 4.7 & SRNL-STI-2009-697 & DWPF Simulated Melter Feed \\
\hline Dolapix A88 & RuRhHg 1-9 SME & 5,889 & 51.55 & 42 & 13.4 & 11.9 & -11.2 & 4.7 & SRNL-STI-2009-697 & DWPF Simulated Melter Feed \\
\hline Dolapix A88 & Melter Feed & 1,000 & 47 & 41 & 10.3 & 12.9 & 25.2 & $6-7$ & WSRC-MS-2003-136 & DWPF Simulated Melter Feed \\
\hline Dolapix CE64 & SB6-1,2,3,4 SME & 6,138 & 46.9 & 37.2 & 2.2 & 3.36 & 52.7 & 6.02 & SRNL-STI-2009-697 & DWPF Simulated Melter Feed \\
\hline Dolapix CE64 & SB6-1,2,3,4 SME & 11,126 & 46.9 & 37.2 & 2.2 & 3.23 & 46.8 & 6.02 & SRNL-STI-2009-697 & DWPF Simulated Melter Feed \\
\hline Dolapix CE64 & 08-SB5-12/13 SME & 11,009 & 44.7 & 34.4 & 6.6 & 9.5 & 43.9 & 6.27 & SRNL-STI-2009-697 & DWPF Simulated Melter Feed \\
\hline Dolapix CE64 & 08-SB5-12/13 SME & 6,074 & 44.7 & 34.4 & 6.6 & 9 & 36.4 & 6.27 & SRNL-STI-2009-697 & DWPF Simulated Melter Feed \\
\hline Dolapix CE64 & SB6-1,2,3,4 SME & 1,044 & 46.9 & 37.2 & 2.2 & 2.91 & 32.3 & 6.02 & SRNL-STI-2009-697 & DWPF Simulated Melter Feed \\
\hline Dolapix CE64 & 08-SB5-12/13 SME & 16,057 & 44.7 & 34.4 & 6.6 & 8.5 & 28.8 & 6.27 & SRNL-STI-2009-697 & DWPF Simulated Melter Feed \\
\hline Dolapix CE64 & SB6-1,2,3,4 SME & 16,049 & 46.9 & 37.2 & 2.2 & 2.65 & 20.5 & 6.02 & SRNL-STI-2009-697 & DWPF Simulated Melter Feed \\
\hline Dolapix CE64 & 08-SB5-12/13 SME & 1,058 & 44.7 & 34.4 & 6.6 & 7.2 & 9.1 & 6.27 & SRNL-STI-2009-697 & DWPF Simulated Melter Feed \\
\hline Dolapix CE64 & SME & 1,621 & np & np & 15.8 & 16.4 & 3.8 & np & WSRC-TR-2004-82 & DWPF Simulated Melter Feed \\
\hline Dolapix CE64 & SRAT & 649 & np & np & 10.2 & 10.4 & 2.0 & np & WSRC-TR-2004-82 & $\begin{array}{l}\text { DWPF Simulated SRAT } \\
\text { Product }\end{array}$ \\
\hline Dolapix CE64 & RuRhHg 1-9 SME & 946 & 51.55 & 42 & 13.4 & 12.5 & -6.7 & 4.7 & SRNL-STI-2009-697 & DWPF Simulated Melter Feed \\
\hline Dolapix CE64 & SME & 649 & $\mathrm{np}$ & $\mathrm{np}$ & 10.5 & 9.7 & -7.6 & $\mathrm{np}$ & WSRC-TR-2004-82 & DWPF Simulated Melter Feed \\
\hline Dolapix CE64 & SME & 1,621 & $\mathrm{np}$ & $\mathrm{np}$ & 10.5 & 9.66 & -8.0 & $\mathrm{np}$ & WSRC-TR-2004-82 & DWPF Simulated Melter Feed \\
\hline
\end{tabular}




\begin{tabular}{|c|c|c|c|c|c|c|c|c|c|c|}
\hline Modifier & Sample & $\begin{array}{l}\text { Mod conc, } \\
\text { ppm }\end{array}$ & $\begin{array}{c}\text { Total Solids, } \\
\quad \%\end{array}$ & UDS, \% & \begin{tabular}{|c|} 
Yield \\
Stress \\
no Mod, \\
Pa \\
\end{tabular} & $\begin{array}{c}\text { Yield } \\
\text { Stress w } \\
\text { Mod, Pa }\end{array}$ & $\begin{array}{l}\text { Calc \% } \\
\text { Change }\end{array}$ & pH & Ref & Category \\
\hline Dolapix CE64 & SRAT & 747 & np & np & 11.8 & 10.8 & -8.5 & np & WSRC-TR-2004-82 & $\begin{array}{l}\text { DWPF Simulated SRAT } \\
\text { Product }\end{array}$ \\
\hline Dolapix CE64 & $\begin{array}{l}\text { RuRhHg 1-9 Decanted } \\
\text { SME }\end{array}$ & 1,095 & np & np & 72.5 & 65.4 & -9.8 & 4.59 & SRNL-STI-2009-697 & DWPF Simulated Melter Feed \\
\hline Dolapix CE64 & SRAT & 1,621 & np & np & 10.2 & 9.2 & -9.8 & np & WSRC-TR-2004-82 & $\begin{array}{l}\text { DWPF Simulated SRAT } \\
\text { Product }\end{array}$ \\
\hline Dolapix CE64 & SME & 3,234 & $\mathrm{np}$ & $\mathrm{np}$ & 15.8 & 13.8 & -12.7 & $\mathrm{np}$ & WSRC-TR-2004-82 & DWPF Simulated Melter Feed \\
\hline Dolapix CE64 & SRAT & 1,492 & np & np & 11.8 & 10.2 & $\begin{array}{c}-13.6 \\
\end{array}$ & $\mathrm{np}$ & WSRC-TR-2004-82 & $\begin{array}{l}\text { DWPF Simulated SRAT } \\
\text { Product }\end{array}$ \\
\hline \begin{tabular}{|l|} 
Dolapix CE64 \\
\end{tabular} & SME & 3,234 & np & np & 10.5 & 8.24 & -21.5 & $\mathrm{np}$ & WSRC-TR-2004-82 & DWPF Simulated Melter Feed \\
\hline Dolapix CE64 & SRAT & 3,234 & np & np & 10.2 & 8 & -21.6 & np & WSRC-TR-2004-82 & $\begin{array}{l}\text { DWPF Simulated SRAT } \\
\text { Product }\end{array}$ \\
\hline \begin{tabular}{|l} 
Dolapix CE64 \\
\end{tabular} & RuRhHg 1-9 SME & 5,962 & 51.55 & 42 & 13.4 & 10.2 & -23.9 & 4.7 & SRNL-STI-2009-697 & DWPF Simulated Melter Feed \\
\hline Dolapix CE64 & $\begin{array}{l}\text { RuRhHg 1-9 Decanted } \\
\text { SME }\end{array}$ & 6,015 & np & np & 72.5 & 50.5 & -30.3 & 4.59 & SRNL-STI-2009-697 & DWPF Simulated Melter Feed \\
\hline \begin{tabular}{|l|} 
Dolapix CE64 \\
\end{tabular} & AZ-101 & 1,000 & np & np & 3.22 & 2.22 & -31.1 & np & SRNL-GPD-2004-40 & Hanford Simulated Waste \\
\hline Dolapix CE64 & FIU AZ-101 & 1,000 & np & np & 10.36 & 7.01 & -32.3 & np & SRNL-GPD-2004-40 & Hanford Simulated Waste \\
\hline Dolapix CE64 & SRAT & 3,234 & np & np & 3.9 & 2.5 & -35.9 & np & WSRC-TR-2004-82 & $\begin{array}{l}\text { DWPF Simulated SRAT } \\
\text { Product }\end{array}$ \\
\hline Dolapix CE64 & RuRhHg 1-9 SME & 10,928 & 51.55 & 42 & 13.4 & 8.5 & -36.6 & 4.7 & SRNL-STI-2009-697 & DWPF Simulated Melter Feed \\
\hline Dolapix CE64 & SRAT & 6,436 & np & np & 3.9 & 2.3 & -41.0 & np & WSRC-TR-2004-82 & $\begin{array}{l}\text { DWPF Simulated SRAT } \\
\text { Product }\end{array}$ \\
\hline Dolapix CE64 & $\begin{array}{l}\text { RuRhHg 1-9 Decanted } \\
\text { SME }\end{array}$ & 11,001 & np & np & 72.5 & 42.2 & -41.8 & 4.59 & SRNL-STI-2009-697 & DWPF Simulated Melter Feed \\
\hline \begin{tabular}{|l} 
Dolapix CE64 \\
\end{tabular} & RuRhHg 1-9 SME & 15,974 & 51.55 & 42 & 13.4 & 7.6 & -43.3 & 4.7 & SRNL-STI-2009-697 & DWPF Simulated Melter Feed \\
\hline \begin{tabular}{|l|} 
Dolapix CE64 \\
\end{tabular} & SRAT & 9,606 & np & np & 3.9 & 2.1 & -46.2 & np & WSRC-TR-2004-82 & $\begin{array}{l}\text { DWPF Simulated SRAT } \\
\text { Product }\end{array}$ \\
\hline Dolapix CE64 & $\begin{array}{l}\text { RuRhHg 1-9 Decanted } \\
\text { SME }\end{array}$ & 16,051 & np & np & 72.5 & 37.2 & -48.7 & 4.59 & SRNL-STI-2009-697 & DWPF Simulated Melter Feed \\
\hline Dolapix CE64 & SRAT & 12,745 & np & np & 3.9 & 1.7 & -56.4 & np & WSRC-TR-2004-82 & $\begin{array}{l}\text { DWPF Simulated SRAT } \\
\text { Product }\end{array}$ \\
\hline \begin{tabular}{|l|} 
Dolapix PC75 \\
\end{tabular} & 08-SB5-12/13 SME & 16,055 & 44.7 & 34.4 & 6.6 & 6.6 & 0.0 & 6.27 & SRNL-STI-2009-697 & DWPF Simulated Melter Feed \\
\hline \begin{tabular}{|l|} 
Dolapix PC75 \\
\end{tabular} & 08-SB5-12/13 SME & 1,034 & 44.7 & 34.4 & 6.6 & 6.4 & -3.0 & 6.27 & SRNL-STI-2009-697 & DWPF Simulated Melter Feed \\
\hline \begin{tabular}{|l|} 
Dolapix PC75 \\
\end{tabular} & SB6-1,2,3,4 SME & 1,083 & 46.9 & 37.2 & 2.2 & 2.12 & -3.6 & 6.02 & SRNL-STI-2009-697 & DWPF Simulated Melter Feed \\
\hline \begin{tabular}{|l} 
Dolapix PC75 \\
\end{tabular} & 08-SB5-12/13 SME & 11,083 & 44.7 & 34.4 & 6.6 & 6.3 & -4.5 & 6.27 & SRNL-STI-2009-697 & DWPF Simulated Melter Feed \\
\hline \begin{tabular}{|l} 
Dolapix PC75 \\
\end{tabular} & 08-SB5-12/13 SME & 6,096 & 44.7 & 34.4 & 6.6 & 5.9 & -10.6 & 6.27 & SRNL-STI-2009-697 & DWPF Simulated Melter Feed \\
\hline Dolapix PC75 & SB6-1,2,3,4 SME & 16,519 & 46.9 & 37.2 & 2.2 & 1.88 & -14.5 & 6.02 & SRNL-STI-2009-697 & DWPF Simulated Melter Feed \\
\hline Dolapix PC75 & SB6-1,2,3,4 SME & 10,998 & 46.9 & 37.2 & 2.2 & 1.84 & -16.4 & 6.02 & SRNL-STI-2009-697 & DWPF Simulated Melter Feed \\
\hline \begin{tabular}{|l} 
Dolapix PC75 \\
\end{tabular} & RuRhHg 1-9 SME & 984 & 51.55 & 42 & 13.4 & 11.1 & -17.2 & 4.7 & SRNL-STI-2009-697 & DWPF Simulated Melter Feed \\
\hline Dolapix PC75 & SB6-1,2,3,4 SME & 6,084 & 46.9 & 37.2 & 2.2 & 1.78 & -19.1 & 6.02 & SRNL-STI-2009-697 & DWPF Simulated Melter Feed \\
\hline
\end{tabular}




\begin{tabular}{|c|c|c|c|c|c|c|c|c|c|c|}
\hline Modifier & Sample & $\begin{array}{c}\text { Mod conc, } \\
\text { ppm }\end{array}$ & $\begin{array}{c}\text { Total Solids, } \\
\%\end{array}$ & UDS, \% & \begin{tabular}{|c|} 
Yield \\
Stress \\
no Mod, \\
Pa \\
\end{tabular} & $\begin{array}{c}\text { Yield } \\
\text { Stress w } \\
\text { Mod, Pa }\end{array}$ & $\begin{array}{l}\text { Calc \% } \\
\text { Change }\end{array}$ & pH & Ref & Category \\
\hline Dolapix PC75 & RuRhHg 1-9 SME & 5,987 & 51.55 & 42 & 13.4 & 10 & -25.4 & 4.7 & SRNL-STI-2009-697 & DWPF Simulated Melter Feed \\
\hline Dolapix PC75 & RuRhHg 1-9 SME & 11,001 & 51.55 & 42 & 13.4 & 9.2 & -31.3 & 4.7 & SRNL-STI-2009-697 & DWPF Simulated Melter Feed \\
\hline Dolapix PC75 & RuRhHg 1-9 SME & 15,990 & 51.55 & 42 & 13.4 & 9.1 & -32.1 & 4.7 & SRNL-STI-2009-697 & DWPF Simulated Melter Feed \\
\hline Duramax 3005 & AZ102 simulant & 1,000 & 47 & 41 & 4.6 & 3.8 & -17.4 & np & WSRC-MS-2003-136 & Hanford Simulated Waste \\
\hline Duramax 3005 & FIU AZ-101 & 1,000 & np & np & 10.36 & 7.49 & -27.7 & 9.85 & SRNL-GPD-2004-40 & Hanford Simulated Waste \\
\hline Duramax 3005 & AZ-101 & 1,000 & np & np & 3.22 & 2.84 & -11.8 & 12.23 & SRNL-GPD-2004-40 & Hanford Simulated Waste \\
\hline Duramax D3005 & Melter Feed & 1,000 & 47 & 41 & 10.3 & 14.6 & 41.7 & 6-7 & WSRC-MS-2003-136 & DWPF Simulated Melter Feed \\
\hline EDAPLAN 470 & AZ-101 & 1,000 & $\mathrm{np}$ & np & 3.22 & 3.19 & -0.9 & $\mathrm{np}$ & SRNL-GPD-2004-40 & Hanford Simulated Waste \\
\hline EDAPLAN 470 & FIU AZ-101 & 1,000 & np & np & 10.36 & 9.41 & -9.2 & $\mathrm{np}$ & SRNL-GPD-2004-40 & Hanford Simulated Waste \\
\hline EDAPLAN 472 & AZ-101 & 1,000 & $\mathrm{np}$ & np & 3.22 & 3.34 & 3.7 & $\mathrm{np}$ & SRNL-GPD-2004-40 & Hanford Simulated Waste \\
\hline EDAPLAN 472 & FIU AZ-101 & 1,000 & np & np & 10.36 & 9.42 & -9.1 & 9.85 & SRNL-GPD-2004-40 & Hanford Simulated Waste \\
\hline Glycolic Acid & SB6 SME-1 & 1,000 & 28.2 & 20.2 & 4.25 & 4.86 & 14.4 & $\mathrm{np}$ & SRNL-STI-2011-674 & DWPF Simulated Melter Feed \\
\hline Glycolic Acid & SB6 SME-1 & 5,500 & 28.2 & 20.2 & 4.25 & 4.76 & 12.0 & np & SRNL-STI-2011-674 & DWPF Simulated Melter Feed \\
\hline Glycolic Acid & SB6 SME-1 & 9,500 & 28.2 & 20.2 & 4.25 & 4.73 & 11.3 & np & SRNL-STI-2011-674 & DWPF Simulated Melter Feed \\
\hline \begin{tabular}{||l|} 
Glycolic Acid \\
\end{tabular} & SB6 SME-2 & 1,000 & 30.9 & 22.3 & 5.98 & 6.49 & 8.5 & np & SRNL-STI-2011-674 & DWPF Simulated Melter Feed \\
\hline \begin{tabular}{|l|l} 
Glycolic Acid \\
\end{tabular} & SB6 SME-1 & 14,000 & 28.2 & 20.2 & 4.25 & 4.5 & 5.9 & $\mathrm{np}$ & SRNL-STI-2011-674 & DWPF Simulated Melter Feed \\
\hline Glycolic Acid & SB6 SME-2 & 14,000 & 30.9 & 22.3 & 5.98 & 6.22 & 4.0 & $\mathrm{np}$ & SRNL-STI-2011-674 & DWPF Simulated Melter Feed \\
\hline Glycolic Acid & SB6 SME-2 & 9,500 & 30.9 & 22.3 & 5.98 & 6.21 & 3.8 & np & SRNL-STI-2011-674 & DWPF Simulated Melter Feed \\
\hline Glycolic Acid & SB6 SME-3 & 1,000 & 33 & 23.8 & 7.82 & 8.06 & 3.1 & np & SRNL-STI-2011-674 & DWPF Simulated Melter Feed \\
\hline Glycolic Acid & SB6 SME-8 & 1,000 & 46.4 & 34.9 & 26.4 & 27 & 2.3 & np & SRNL-STI-2011-674 & DWPF Simulated Melter Feed \\
\hline Glycolic Acid & SB6 SME-4 & 1,000 & 36 & 26.4 & 10.8 & 11 & 1.9 & np & SRNL-STI-2011-674 & DWPF Simulated Melter Feed \\
\hline \begin{tabular}{|l|} 
Glycolic Acid \\
\end{tabular} & SB6 SME-2 & 5,500 & 30.9 & 22.3 & 5.98 & 6.08 & 1.7 & np & SRNL-STI-2011-674 & DWPF Simulated Melter Feed \\
\hline Glycolic Acid & SB6 SME-9 & 1,000 & 48.2 & 36.4 & 31.2 & 31.7 & 1.6 & $\mathrm{np}$ & SRNL-STI-2011-674 & DWPF Simulated Melter Feed \\
\hline \begin{tabular}{||l} 
Glycolic Acid \\
\end{tabular} & SB6 SME-7 & 1,000 & 43.6 & 32.4 & 24.9 & 25.1 & 0.8 & np & SRNL-STI-2011-674 & DWPF Simulated Melter Feed \\
\hline Glycolic Acid & SB6 SME-9 & 5,500 & 48.2 & 36.4 & 31.2 & 31.3 & 0.3 & np & SRNL-STI-2011-674 & DWPF Simulated Melter Feed \\
\hline Glycolic Acid & SB6 SME-4 & 9,500 & 36 & 26.4 & 10.8 & 10.8 & 0.0 & np & SRNL-STI-2011-674 & DWPF Simulated Melter Feed \\
\hline Glycolic Acid & SB6 SME-4 & 5,500 & 36 & 26.4 & 10.8 & 10.7 & -0.9 & np & SRNL-STI-2011-674 & DWPF Simulated Melter Feed \\
\hline Glycolic Acid & SB6 SME-6 & 9,500 & 41.5 & 30.8 & 19 & 18.8 & -1.1 & np & SRNL-STI-2011-674 & DWPF Simulated Melter Feed \\
\hline Glycolic Acid & SB6 SME-3 & 9,500 & 33 & 23.8 & 7.82 & 7.71 & -1.4 & $\mathrm{np}$ & SRNL-STI-2011-674 & DWPF Simulated Melter Feed \\
\hline Glycolic Acid & SB6 SME-6 & 1,000 & 41.5 & 30.8 & 19 & 18.7 & -1.6 & np & SRNL-STI-2011-674 & DWPF Simulated Melter Feed \\
\hline Glycolic Acid & SB6 SME-4 & 14,000 & 36 & 26.4 & 10.8 & 10.6 & -1.9 & np & SRNL-STI-2011-674 & DWPF Simulated Melter Feed \\
\hline Glycolic Acid & SB6 SME-3 & 5,500 & 33 & 23.8 & 7.82 & 7.64 & -2.3 & np & SRNL-STI-2011-674 & DWPF Simulated Melter Feed \\
\hline Glycolic Acid & SB6 SME-3 & 14,000 & 33 & 23.8 & 7.82 & 7.64 & -2.3 & np & SRNL-STI-2011-674 & DWPF Simulated Melter Feed \\
\hline Glycolic Acid & SB6 SME-6 & 5,500 & 41.5 & 30.8 & 19 & 18.4 & -3.2 & np & SRNL-STI-2011-674 & DWPF Simulated Melter Feed \\
\hline Glycolic Acid & SB6 SME-8 & 9,500 & 46.4 & 34.9 & 26.4 & 25.4 & -3.8 & np & SRNL-STI-2011-674 & DWPF Simulated Melter Feed \\
\hline Glycolic Acid & SB6 SME-9 & 9,500 & 48.2 & 36.4 & 31.2 & 30 & -3.8 & np & SRNL-STI-2011-674 & DWPF Simulated Melter Feed \\
\hline Glycolic Acid & SB6 SME-5 & 9,500 & 38.6 & 28.3 & 14.5 & 13.9 & -4.1 & np & SRNL-STI-2011-674 & DWPF Simulated Melter Feed \\
\hline \begin{tabular}{|l|l} 
Glycolic Acid \\
\end{tabular} & SB6 SME-8 & 5,500 & 46.4 & 34.9 & 26.4 & 25.1 & -4.9 & $\mathrm{np}$ & SRNL-STI-2011-674 & DWPF Simulated Melter Feed \\
\hline Glycolic Acid & SB6 SME-5 & 1,000 & 38.6 & 28.3 & 14.5 & 13.7 & -5.5 & np & SRNL-STI-2011-674 & DWPF Simulated Melter Feed \\
\hline
\end{tabular}




\begin{tabular}{|c|c|c|c|c|c|c|c|c|c|c|}
\hline Modifier & Sample & $\begin{array}{l}\text { Mod conc, } \\
\text { ppm }\end{array}$ & $\begin{array}{c}\text { Total Solids, } \\
\%\end{array}$ & UDS, \% & \begin{tabular}{|c|} 
Yield \\
Stress \\
no Mod, \\
Pa \\
\end{tabular} & $\begin{array}{c}\text { Yield } \\
\text { Stress w } \\
\text { Mod, Pa }\end{array}$ & $\begin{array}{l}\text { Calc \% } \\
\text { Change }\end{array}$ & $\mathbf{p H}$ & Ref & Category \\
\hline Glycolic Acid & SB6 SME-7 & 5,500 & 43.6 & 32.4 & 24.9 & 23.4 & -6.0 & np & SRNL-STI-2011-674 & \begin{tabular}{|l|} 
DWF Simulated Melter Feed \\
\end{tabular} \\
\hline Glycolic Acid & SB6 SME-8 & 14,000 & 46.4 & 34.9 & 26.4 & 24.8 & -6.1 & np & SRNL-STI-2011-674 & DWPF Simulated Melter Feed \\
\hline Glycolic Acid & SB6 SME-7 & 14,000 & 43.6 & 32.4 & 24.9 & 23.2 & -6.8 & $\mathrm{np}$ & SRNL-STI-2011-674 & DWPF Simulated Melter Feed \\
\hline Glycolic Acid & SB6 SME-6 & 14,000 & 41.5 & 30.8 & 19 & 17.7 & -6.8 & np & SRNL-STI-2011-674 & DWPF Simulated Melter Feed \\
\hline Glycolic Acid & SB6 SME-5 & 5,500 & 38.6 & 28.3 & 14.5 & 13.5 & -6.9 & $\mathrm{np}$ & SRNL-STI-2011-674 & DWPF Simulated Melter Feed \\
\hline Glycolic Acid & SB6 SME-5 & 14,000 & 38.6 & 28.3 & 14.5 & 13.3 & -8.3 & np & SRNL-STI-2011-674 & DWPF Simulated Melter Feed \\
\hline Glycolic Acid & SB6 SME-9 & 14,000 & 48.2 & 36.4 & 31.2 & 28.6 & -8.3 & np & SRNL-STI-2011-674 & DWPF Simulated Melter Feed \\
\hline Glycolic Acid & SB6 SME-7 & 9,500 & 43.6 & 32.4 & 24.9 & 22.6 & -9.2 & np & SRNL-STI-2011-674 & DWPF Simulated Melter Feed \\
\hline Glycolic Acid Solution & SB4-SME-2 & 1,000 & 43.9 & 36 & 4.02 & 3.98 & -1.0 & np & SRNL-STI-2011-674 & DWPF Simulated Melter Feed \\
\hline Glycolic Acid Solution & SB4-SME-4 & 1,000 & 49.2 & 40.1 & 13.5 & 12.5 & -7.4 & np & SRNL-STI-2011-674 & \begin{tabular}{|l} 
DWPF Simulated Melter Feed \\
\end{tabular} \\
\hline Glycolic Acid Solution & SB4-SME-3 & 1,000 & 46.7 & 38.4 & 7.49 & 6.69 & -10.7 & $\mathrm{np}$ & SRNL-STI-2011-674 & DWPF Simulated Melter Feed \\
\hline Glycolic Acid Solution & SB4-SME-1 & 1,000 & 41.2 & 33.7 & 3.05 & 2.67 & -12.5 & $\mathrm{np}$ & SRNL-STI-2011-674 & DWPF Simulated Melter Feed \\
\hline Glycolic Acid Solution & SB4-SME-2 & 5,500 & 43.9 & 36 & 4.02 & 3.06 & -23.9 & np & SRNL-STI-2011-674 & DWPF Simulated Melter Feed \\
\hline Glycolic Acid Solution & SB4-SME-2 & 9,500 & 43.9 & 36 & 4.02 & 2.57 & -36.1 & np & SRNL-STI-2011-674 & DWPF Simulated Melter Feed \\
\hline Glycolic Acid Solution & SB4-SME-3 & 5,500 & 46.7 & 38.4 & 7.49 & 4.69 & -37.4 & np & SRNL-STI-2011-674 & DWPF Simulated Melter Feed \\
\hline Glycolic Acid Solution & SB4-SME-4 & 5,500 & 49.2 & 40.1 & 13.5 & 8.3 & -38.5 & $\mathrm{np}$ & SRNL-STI-2011-674 & DWPF Simulated Melter Feed \\
\hline Glycolic Acid Solution & SB4-SME-2 & 14,000 & 43.9 & 36 & 4.02 & 2.45 & -39.1 & $\mathrm{np}$ & SRNL-STI-2011-674 & DWPF Simulated Melter Feed \\
\hline Glycolic Acid Solution & SB4-SME-1 & 9,500 & 41.2 & 33.7 & 3.05 & 1.85 & -39.3 & np & SRNL-STI-2011-674 & DWPF Simulated Melter Feed \\
\hline Glycolic Acid Solution & SB4-SME-3 & 9,500 & 46.7 & 38.4 & 7.49 & 4.23 & -43.5 & np & SRNL-STI-2011-674 & DWPF Simulated Melter Feed \\
\hline Glycolic Acid Solution & SB4-SME-1 & 5,500 & 41.2 & 33.7 & 3.05 & 1.72 & -43.6 & np & SRNL-STI-2011-674 & \begin{tabular}{|l|} 
DWPF Simulated Melter Feed \\
\end{tabular} \\
\hline Glycolic Acid Solution & SB4-SME-1 & 14,000 & 41.2 & 33.7 & 3.05 & 1.69 & -44.6 & np & SRNL-STI-2011-674 & \begin{tabular}{|l|} 
DWPF Simulated Melter Feed \\
\end{tabular} \\
\hline Glycolic Acid Solution & SB4-SME-3 & 14,000 & 46.7 & 38.4 & 7.49 & 3.67 & -51.0 & $\mathrm{np}$ & SRNL-STI-2011-674 & \begin{tabular}{|l|} 
DWPF Simulated Melter Feed \\
\end{tabular} \\
\hline Glycolic Acid Solution & SB4-SME-4 & 9,500 & 49.2 & 40.1 & 13.5 & 6.5 & -51.9 & $\mathrm{np}$ & SRNL-STI-2011-674 & DWPF Simulated Melter Feed \\
\hline Glycolic Acid Solution & SB4-SME-4 & 14,000 & 49.2 & 40.1 & 13.5 & 6.3 & -53.3 & np & SRNL-STI-2011-674 & DWPF Simulated Melter Feed \\
\hline Phenylboric Acid & $\begin{array}{l}\text { RuRhHg 1-9 Decanted } \\
\text { SME }\end{array}$ & 1,045 & np & np & 72.5 & 66.8 & -7.9 & 4.59 & SRNL-STI-2009-697 & DWPF Simulated Melter Feed \\
\hline Phenylboric Acid & $\begin{array}{l}\text { RuRhHg 1-9 Decanted } \\
\text { SME }\end{array}$ & 3,506 & np & np & 72.5 & 66.3 & -8.6 & 4.59 & SRNL-STI-2009-697 & DWPF Simulated Melter Feed \\
\hline Phenylboric Acid & $\begin{array}{l}\text { RuRhHg 1-9 Decanted } \\
\text { SME }\end{array}$ & 7,033 & np & np & 72.5 & 68.4 & -5.7 & 4.59 & SRNL-STI-2009-697 & DWPF Simulated Melter Feed \\
\hline Phenylboric Acid & $\begin{array}{l}\text { RuRhHg 1-9 Decanted } \\
\text { SME }\end{array}$ & 10,018 & np & np & 72.5 & 66.1 & -8.8 & 4.59 & SRNL-STI-2009-697 & DWPF Simulated Melter Feed \\
\hline Polyacrylic Acid & SB6-1,2,3,4 SME & 1,025 & 46.9 & 37.2 & 2.2 & 3.25 & 47.7 & 6.02 & SRNL-STI-2009-697 & \begin{tabular}{|l|} 
DWPF Simulated Melter Feed \\
\end{tabular} \\
\hline Polyacrylic Acid & SB6-1,2,3,4 SME & 3,589 & 46.9 & 37.2 & 2.2 & 2.96 & 34.5 & 6.02 & SRNL-STI-2009-697 & DWPF Simulated Melter Feed \\
\hline Polyacrylic Acid & 08-SB5-12/13 SME & 3,575 & 44.7 & 34.4 & 6.6 & 8.7 & 31.8 & 6.27 & SRNL-STI-2009-697 & DWPF Simulated Melter Feed \\
\hline Polyacrylic Acid & 09-SB5-24 SME & 1,020 & 48.8 & 34.5 & 5.22 & 6.45 & 23.6 & np & SRNL-STI-2009-697 & DWPF Simulated Melter Feed \\
\hline Polyacrylic Acid & 09-SB5-24 SME & 3,528 & 48.8 & 34.5 & 5.22 & 6.08 & 16.5 & np & SRNL-STI-2009-697 & DWPF Simulated Melter Feed \\
\hline Polyacrylic Acid & 08-SB5-12/13 SME & 976 & 44.7 & 34.4 & 6.6 & 7.6 & 15.2 & 6.27 & SRNL-STI-2009-697 & DWPF Simulated Melter Feed \\
\hline Polyacrylic Acid & SB6-1,2,3,4 SME & 7,010 & 46.9 & 37.2 & 2.2 & 2.38 & 8.2 & 6.02 & SRNL-STI-2009-697 & DWPF Simulated Melter Feed \\
\hline
\end{tabular}




\begin{tabular}{|c|c|c|c|c|c|c|c|c|c|c|}
\hline Modifier & Sample & $\begin{array}{l}\text { Mod conc, } \\
\text { ppm }\end{array}$ & $\begin{array}{c}\text { Total Solids, } \\
\%\end{array}$ & UDS, \% & $\begin{array}{c}\text { Yield } \\
\text { Stress } \\
\text { no Mod, } \\
\text { Pa } \\
\end{array}$ & $\begin{array}{c}\text { Yield } \\
\text { Stress w } \\
\text { Mod, Pa }\end{array}$ & $\begin{array}{l}\text { Calc \% } \\
\text { Change }\end{array}$ & $\mathbf{p H}$ & Ref & Category \\
\hline Polyacrylic Acid & 09-SB5-23 SME & 988 & 49.6 & 35.1 & 24.4 & 24.8 & 1.6 & 7.37 & SRNL-STI-2009-697 & DWPF Simulated Melter Feed \\
\hline Polyacrylic Acid & 08-SB5-12/13 SME & 7,166 & 44.7 & 34.4 & 6.6 & 6.5 & -1.5 & 6.27 & SRNL-STI-2009-697 & DWPF Simulated Melter Feed \\
\hline Polyacrylic Acid & 09-SB5-23 SME & 3,548 & 49.6 & 35.1 & 24.4 & 20.6 & -15.6 & 7.37 & SRNL-STI-2009-697 & \begin{tabular}{|l|} 
DWPF Simulated Melter Feed \\
\end{tabular} \\
\hline Polyacrylic Acid & 09-SB5-24 SME & 7,096 & 48.8 & 34.5 & 5.22 & 4.12 & -21.1 & np & SRNL-STI-2009-697 & DWPF Simulated Melter Feed \\
\hline Polyacrylic Acid & SB6-1,2,3,4 SME & 9,996 & 46.9 & 37.2 & 2.2 & 1.61 & -26.8 & 6.02 & SRNL-STI-2009-697 & DWPF Simulated Melter Feed \\
\hline Polyacrylic Acid & RuRhHg 1-9 SME & 937 & 51.55 & 42 & 13.4 & 8.4 & -37.3 & 4.7 & SRNL-STI-2009-697 & DWPF Simulated Melter Feed \\
\hline Polyacrylic Acid & RuRhHg 1-9 SME & 9,954 & 51.55 & 42 & 13.4 & 8.1 & -39.6 & 4.7 & SRNL-STI-2009-697 & DWPF Simulated Melter Feed \\
\hline Polyacrylic Acid & \begin{tabular}{|l} 
RuRhHg 1-9 Decanted \\
SME
\end{tabular} & 992 & np & np & 72.5 & 39.5 & -45.5 & 4.59 & SRNL-STI-2009-697 & DWPF Simulated Melter Feed \\
\hline Polyacrylic Acid & RuRhHg 1-9 SME & 6,930 & 51.55 & 42 & 13.4 & 6.9 & -48.5 & 4.7 & SRNL-STI-2009-697 & DWPF Simulated Melter Feed \\
\hline Polyacrylic Acid & 09-SB5-23 SME & 6,986 & 49.6 & 35.1 & 24.4 & 12.5 & -48.8 & 7.37 & SRNL-STI-2009-697 & DWPF Simulated Melter Feed \\
\hline Polyacrylic Acid & 09-SB5-24 SME & 10,158 & 48.8 & 34.5 & 5.22 & 2.67 & $\begin{array}{l}-48.9 \\
\end{array}$ & np & SRNL-STI-2009-697 & DWPF Simulated Melter Feed \\
\hline Polyacrylic Acid & RuRhHg 1-9 SME & 3,466 & 51.55 & 42 & 13.4 & 6.8 & -49.3 & 4.7 & SRNL-STI-2009-697 & DWPF Simulated Melter Feed \\
\hline Polyacrylic Acid & 08-SB5-12/13 SME & 10,509 & 44.7 & 34.4 & 6.6 & 3.3 & -50.0 & 6.27 & SRNL-STI-2009-697 & DWPF Simulated Melter Feed \\
\hline Polyacrylic Acid & \begin{tabular}{|l} 
RuRhHg 1-9 Decanted \\
SME
\end{tabular} & 10,003 & np & np & 72.5 & 26.7 & -63.2 & 4.59 & SRNL-STI-2009-697 & DWPF Simulated Melter Feed \\
\hline Polyacrylic Acid & $\begin{array}{l}\text { RuRhHg 1-9 Decanted } \\
\text { SME }\end{array}$ & 3,491 & np & np & 72.5 & 25.2 & -65.2 & 4.59 & SRNL-STI-2009-697 & DWPF Simulated Melter Feed \\
\hline Polyacrylic Acid & $\begin{array}{l}\begin{array}{l}\text { RuRhHg 1-9 Decanted } \\
\text { SME }\end{array} \\
\end{array}$ & 7,009 & np & np & 72.5 & 23.1 & -68.1 & 4.59 & SRNL-STI-2009-697 & DWPF Simulated Melter Feed \\
\hline Polyacrylic Acid & 09-SB5-23 SME & 10,010 & 49.6 & 35.1 & 24.4 & 5.7 & -76.6 & 7.37 & SRNL-STI-2009-697 & DWPF Simulated Melter Feed \\
\hline Polyacrylic Acid, $1800 \mathrm{MW}$ & SB6 SME-1 & 1,000 & 28.2 & 20.2 & 4.25 & 4.65 & 9.4 & np & SRNL-STI-2011-674 & DWPF Simulated Melter Feed \\
\hline Polyacrylic Acid, $1800 \mathrm{MW}$ & SB6 SME-2 & 1,000 & 30.9 & 22.3 & 5.98 & 6.08 & 1.7 & $\mathrm{np}$ & SRNL-STI-2011-674 & \begin{tabular}{|l} 
DWPF Simulated Melter Feed \\
\end{tabular} \\
\hline Polyacrylic Acid, $1800 \mathrm{MW}$ & SB6 SME-9 & 1,000 & 48.2 & 36.4 & 31.2 & 30.9 & -1.0 & np & SRNL-STI-2011-674 & DWPF Simulated Melter Feed \\
\hline Polyacrylic Acid, $1800 \mathrm{MW}$ & SB6 SME-4 & 1,000 & 36 & 26.4 & 10.8 & 10.5 & -2.8 & np & SRNL-STI-2011-674 & DWPF Simulated Melter Feed \\
\hline Polyacrylic Acid, $1800 \mathrm{MW}$ & SB6 SME-8 & 1,000 & 46.4 & 34.9 & 26.4 & 25.6 & -3.0 & np & SRNL-STI-2011-674 & DWPF Simulated Melter Feed \\
\hline Polyacrylic Acid, $1800 \mathrm{MW}$ & SB6 SME-3 & 1,000 & 33 & 23.8 & 7.82 & 7.38 & -5.6 & np & SRNL-STI-2011-674 & DWPF Simulated Melter Feed \\
\hline Polyacrylic Acid, $1800 \mathrm{MW}$ & SB6 SME-7 & 1,000 & 43.6 & 32.4 & 24.9 & 23.4 & -6.0 & np & SRNL-STI-2011-674 & DWPF Simulated Melter Feed \\
\hline Polyacrylic Acid, 1800 MW & SB6 SME-5 & 1,000 & 38.6 & 28.3 & 14.5 & 13.5 & -6.9 & np & SRNL-STI-2011-674 & DWPF Simulated Melter Feed \\
\hline Polyacrylic Acid, 1800 MW & SB6 SME-6 & 1,000 & 41.5 & 30.8 & 19 & 17.4 & -8.4 & np & \begin{tabular}{|l|} 
SRNL-STI-2011-674 \\
\end{tabular} & \begin{tabular}{|l} 
DWPF Simulated Melter Feed \\
\end{tabular} \\
\hline Polyacrylic Acid, $1800 \mathrm{MW}$ & SB6 SME-1 & 4,000 & 28.2 & 20.2 & 4.25 & 3.75 & -11.8 & np & SRNL-STI-2011-674 & DWPF Simulated Melter Feed \\
\hline Polyacrylic Acid, $1800 \mathrm{MW}$ & SB6 SME-9 & 4,000 & 48.2 & 36.4 & 31.2 & 26.4 & -15.4 & np & SRNL-STI-2011-674 & DWPF Simulated Melter Feed \\
\hline Polyacrylic Acid, $1800 \mathrm{MW}$ & SB6 SME-2 & 4,000 & 30.9 & 22.3 & 5.98 & 5.03 & -15.9 & np & SRNL-STI-2011-674 & DWPF Simulated Melter Feed \\
\hline Polyacrylic Acid, $1800 \mathrm{MW}$ & SB6 SME-5 & 4,000 & 38.6 & 28.3 & 14.5 & 12.1 & -16.6 & np & SRNL-STI-2011-674 & DWPF Simulated Melter Feed \\
\hline Polyacrylic Acid, $1800 \mathrm{MW}$ & SB6 SME-8 & 4,000 & 46.4 & 34.9 & 26.4 & 22 & -16.7 & np & SRNL-STI-2011-674 & DWPF Simulated Melter Feed \\
\hline Polyacrylic Acid, $1800 \mathrm{MW}$ & SB6 SME-4 & 4,000 & 36 & 26.4 & 10.8 & 8.9 & -17.6 & np & SRNL-STI-2011-674 & DWPF Simulated Melter Feed \\
\hline Polyacrylic Acid, $1800 \mathrm{MW}$ & SB6 SME-9 & 7,000 & 48.2 & 36.4 & 31.2 & 25.6 & -17.9 & np & SRNL-STI-2011-674 & DWPF Simulated Melter Feed \\
\hline Polyacrylic Acid, $1800 \mathrm{MW}$ & SB4-SME-2 & 1,000 & 43.9 & 36 & 4.02 & 3.25 & -19.2 & np & SRNL-STI-2011-674 & DWPF Simulated Melter Feed \\
\hline Polyacrylic Acid, $1800 \mathrm{MW}$ & SB6 SME-3 & 4,000 & 33 & 23.8 & 7.82 & 6.27 & -19.8 & $\mathrm{np}$ & SRNL-STI-2011-674 & DWPF Simulated Melter Feed \\
\hline
\end{tabular}




\begin{tabular}{|c|c|c|c|c|c|c|c|c|c|c|}
\hline Modifier & Sample & $\begin{array}{l}\text { Mod conc, } \\
\text { ppm }\end{array}$ & $\begin{array}{c}\text { Total Solids, } \\
\%\end{array}$ & UDS, \% & \begin{tabular}{|c|} 
Yield \\
Stress \\
no Mod, \\
Pa \\
\end{tabular} & $\begin{array}{c}\text { Yield } \\
\text { Stress w } \\
\text { Mod, Pa }\end{array}$ & $\begin{array}{l}\text { Calc \% } \\
\text { Change }\end{array}$ & pH & Ref & Category \\
\hline Polyacrylic Acid, $1800 \mathrm{MW}$ & SB6 SME-6 & 4,000 & 41.5 & 30.8 & 19 & 15.1 & -20.5 & $\mathrm{np}$ & SRNL-STI-2011-674 & DWPF Simulated Melter Feed \\
\hline Polyacrylic Acid, 1800 MW & SB4-SME-5 & 1,000 & 51.9 & 42.5 & 23.8 & 18.6 & -21.8 & np & SRNL-STI-2011-674 & DWPF Simulated Melter Feed \\
\hline Polyacrylic Acid, $1800 \mathrm{MW}$ & \begin{tabular}{|l|} 
SB6 SME-7 \\
\end{tabular} & 4,000 & 43.6 & 32.4 & 24.9 & 19.3 & -22.5 & np & SRNL-STI-2011-674 & \begin{tabular}{|l} 
DWPF Simulated Melter Feed \\
\end{tabular} \\
\hline Polyacrylic Acid, $1800 \mathrm{MW}$ & SB4-SME-4 & 1,000 & 49.2 & 40.1 & 13.5 & 9.9 & -26.7 & np & SRNL-STI-2011-674 & DWPF Simulated Melter Feed \\
\hline Polyacrylic Acid, $1800 \mathrm{MW}$ & SB6 SME-5 & 7,000 & 38.6 & 28.3 & 14.5 & 10.5 & -27.6 & np & SRNL-STI-2011-674 & DWPF Simulated Melter Feed \\
\hline Polyacrylic Acid, $1800 \mathrm{MW}$ & SB4-SME-1 & 1,000 & 41.2 & 33.7 & 3.05 & 2.19 & -28.2 & $\mathrm{np}$ & SRNL-STI-2011-674 & DWPF Simulated Melter Feed \\
\hline Polyacrylic Acid, $1800 \mathrm{MW}$ & SB6 SME-3 & 7,000 & 33 & 23.8 & 7.82 & 5.46 & -30.2 & np & SRNL-STI-2011-674 & DWPF Simulated Melter Feed \\
\hline Polyacrylic Acid, 1800 MW & SB6 SME-2 & 7,000 & 30.9 & 22.3 & 5.98 & 4.17 & -30.3 & $\mathrm{np}$ & SRNL-STI-2011-674 & DWPF Simulated Melter Feed \\
\hline Polyacrylic Acid, $1800 \mathrm{MW}$ & SB6 SME-6 & 7,000 & 41.5 & 30.8 & 19 & 13.2 & -30.5 & np & SRNL-STI-2011-674 & DWPF Simulated Melter Feed \\
\hline Polyacrylic Acid, $1800 \mathrm{MW}$ & SB4-SME-3 & 1,000 & 46.7 & 38.4 & 7.49 & 5.14 & -31.4 & np & SRNL-STI-2011-674 & DWPF Simulated Melter Feed \\
\hline Polyacrylic Acid, $1800 \mathrm{MW}$ & SB6 SME-4 & 7,000 & 36 & 26.4 & 10.8 & 7.3 & -32.4 & $\mathrm{np}$ & SRNL-STI-2011-674 & DWPF Simulated Melter Feed \\
\hline Polyacrylic Acid, $1800 \mathrm{MW}$ & SB6 SME-8 & 7,000 & 46.4 & 34.9 & 26.4 & 17.7 & -33.0 & $\mathrm{np}$ & SRNL-STI-2011-674 & DWPF Simulated Melter Feed \\
\hline Polyacrylic Acid, $1800 \mathrm{MW}$ & \begin{tabular}{|l|} 
SB6 SME-7 \\
\end{tabular} & 7,000 & 43.6 & 32.4 & 24.9 & 16.3 & -34.5 & $\mathrm{np}$ & SRNL-STI-2011-674 & DWPF Simulated Melter Feed \\
\hline Polyacrylic Acid, $1800 \mathrm{MW}$ & \begin{tabular}{|l|} 
SB6 SME-1 \\
\end{tabular} & 7,000 & 28.2 & 20.2 & 4.25 & 2.78 & -34.6 & $\mathrm{np}$ & SRNL-STI-2011-674 & \begin{tabular}{|l} 
DWPF Simulated Melter Feed \\
\end{tabular} \\
\hline $\begin{array}{l}\text { Polyacrylic Acid, } 1800 \mathrm{MW} \\
\end{array}$ & \begin{tabular}{|l|} 
SB6 SME-9 \\
\end{tabular} & 10,000 & 48.2 & 36.4 & 31.2 & 19.7 & -36.9 & $\mathrm{np}$ & SRNL-STI-2011-674 & DWPF Simulated Melter Feed \\
\hline Polyacrylic Acid, 1800 MW & SB6 SME-5 & 10,000 & 38.6 & 28.3 & 14.5 & 9 & -37.9 & $\mathrm{np}$ & SRNL-STI-2011-674 & DWPF Simulated Melter Feed \\
\hline $\begin{array}{l}\text { Polyacrylic Acid, } 1800 \mathrm{MW} \\
\end{array}$ & SB6 SME-8 & 10,000 & 46.4 & 34.9 & 26.4 & 15.1 & -42.8 & $\mathrm{np}$ & SRNL-STI-2011-674 & DWPF Simulated Melter Feed \\
\hline Polyacrylic Acid, $1800 \mathrm{MW}$ & SB6 SME-7 & 10,000 & 43.6 & 32.4 & 24.9 & 14.1 & -43.4 & $\mathrm{np}$ & SRNL-STI-2011-674 & DWPF Simulated Melter Feed \\
\hline Polyacrylic Acid, $1800 \mathrm{MW}$ & SB6 SME-4 & 10,000 & 36 & 26.4 & 10.8 & 6.1 & -43.5 & $\mathrm{np}$ & SRNL-STI-2011-674 & DWPF Simulated Melter Feed \\
\hline Polyacrylic Acid, $1800 \mathrm{MW}$ & SB6 SME-6 & 10,000 & 41.5 & 30.8 & 19 & 10.7 & -43.7 & $\mathrm{np}$ & SRNL-STI-2011-674 & DWPF Simulated Melter Feed \\
\hline Polyacrylic Acid, $1800 \mathrm{MW}$ & SB6 SME-3 & 10,000 & 33 & 23.8 & 7.82 & 4.31 & -44.9 & $\mathrm{np}$ & SRNL-STI-2011-674 & DWPF Simulated Melter Feed \\
\hline Polyacrylic Acid, $1800 \mathrm{MW}$ & SB6 SME-1 & 10,000 & 28.2 & 20.2 & 4.25 & 2.15 & -49.4 & $\mathrm{np}$ & SRNL-STI-2011-674 & DWPF Simulated Melter Feed \\
\hline Polyacrylic Acid, $1800 \mathrm{MW}$ & SB6 SME-2 & 10,000 & 30.9 & 22.3 & 5.98 & 3.02 & -49.5 & $\mathrm{np}$ & SRNL-STI-2011-674 & DWPF Simulated Melter Feed \\
\hline Polyacrylic Acid, $1800 \mathrm{MW}$ & SB4-SME-2 & 4,000 & 43.9 & 36 & 4.02 & 1.35 & -66.4 & $\mathrm{np}$ & SRNL-STI-2011-674 & DWPF Simulated Melter Feed \\
\hline Polyacrylic Acid, $1800 \mathrm{MW}$ & SB4-SME-1 & 4,000 & 41.2 & 33.7 & 3.05 & 0.96 & -68.5 & np & SRNL-STI-2011-674 & DWPF Simulated Melter Feed \\
\hline $\begin{array}{l}\text { Polyacrylic Acid, } 1800 \mathrm{MW} \\
\end{array}$ & SB4-SME-4 & 4,000 & 49.2 & 40.1 & 13.5 & 3.7 & -72.6 & $\mathrm{np}$ & SRNL-STI-2011-674 & DWPF Simulated Melter Feed \\
\hline Polyacrylic Acid, 1800 MW & SB4-SME-3 & 4,000 & 46.7 & 38.4 & 7.49 & 2.04 & $\begin{array}{l}-72.8 \\
\end{array}$ & np & SRNL-STI-2011-674 & DWPF Simulated Melter Feed \\
\hline $\begin{array}{l}\text { Polyacrylic Acid, } 1800 \mathrm{MW} \\
\end{array}$ & SB4-SME-5 & 4,000 & 51.9 & 42.5 & 23.8 & 6.2 & $\begin{array}{l}-73.9 \\
\end{array}$ & $\mathrm{np}$ & SRNL-STI-2011-674 & DWPF Simulated Melter Feed \\
\hline Polyacrylic Acid, $1800 \mathrm{MW}$ & SB4-SME-1 & 7,000 & 41.2 & 33.7 & 3.05 & 0.53 & -82.6 & np & SRNL-STI-2011-674 & DWPF Simulated Melter Feed \\
\hline Polyacrylic Acid, $1800 \mathrm{MW}$ & SB4-SME-2 & 7,000 & 43.9 & 36 & 4.02 & 0.61 & -84.8 & np & SRNL-STI-2011-674 & DWPF Simulated Melter Feed \\
\hline Polyacrylic Acid, $1800 \mathrm{MW}$ & SB4-SME-3 & 7,000 & 46.7 & 38.4 & 7.49 & 0.94 & -87.4 & np & SRNL-STI-2011-674 & DWPF Simulated Melter Feed \\
\hline Polyacrylic Acid, $1800 \mathrm{MW}$ & SB4-SME-5 & 7,000 & 51.9 & 42.5 & 23.8 & 2.9 & -87.8 & $\mathrm{np}$ & SRNL-STI-2011-674 & DWPF Simulated Melter Feed \\
\hline Polyacrylic Acid, $1800 \mathrm{MW}$ & SB4-SME-4 & 7,000 & 49.2 & 40.1 & 13.5 & 1.6 & -88.1 & $\mathrm{np}$ & SRNL-STI-2011-674 & DWPF Simulated Melter Feed \\
\hline Polyacrylic Acid, $1800 \mathrm{MW}$ & SB4-SME-1 & 10,000 & 41.2 & 33.7 & 3.05 & 0.3 & -90.2 & $\mathrm{np}$ & SRNL-STI-2011-674 & DWPF Simulated Melter Feed \\
\hline Polyacrylic Acid, $1800 \mathrm{MW}$ & \begin{tabular}{|l|} 
SB4-SME-2 \\
\end{tabular} & 10,000 & 43.9 & 36 & 4.02 & 0.35 & -91.3 & $\mathrm{np}$ & SRNL-STI-2011-674 & DWPF Simulated Melter Feed \\
\hline Polyacrylic Acid, $1800 \mathrm{MW}$ & \begin{tabular}{|l|} 
SB4-SME-5 \\
\end{tabular} & 10,000 & 51.9 & 42.5 & 23.8 & 1.9 & -92.0 & $\mathrm{np}$ & SRNL-STI-2011-674 & \begin{tabular}{|l} 
DWPF Simulated Melter Feed \\
\end{tabular} \\
\hline Polyacrylic Acid, $1800 \mathrm{MW}$ & SB4-SME-3 & 10,000 & 46.7 & 38.4 & 7.49 & 0.58 & -92.3 & $\mathrm{np}$ & SRNL-STI-2011-674 & DWPF Simulated Melter Feed \\
\hline Polyacrylic Acid, $1800 \mathrm{MW}$ & SB4-SME-4 & 10,000 & 49.2 & 40.1 & 13.5 & 1 & -92.6 & $\mathrm{np}$ & SRNL-STI-2011-674 & \begin{tabular}{|l|} 
DWPF Simulated Melter Feed \\
\end{tabular} \\
\hline $\begin{array}{l}\text { Polyacrylic Acid, } 2000 \mathrm{MW} \\
\end{array}$ & SB6 SME-1 & 1,000 & 28.2 & 20.2 & 4.25 & 4.68 & 10.1 & $\mathrm{np}$ & SRNL-STI-2011-674 & DWPF Simulated Melter Feed \\
\hline
\end{tabular}




\begin{tabular}{|c|c|c|c|c|c|c|c|c|c|c|}
\hline Modifier & Sample & $\begin{array}{l}\text { Mod conc, } \\
\text { ppm }\end{array}$ & $\begin{array}{c}\text { Total Solids, } \\
\%\end{array}$ & UDS, \% & \begin{tabular}{|c|} 
Yield \\
Stress \\
no Mod, \\
Pa \\
\end{tabular} & $\begin{array}{c}\text { Yield } \\
\text { Stress w } \\
\text { Mod, Pa }\end{array}$ & $\begin{array}{l}\text { Calc \% } \\
\text { Change }\end{array}$ & pH & Ref & Category \\
\hline $\begin{array}{l}\text { Polyacrylic Acid, } 2000 \mathrm{MW} \\
\end{array}$ & SB6 SME-1 & 5,500 & 28.2 & 20.2 & 4.25 & 4.6 & 8.2 & $\mathrm{np}$ & SRNL-STI-2011-674 & DWPF Simulated Melter Feed \\
\hline Polyacrylic Acid, 2000 MW & SB6 SME-9 & 1,000 & 48.2 & 36.4 & 31.2 & 33 & 5.8 & $\mathrm{np}$ & SRNL-STI-2011-674 & DWPF Simulated Melter Feed \\
\hline Polyacrylic Acid, 2000 MW & \begin{tabular}{|l|} 
SB6 SME-3 \\
\end{tabular} & 1,000 & 33 & 23.8 & 7.82 & 8.19 & 4.7 & np & SRNL-STI-2011-674 & \begin{tabular}{|l} 
DWPF Simulated Melter Feed \\
\end{tabular} \\
\hline Polyacrylic Acid, $2000 \mathrm{MW}$ & SB6 SME-7 & 1,000 & 43.6 & 32.4 & 24.9 & 26 & 4.4 & np & SRNL-STI-2011-674 & DWPF Simulated Melter Feed \\
\hline Polyacrylic Acid, $2000 \mathrm{MW}$ & SB6 SME-2 & 1,000 & 30.9 & 22.3 & 5.98 & 6.03 & 0.8 & np & SRNL-STI-2011-674 & DWPF Simulated Melter Feed \\
\hline Polyacrylic Acid, $2000 \mathrm{MW}$ & SB6 SME-8 & 1,000 & 46.4 & 34.9 & 26.4 & 26.6 & 0.8 & np & SRNL-STI-2011-674 & DWPF Simulated Melter Feed \\
\hline Polyacrylic Acid, 2000 MW & SB6 SME-4 & 1,000 & 36 & 26.4 & 10.8 & 10.8 & 0.0 & np & SRNL-STI-2011-674 & DWPF Simulated Melter Feed \\
\hline Polyacrylic Acid, $2000 \mathrm{MW}$ & SB6 SME-6 & 1,000 & 41.5 & 30.8 & 19 & 18.8 & -1.1 & np & SRNL-STI-2011-674 & DWPF Simulated Melter Feed \\
\hline Polyacrylic Acid, $2000 \mathrm{MW}$ & SB6 SME-5 & 1,000 & 38.6 & 28.3 & 14.5 & 14.3 & -1.4 & np & SRNL-STI-2011-674 & DWPF Simulated Melter Feed \\
\hline Polyacrylic Acid, $2000 \mathrm{MW}$ & SB6 SME-3 & 5,500 & 33 & 23.8 & 7.82 & 7.7 & -1.5 & np & SRNL-STI-2011-674 & DWPF Simulated Melter Feed \\
\hline Polyacrylic Acid, $2000 \mathrm{MW}$ & SB6 SME-9 & 5,500 & 48.2 & 36.4 & 31.2 & 30.6 & -1.9 & $\mathrm{np}$ & SRNL-STI-2011-674 & DWPF Simulated Melter Feed \\
\hline Polyacrylic Acid, $2000 \mathrm{MW}$ & SB6 SME-1 & 9,500 & 28.2 & 20.2 & 4.25 & 4.1 & -3.5 & np & SRNL-STI-2011-674 & DWPF Simulated Melter Feed \\
\hline Polyacrylic Acid, $2000 \mathrm{MW}$ & \begin{tabular}{|l|} 
SB6 SME-7 \\
\end{tabular} & 5,500 & 43.6 & 32.4 & 24.9 & 23.9 & -4.0 & $\mathrm{np}$ & SRNL-STI-2011-674 & DWPF Simulated Melter Feed \\
\hline Polyacrylic Acid, 2000 MW & \begin{tabular}{|l|} 
SB6 SME-9 \\
\end{tabular} & 9,500 & 48.2 & 36.4 & 31.2 & 29.9 & -4.2 & $\mathrm{np}$ & SRNL-STI-2011-674 & \begin{tabular}{|l} 
DWPF Simulated Melter Feed \\
\end{tabular} \\
\hline Polyacrylic Acid, $2000 \mathrm{MW}$ & SB6 SME-8 & 5,500 & 46.4 & 34.9 & 26.4 & 25.1 & -4.9 & $\mathrm{np}$ & SRNL-STI-2011-674 & DWPF Simulated Melter Feed \\
\hline Polyacrylic Acid, 2000 MW & SB6 SME-2 & 5,500 & 30.9 & 22.3 & 5.98 & 5.65 & -5.5 & $\mathrm{np}$ & SRNL-STI-2011-674 & DWPF Simulated Melter Feed \\
\hline Polyacrylic Acid, 2000 MW & SB6 SME-4 & 5,500 & 36 & 26.4 & 10.8 & 10.2 & -5.6 & $\mathrm{np}$ & SRNL-STI-2011-674 & DWPF Simulated Melter Feed \\
\hline Polyacrylic Acid, 2000 MW & SB6 SME-5 & 5,500 & 38.6 & 28.3 & 14.5 & 13.3 & -8.3 & $\mathrm{np}$ & SRNL-STI-2011-674 & DWPF Simulated Melter Feed \\
\hline Polyacrylic Acid, 2000 MW & SB6 SME-7 & 9,500 & 43.6 & 32.4 & 24.9 & 22.7 & -8.8 & $\mathrm{np}$ & SRNL-STI-2011-674 & DWPF Simulated Melter Feed \\
\hline Polyacrylic Acid, $2000 \mathrm{MW}$ & SB6 SME-2 & 9,500 & 30.9 & 22.3 & 5.98 & 5.43 & -9.2 & $\mathrm{np}$ & SRNL-STI-2011-674 & DWPF Simulated Melter Feed \\
\hline Polyacrylic Acid, 2000 MW & SB4-SME-4 & 1,000 & 49.2 & 40.1 & 13.5 & 12.2 & -9.6 & $\mathrm{np}$ & SRNL-STI-2011-674 & DWPF Simulated Melter Feed \\
\hline Polyacrylic Acid, $2000 \mathrm{MW}$ & SB4-SME-1 & 1,000 & 41.2 & 33.7 & 3.05 & 2.75 & -9.8 & $\mathrm{np}$ & SRNL-STI-2011-674 & DWPF Simulated Melter Feed \\
\hline Polyacrylic Acid, $2000 \mathrm{MW}$ & SB6 SME-6 & 5,500 & 41.5 & 30.8 & 19 & 17.1 & -10.0 & $\mathrm{np}$ & SRNL-STI-2011-674 & DWPF Simulated Melter Feed \\
\hline Polyacrylic Acid, $2000 \mathrm{MW}$ & SB4-SME-2 & 1,000 & 43.9 & 36 & 4.02 & 3.56 & -11.4 & $\mathrm{np}$ & SRNL-STI-2011-674 & DWPF Simulated Melter Feed \\
\hline Polyacrylic Acid, 2000 MW & SB6 SME-8 & 9,500 & 46.4 & 34.9 & 26.4 & 23 & $\begin{array}{c}-12.9 \\
\end{array}$ & $\mathrm{np}$ & SRNL-STI-2011-674 & DWPF Simulated Melter Feed \\
\hline $\begin{array}{l}\text { Polyacrylic Acid, } 2000 \mathrm{MW} \\
\end{array}$ & SB6 SME-3 & 9,500 & 33 & 23.8 & 7.82 & 6.63 & -15.2 & $\mathrm{np}$ & SRNL-STI-2011-674 & DWPF Simulated Melter Feed \\
\hline Polyacrylic Acid, 2000 MW & SB6 SME-6 & 9,500 & 41.5 & 30.8 & 19 & 15.8 & -16.8 & np & SRNL-STI-2011-674 & DWPF Simulated Melter Feed \\
\hline $\begin{array}{l}\text { Polyacrylic Acid, } 2000 \mathrm{MW} \\
\end{array}$ & SB6 SME-4 & 9,500 & 36 & 26.4 & 10.8 & 8.9 & $\begin{array}{c}-17.6 \\
\end{array}$ & $\mathrm{np}$ & SRNL-STI-2011-674 & DWPF Simulated Melter Feed \\
\hline Polyacrylic Acid, 2000 MW & SB6 SME-2 & 14,000 & 30.9 & 22.3 & 5.98 & 4.92 & -17.7 & np & SRNL-STI-2011-674 & DWPF Simulated Melter Feed \\
\hline Polyacrylic Acid, $2000 \mathrm{MW}$ & SB4-SME-3 & 1,000 & 46.7 & 38.4 & 7.49 & 6.06 & -19.1 & np & SRNL-STI-2011-674 & DWPF Simulated Melter Feed \\
\hline Polyacrylic Acid, $2000 \mathrm{MW}$ & SB6 SME-5 & 9,500 & 38.6 & 28.3 & 14.5 & 11.6 & -20.0 & np & SRNL-STI-2011-674 & DWPF Simulated Melter Feed \\
\hline Polyacrylic Acid, $2000 \mathrm{MW}$ & SB6 SME-9 & 14,000 & 48.2 & 36.4 & 31.2 & 24.7 & -20.8 & $\mathrm{np}$ & SRNL-STI-2011-674 & DWPF Simulated Melter Feed \\
\hline Polyacrylic Acid, $2000 \mathrm{MW}$ & SB6 SME-7 & 14,000 & 43.6 & 32.4 & 24.9 & 19.5 & -21.7 & $\mathrm{np}$ & SRNL-STI-2011-674 & DWPF Simulated Melter Feed \\
\hline Polyacrylic Acid, $2000 \mathrm{MW}$ & SB6 SME-6 & 14,000 & 41.5 & 30.8 & 19 & 14.7 & -22.6 & $\mathrm{np}$ & SRNL-STI-2011-674 & DWPF Simulated Melter Feed \\
\hline Polyacrylic Acid, $2000 \mathrm{MW}$ & \begin{tabular}{|l|} 
SB6 SME-8 \\
\end{tabular} & 14,000 & 46.4 & 34.9 & 26.4 & 20.4 & -22.7 & $\mathrm{np}$ & SRNL-STI-2011-674 & DWPF Simulated Melter Feed \\
\hline Polyacrylic Acid, 2000 MW & SB6 SME-4 & 14,000 & 36 & 26.4 & 10.8 & 8.3 & -23.1 & $\mathrm{np}$ & SRNL-STI-2011-674 & DWPF Simulated Melter Feed \\
\hline Polyacrylic Acid, $2000 \mathrm{MW}$ & SB6 SME-1 & 14,000 & 28.2 & 20.2 & 4.25 & 3.17 & -25.4 & $\mathrm{np}$ & SRNL-STI-2011-674 & DWPF Simulated Melter Feed \\
\hline Polyacrylic Acid, $2000 \mathrm{MW}$ & SB6 SME-3 & 14,000 & 33 & 23.8 & 7.82 & 5.72 & -26.9 & $\mathrm{np}$ & SRNL-STI-2011-674 & \begin{tabular}{|l|} 
DWPF Simulated Melter Feed \\
\end{tabular} \\
\hline $\begin{array}{l}\text { Polyacrylic Acid, } 2000 \mathrm{MW} \\
\end{array}$ & SB6 SME-5 & 14,000 & 38.6 & 28.3 & 14.5 & 9.9 & -31.7 & $\mathrm{np}$ & SRNL-STI-2011-674 & DWPF Simulated Melter Feed \\
\hline
\end{tabular}




\begin{tabular}{|c|c|c|c|c|c|c|c|c|c|c|}
\hline Modifier & Sample & $\begin{array}{l}\text { Mod conc, } \\
\text { ppm }\end{array}$ & $\begin{array}{c}\text { Total Solids, } \\
\%\end{array}$ & UDS, \% & \begin{tabular}{|c|} 
Yield \\
Stress \\
no Mod, \\
Pa \\
\end{tabular} & $\begin{array}{c}\text { Yield } \\
\text { Stress w } \\
\text { Mod, Pa }\end{array}$ & $\begin{array}{l}\text { Calc \% } \\
\text { Change }\end{array}$ & pH & Ref & Category \\
\hline Polyacrylic Acid, $2000 \mathrm{MW}$ & \begin{tabular}{|l|} 
SB4-SME-1 \\
\end{tabular} & 5,500 & 41.2 & 33.7 & 3.05 & 1.54 & -49.5 & $\mathrm{np}$ & SRNL-STI-2011-674 & DWPF Simulated Melter Feed \\
\hline Polyacrylic Acid, $2000 \mathrm{MW}$ & \begin{tabular}{|l|} 
SB4-SME-2 \\
\end{tabular} & 5,500 & 43.9 & 36 & 4.02 & 1.9 & -52.7 & np & SRNL-STI-2011-674 & \begin{tabular}{|l|} 
DWPF Simulated Melter Feed \\
\end{tabular} \\
\hline Polyacrylic Acid, 2000 MW & \begin{tabular}{|l|} 
SB4-SME-4 \\
\end{tabular} & 5,500 & 49.2 & 40.1 & 13.5 & 6.2 & -54.1 & np & SRNL-STI-2011-674 & \begin{tabular}{|l} 
DWPF Simulated Melter Feed \\
\end{tabular} \\
\hline Polyacrylic Acid, $2000 \mathrm{MW}$ & SB4-SME-3 & 5,500 & 46.7 & 38.4 & 7.49 & 3.1 & -58.6 & np & SRNL-STI-2011-674 & DWPF Simulated Melter Feed \\
\hline Polyacrylic Acid, $2000 \mathrm{MW}$ & SB4-SME-1 & 9,500 & 41.2 & 33.7 & 3.05 & 0.84 & -72.5 & np & SRNL-STI-2011-674 & DWPF Simulated Melter Feed \\
\hline Polyacrylic Acid, $2000 \mathrm{MW}$ & SB4-SME-1 & 14,000 & 41.2 & 33.7 & 3.05 & 0.72 & -76.4 & $\mathrm{np}$ & SRNL-STI-2011-674 & DWPF Simulated Melter Feed \\
\hline Polyacrylic Acid, 2000 MW & SB4-SME-2 & 9,500 & 43.9 & 36 & 4.02 & 0.93 & -76.9 & np & SRNL-STI-2011-674 & DWPF Simulated Melter Feed \\
\hline Polyacrylic Acid, 2000 MW & SB4-SME-3 & 9,500 & 46.7 & 38.4 & 7.49 & 1.63 & -78.2 & $\mathrm{np}$ & SRNL-STI-2011-674 & DWPF Simulated Melter Feed \\
\hline Polyacrylic Acid, $2000 \mathrm{MW}$ & SB4-SME-3 & 14,000 & 46.7 & 38.4 & 7.49 & 1.37 & -81.7 & np & SRNL-STI-2011-674 & DWPF Simulated Melter Feed \\
\hline Polyacrylic Acid, $2000 \mathrm{MW}$ & SB4-SME-2 & 14,000 & 43.9 & 36 & 4.02 & 0.62 & -84.6 & np & SRNL-STI-2011-674 & DWPF Simulated Melter Feed \\
\hline Polyacrylic Acid, $2000 \mathrm{MW}$ & SB4-SME-4 & 9,500 & 49.2 & 40.1 & 13.5 & 1.9 & -85.9 & $\mathrm{np}$ & SRNL-STI-2011-674 & DWPF Simulated Melter Feed \\
\hline Polyacrylic Acid, $2000 \mathrm{MW}$ & SB4-SME-4 & 14,000 & 49.2 & 40.1 & 13.5 & 1.7 & -87.4 & $\mathrm{np}$ & SRNL-STI-2011-674 & DWPF Simulated Melter Feed \\
\hline Polyacrylic Acid, $5000 \mathrm{MW}$ & \begin{tabular}{|l|} 
SB6 SME-1 \\
\end{tabular} & 1,000 & 28.2 & 20.2 & 4.25 & 4.82 & 13.4 & $\mathrm{np}$ & SRNL-STI-2011-674 & DWPF Simulated Melter Feed \\
\hline Polyacrylic Acid, $5000 \mathrm{MW}$ & \begin{tabular}{|l|} 
SB6 SME-9 \\
\end{tabular} & 1,000 & 48.2 & 36.4 & 31.2 & 32.9 & 5.4 & $\mathrm{np}$ & SRNL-STI-2011-674 & \begin{tabular}{|l} 
DWPF Simulated Melter Feed \\
\end{tabular} \\
\hline Polyacrylic Acid, $5000 \mathrm{MW}$ & SB6 SME-2 & 1,000 & 30.9 & 22.3 & 5.98 & 6.2 & 3.7 & $\mathrm{np}$ & SRNL-STI-2011-674 & DWPF Simulated Melter Feed \\
\hline Polyacrylic Acid, 5000 MW & SB6 SME-8 & 1,000 & 46.4 & 34.9 & 26.4 & 27.3 & 3.4 & $\mathrm{np}$ & SRNL-STI-2011-674 & DWPF Simulated Melter Feed \\
\hline $\begin{array}{l}\text { Polyacrylic Acid, } 5000 \mathrm{MW} \\
\end{array}$ & SB6 SME-2 & 5,500 & 30.9 & 22.3 & 5.98 & 6.11 & 2.2 & $\mathrm{np}$ & SRNL-STI-2011-674 & DWPF Simulated Melter Feed \\
\hline Polyacrylic Acid, $5000 \mathrm{MW}$ & SB6 SME-7 & 1,000 & 43.6 & 32.4 & 24.9 & 25.4 & 2.0 & $\mathrm{np}$ & SRNL-STI-2011-674 & \begin{tabular}{|l|} 
DWPF Simulated Melter Feed \\
\end{tabular} \\
\hline Polyacrylic Acid, $5000 \mathrm{MW}$ & SB6 SME-6 & 1,000 & 41.5 & 30.8 & 19 & 19.1 & 0.5 & $\mathrm{np}$ & SRNL-STI-2011-674 & DWPF Simulated Melter Feed \\
\hline Polyacrylic Acid, $5000 \mathrm{MW}$ & SB6 SME-3 & 1,000 & 33 & 23.8 & 7.82 & 7.86 & 0.5 & $\mathrm{np}$ & SRNL-STI-2011-674 & DWPF Simulated Melter Feed \\
\hline Polyacrylic Acid, $5000 \mathrm{MW}$ & SB6 SME-4 & 1,000 & 36 & 26.4 & 10.8 & 10.8 & 0.0 & $\mathrm{np}$ & SRNL-STI-2011-674 & DWPF Simulated Melter Feed \\
\hline Polyacrylic Acid, $5000 \mathrm{MW}$ & SB6 SME-9 & 5,500 & 48.2 & 36.4 & 31.2 & 31 & -0.6 & $\mathrm{np}$ & SRNL-STI-2011-674 & DWPF Simulated Melter Feed \\
\hline Polyacrylic Acid, $5000 \mathrm{MW}$ & SB6 SME-5 & 1,000 & 38.6 & 28.3 & 14.5 & 14 & -3.4 & $\mathrm{np}$ & SRNL-STI-2011-674 & DWPF Simulated Melter Feed \\
\hline Polyacrylic Acid, $5000 \mathrm{MW}$ & SB6 SME-9 & 14,000 & 48.2 & 36.4 & 31.2 & 29.7 & -4.8 & $\mathrm{np}$ & SRNL-STI-2011-674 & DWPF Simulated Melter Feed \\
\hline Polyacrylic Acid, 5000 MW & SB6 SME-7 & 9,500 & 43.6 & 32.4 & 24.9 & 23.6 & -5.2 & np & SRNL-STI-2011-674 & DWPF Simulated Melter Feed \\
\hline $\begin{array}{l}\text { Polyacrylic Acid, } 5000 \mathrm{MW} \\
\end{array}$ & SB6 SME-1 & 5,500 & 28.2 & 20.2 & 4.25 & 4.02 & -5.4 & $\mathrm{np}$ & SRNL-STI-2011-674 & DWPF Simulated Melter Feed \\
\hline Polyacrylic Acid, 5000 MW & SB4-SME-5 & 1,000 & 51.9 & 42.5 & 23.8 & 22.4 & -5.9 & np & SRNL-STI-2011-674 & DWPF Simulated Melter Feed \\
\hline $\begin{array}{l}\text { Polyacrylic Acid, } 5000 \mathrm{MW} \\
\end{array}$ & SB6 SME-8 & 5,500 & 46.4 & 34.9 & 26.4 & 24.8 & -6.1 & $\mathrm{np}$ & SRNL-STI-2011-674 & DWPF Simulated Melter Feed \\
\hline Polyacrylic Acid, $5000 \mathrm{MW}$ & SB4-SME-1 & 1,000 & 41.2 & 33.7 & 3.05 & 2.85 & -6.6 & np & SRNL-STI-2011-674 & DWPF Simulated Melter Feed \\
\hline Polyacrylic Acid, $5000 \mathrm{MW}$ & SB4-SME-4 & 1,000 & 49.2 & 40.1 & 13.5 & 12.5 & -7.4 & np & SRNL-STI-2011-674 & DWPF Simulated Melter Feed \\
\hline Polyacrylic Acid, $5000 \mathrm{MW}$ & SB6 SME-9 & 9,500 & 48.2 & 36.4 & 31.2 & 28.7 & -8.0 & np & SRNL-STI-2011-674 & DWPF Simulated Melter Feed \\
\hline Polyacrylic Acid, 5000 MW & SB6 SME-6 & 5,500 & 41.5 & 30.8 & 19 & 17.2 & -9.5 & $\mathrm{np}$ & SRNL-STI-2011-674 & DWPF Simulated Melter Feed \\
\hline Polyacrylic Acid, $5000 \mathrm{MW}$ & SB4-SME-2 & 1,000 & 43.9 & 36 & 4.02 & 3.62 & -10.0 & $\mathrm{np}$ & SRNL-STI-2011-674 & DWPF Simulated Melter Feed \\
\hline Polyacrylic Acid, $5000 \mathrm{MW}$ & SB6 SME-3 & 5,500 & 33 & 23.8 & 7.82 & 6.98 & -10.7 & $\mathrm{np}$ & SRNL-STI-2011-674 & DWPF Simulated Melter Feed \\
\hline Polyacrylic Acid, $5000 \mathrm{MW}$ & \begin{tabular}{|l|} 
SB6 SME-4 \\
\end{tabular} & 5,500 & 36 & 26.4 & 10.8 & 9.6 & -11.1 & $\mathrm{np}$ & SRNL-STI-2011-674 & DWPF Simulated Melter Feed \\
\hline Polyacrylic Acid, $5000 \mathrm{MW}$ & \begin{tabular}{|l|} 
SB6 SME-2 \\
\end{tabular} & 9,500 & 30.9 & 22.3 & 5.98 & 5.24 & -12.4 & $\mathrm{np}$ & SRNL-STI-2011-674 & \begin{tabular}{|l} 
DWPF Simulated Melter Feed \\
\end{tabular} \\
\hline Polyacrylic Acid, $5000 \mathrm{MW}$ & SB6 SME-7 & 5,500 & 43.6 & 32.4 & 24.9 & 21.8 & -12.4 & $\mathrm{np}$ & SRNL-STI-2011-674 & DWPF Simulated Melter Feed \\
\hline Polyacrylic Acid, $5000 \mathrm{MW}$ & SB6 SME-5 & 5,500 & 38.6 & 28.3 & 14.5 & 12.2 & -15.9 & $\mathrm{np}$ & SRNL-STI-2011-674 & \begin{tabular}{|l|} 
DWPF Simulated Melter Feed \\
\end{tabular} \\
\hline $\begin{array}{l}\text { Polyacrylic Acid, } 5000 \mathrm{MW} \\
\end{array}$ & SB4-SME-3 & 1,000 & 46.7 & 38.4 & 7.49 & 6.3 & -15.9 & $\mathrm{np}$ & SRNL-STI-2011-674 & DWPF Simulated Melter Feed \\
\hline
\end{tabular}




\begin{tabular}{|c|c|c|c|c|c|c|c|c|c|c|}
\hline Modifier & Sample & $\begin{array}{l}\text { Mod conc, } \\
\text { ppm }\end{array}$ & $\begin{array}{c}\text { Total Solids, } \\
\%\end{array}$ & UDS, \% & \begin{tabular}{|c|} 
Yield \\
Stress \\
no Mod, \\
Pa \\
\end{tabular} & $\begin{array}{c}\text { Yield } \\
\text { Stress w } \\
\text { Mod, Pa }\end{array}$ & $\begin{array}{l}\text { Calc \% } \\
\text { Change }\end{array}$ & pH & Ref & Category \\
\hline $\begin{array}{l}\text { Polyacrylic Acid, } 5000 \mathrm{MW} \\
\end{array}$ & SB6 SME-4 & 9,500 & 36 & 26.4 & 10.8 & 8.9 & -17.6 & np & SRNL-STI-2011-674 & DWPF Simulated Melter Feed \\
\hline Polyacrylic Acid, $5000 \mathrm{MW}$ & SB6 SME-1 & 9,500 & 28.2 & 20.2 & 4.25 & 3.49 & -17.9 & np & SRNL-STI-2011-674 & \begin{tabular}{|l|} 
DWPF Simulated Melter Feed \\
\end{tabular} \\
\hline Polyacrylic Acid, $5000 \mathrm{MW}$ & \begin{tabular}{|l|} 
SB6 SME-8 \\
\end{tabular} & 9,500 & 46.4 & 34.9 & 26.4 & 21.3 & $\begin{array}{l}-19.3 \\
\end{array}$ & np & SRNL-STI-2011-674 & \begin{tabular}{|l} 
DWPF Simulated Melter Feed \\
\end{tabular} \\
\hline Polyacrylic Acid, $5000 \mathrm{MW}$ & SB6 SME-6 & 9,500 & 41.5 & 30.8 & 19 & 15.3 & -19.5 & np & SRNL-STI-2011-674 & DWPF Simulated Melter Feed \\
\hline Polyacrylic Acid, $5000 \mathrm{MW}$ & SB6 SME-5 & 9,500 & 38.6 & 28.3 & 14.5 & 11.5 & -20.7 & np & SRNL-STI-2011-674 & DWPF Simulated Melter Feed \\
\hline Polyacrylic Acid, $5000 \mathrm{MW}$ & SB6 SME-3 & 9,500 & 33 & 23.8 & 7.82 & 6.07 & -22.4 & np & SRNL-STI-2011-674 & DWPF Simulated Melter Feed \\
\hline Polyacrylic Acid, $5000 \mathrm{MW}$ & SB6 SME-8 & 14,000 & 46.4 & 34.9 & 26.4 & 19.7 & -25.4 & np & SRNL-STI-2011-674 & DWPF Simulated Melter Feed \\
\hline Polyacrylic Acid, $5000 \mathrm{MW}$ & SB6 SME-1 & 14,000 & 28.2 & 20.2 & 4.25 & 3.05 & -28.2 & $\mathrm{np}$ & SRNL-STI-2011-674 & DWPF Simulated Melter Feed \\
\hline Polyacrylic Acid, $5000 \mathrm{MW}$ & SB6 SME-2 & 14,000 & 30.9 & 22.3 & 5.98 & 4.29 & -28.3 & np & SRNL-STI-2011-674 & DWPF Simulated Melter Feed \\
\hline Polyacrylic Acid, $5000 \mathrm{MW}$ & SB4-SME-5 & 5,500 & 51.9 & 42.5 & 23.8 & 16.8 & -29.4 & $\mathrm{np}$ & SRNL-STI-2011-674 & DWPF Simulated Melter Feed \\
\hline Polyacrylic Acid, $5000 \mathrm{MW}$ & SB6 SME-6 & 14,000 & 41.5 & 30.8 & 19 & 13 & -31.6 & np & SRNL-STI-2011-674 & DWPF Simulated Melter Feed \\
\hline Polyacrylic Acid, $5000 \mathrm{MW}$ & SB4-SME-2 & 5,500 & 43.9 & 36 & 4.02 & 2.72 & -32.3 & $\mathrm{np}$ & SRNL-STI-2011-674 & DWPF Simulated Melter Feed \\
\hline Polyacrylic Acid, $5000 \mathrm{MW}$ & SB4-SME-3 & 5,500 & 46.7 & 38.4 & 7.49 & 4.96 & -33.8 & $\mathrm{np}$ & SRNL-STI-2011-674 & DWPF Simulated Melter Feed \\
\hline Polyacrylic Acid, $5000 \mathrm{MW}$ & SB6 SME-3 & 14,000 & 33 & 23.8 & 7.82 & 5.15 & -34.1 & np & SRNL-STI-2011-674 & \begin{tabular}{|l} 
DWPF Simulated Melter Feed \\
\end{tabular} \\
\hline Polyacrylic Acid, $5000 \mathrm{MW}$ & SB6 SME-4 & 14,000 & 36 & 26.4 & 10.8 & 7 & -35.2 & $\mathrm{np}$ & SRNL-STI-2011-674 & DWPF Simulated Melter Feed \\
\hline Polyacrylic Acid, 5000 MW & SB4-SME-4 & 5,500 & 49.2 & 40.1 & 13.5 & 8.6 & -36.3 & np & SRNL-STI-2011-674 & DWPF Simulated Melter Feed \\
\hline $\begin{array}{l}\text { Polyacrylic Acid, } 5000 \mathrm{MW} \\
\end{array}$ & SB6 SME-7 & 14,000 & 43.6 & 32.4 & 24.9 & 14.8 & -40.6 & $\mathrm{np}$ & SRNL-STI-2011-674 & \begin{tabular}{|l|} 
DWPF Simulated Melter Feed \\
\end{tabular} \\
\hline Polyacrylic Acid, $5000 \mathrm{MW}$ & SB6 SME-5 & 14,000 & 38.6 & 28.3 & 14.5 & 8.6 & -40.7 & $\mathrm{np}$ & SRNL-STI-2011-674 & \begin{tabular}{|l|} 
DWPF Simulated Melter Feed \\
\end{tabular} \\
\hline Polyacrylic Acid, $5000 \mathrm{MW}$ & SB4-SME-1 & 5,500 & 41.2 & 33.7 & 3.05 & 1.67 & -45.2 & $\mathrm{np}$ & SRNL-STI-2011-674 & \begin{tabular}{|l|} 
DWPF Simulated Melter Feed \\
\end{tabular} \\
\hline Polyacrylic Acid, $5000 \mathrm{MW}$ & SB4-SME-4 & 9,500 & 49.2 & 40.1 & 13.5 & 6 & -55.6 & $\mathrm{np}$ & SRNL-STI-2011-674 & DWPF Simulated Melter Feed \\
\hline Polyacrylic Acid, $5000 \mathrm{MW}$ & SB4-SME-1 & 9,500 & 41.2 & 33.7 & 3.05 & 1.14 & -62.6 & $\mathrm{np}$ & SRNL-STI-2011-674 & DWPF Simulated Melter Feed \\
\hline Polyacrylic Acid, $5000 \mathrm{MW}$ & SB4-SME-2 & 9,500 & 43.9 & 36 & 4.02 & 1.48 & -63.2 & $\mathrm{np}$ & SRNL-STI-2011-674 & DWPF Simulated Melter Feed \\
\hline Polyacrylic Acid, $5000 \mathrm{MW}$ & SB4-SME-5 & 9,500 & 51.9 & 42.5 & 23.8 & 8.4 & -64.7 & $\mathrm{np}$ & SRNL-STI-2011-674 & DWPF Simulated Melter Feed \\
\hline Polyacrylic Acid, $5000 \mathrm{MW}$ & SB4-SME-3 & 9,500 & 46.7 & 38.4 & 7.49 & 2.49 & -66.8 & $\mathrm{np}$ & SRNL-STI-2011-674 & DWPF Simulated Melter Feed \\
\hline Polyacrylic Acid, $5000 \mathrm{MW}$ & SB4-SME-4 & 14,000 & 49.2 & 40.1 & 13.5 & 3 & $\begin{array}{l}-77.8 \\
\end{array}$ & $\mathrm{np}$ & SRNL-STI-2011-674 & DWPF Simulated Melter Feed \\
\hline $\begin{array}{l}\text { Polyacrylic Acid, } 5000 \mathrm{MW} \\
\end{array}$ & SB4-SME-1 & 14,000 & 41.2 & 33.7 & 3.05 & 0.67 & -78.0 & $\mathrm{np}$ & SRNL-STI-2011-674 & DWPF Simulated Melter Feed \\
\hline Polyacrylic Acid, 5000 MW & SB4-SME-5 & 14,000 & 51.9 & 42.5 & 23.8 & 5 & -79.0 & np & SRNL-STI-2011-674 & DWPF Simulated Melter Feed \\
\hline $\begin{array}{l}\text { Polyacrylic Acid, } 5000 \mathrm{MW} \\
\end{array}$ & SB4-SME-3 & 14,000 & 46.7 & 38.4 & 7.49 & 1.33 & -82.2 & $\mathrm{np}$ & SRNL-STI-2011-674 & DWPF Simulated Melter Feed \\
\hline Polyacrylic Acid, $5000 \mathrm{MW}$ & SB4-SME-2 & 14,000 & 43.9 & 36 & 4.02 & 0.71 & -82.3 & np & SRNL-STI-2011-674 & DWPF Simulated Melter Feed \\
\hline Pomosperse AL36 & AZ-101 & 1,000 & np & np & 3.22 & 2.93 & -9.0 & 12.23 & SRNL-GPD-2004-40 & Hanford Simulated Waste \\
\hline Pomosperse AL36 & FIU AZ-101 & 1,000 & np & np & 10.36 & 8.92 & -13.9 & 9.85 & SRNL-GPD-2004-40 & Hanford Simulated Waste \\
\hline Recover & SB6-1,2,3,4 SME & 1,093 & 46.9 & 37.2 & 2.2 & 2.43 & 10.5 & 6.02 & SRNL-STI-2009-697 & DWPF Simulated Melter Feed \\
\hline Recover & SB6 SME-1 & 5,500 & 28.2 & 20.2 & 4.25 & 4.55 & 7.1 & $\mathrm{np}$ & SRNL-STI-2011-674 & DWPF Simulated Melter Feed \\
\hline Recover & SB6 SME-8 & 1,000 & 46.4 & 34.9 & 26.4 & 28.2 & 6.8 & $\mathrm{np}$ & SRNL-STI-2011-674 & DWPF Simulated Melter Feed \\
\hline Recover & SB6 SME-7 & 1,000 & 43.6 & 32.4 & 24.9 & 26.2 & 5.2 & $\mathrm{np}$ & SRNL-STI-2011-674 & DWPF Simulated Melter Feed \\
\hline Recover & SB6 SME-6 & 1,000 & 41.5 & 30.8 & 19 & 19.9 & 4.7 & $\mathrm{np}$ & SRNL-STI-2011-674 & DWPF Simulated Melter Feed \\
\hline Recover & SB6 SME-1 & 9,500 & 28.2 & 20.2 & 4.25 & 4.45 & 4.7 & $\mathrm{np}$ & SRNL-STI-2011-674 & DWPF Simulated Melter Feed \\
\hline Recover & SB6 SME-2 & 1,000 & 30.9 & 22.3 & 5.98 & 6.23 & 4.2 & np & SRNL-STI-2011-674 & \begin{tabular}{|l|} 
DWPF Simulated Melter Feed \\
\end{tabular} \\
\hline Recover & SB6 SME-1 & 1,000 & 28.2 & 20.2 & 4.25 & 4.42 & 4.0 & $\mathrm{np}$ & SRNL-STI-2011-674 & DWPF Simulated Melter Feed \\
\hline
\end{tabular}




\begin{tabular}{|c|c|c|c|c|c|c|c|c|c|c|}
\hline Modifier & Sample & $\begin{array}{l}\text { Mod conc, } \\
\text { ppm }\end{array}$ & $\begin{array}{c}\text { Total Solids, } \\
\%\end{array}$ & UDS, \% & \begin{tabular}{|c|} 
Yield \\
Stress \\
no Mod, \\
Pa \\
\end{tabular} & $\begin{array}{c}\text { Yield } \\
\text { Stress w } \\
\text { Mod, Pa }\end{array}$ & $\begin{array}{l}\text { Calc \% } \\
\text { Change }\end{array}$ & pH & Ref & Category \\
\hline Recover & SB6 SME-8 & 5,500 & 46.4 & 34.9 & 26.4 & 27.3 & 3.4 & np & SRNL-STI-2011-674 & DWPF Simulated Melter Feed \\
\hline Recover & SB6 SME-1 & 14,000 & 28.2 & 20.2 & 4.25 & 4.39 & 3.3 & np & SRNL-STI-2011-674 & DWPF Simulated Melter Feed \\
\hline Recover & SB6 SME-3 & 14,000 & 33 & 23.8 & 7.82 & 8.04 & 2.8 & np & SRNL-STI-2011-674 & DWPF Simulated Melter Feed \\
\hline Recover & SB6 SME-9 & 1,000 & 48.2 & 36.4 & 31.2 & 31.9 & 2.2 & np & SRNL-STI-2011-674 & DWPF Simulated Melter Feed \\
\hline Recover & SB6 SME-2 & 14,000 & 30.9 & 22.3 & 5.98 & 6.1 & 2.0 & np & SRNL-STI-2011-674 & DWPF Simulated Melter Feed \\
\hline Recover & SB6 SME-2 & 5,500 & 30.9 & 22.3 & 5.98 & 5.98 & 0.0 & np & SRNL-STI-2011-674 & DWPF Simulated Melter Feed \\
\hline Recover & SB6 SME-9 & 5,500 & 48.2 & 36.4 & 31.2 & 31 & -0.6 & $\mathrm{np}$ & SRNL-STI-2011-674 & DWPF Simulated Melter Feed \\
\hline Recover & SB6 SME-2 & 9,500 & 30.9 & 22.3 & 5.98 & 5.93 & -0.8 & np & SRNL-STI-2011-674 & DWPF Simulated Melter Feed \\
\hline Recover & SB6 SME-4 & 1,000 & 36 & 26.4 & 10.8 & 10.7 & -0.9 & np & SRNL-STI-2011-674 & DWPF Simulated Melter Feed \\
\hline Recover & SB6 SME-3 & 1,000 & 33 & 23.8 & 7.82 & 7.73 & -1.2 & np & SRNL-STI-2011-674 & DWPF Simulated Melter Feed \\
\hline Recover & SB6 SME-7 & 5,500 & 43.6 & 32.4 & 24.9 & 24.6 & -1.2 & np & SRNL-STI-2011-674 & DWPF Simulated Melter Feed \\
\hline Recover & SB6 SME-7 & 14,000 & 43.6 & 32.4 & 24.9 & 24.6 & -1.2 & np & SRNL-STI-2011-674 & DWPF Simulated Melter Feed \\
\hline Recover & SB6 SME-5 & 5,500 & 38.6 & 28.3 & 14.5 & 14.3 & -1.4 & np & SRNL-STI-2011-674 & DWPF Simulated Melter Feed \\
\hline Recover & SB6 SME-8 & 9,500 & 46.4 & 34.9 & 26.4 & 25.8 & -2.3 & np & SRNL-STI-2011-674 & DWPF Simulated Melter Feed \\
\hline Recover & SB6 SME-3 & 5,500 & 33 & 23.8 & 7.82 & 7.64 & -2.3 & np & SRNL-STI-2011-674 & DWPF Simulated Melter Feed \\
\hline Recover & SB6 SME-9 & 9,500 & 48.2 & 36.4 & 31.2 & 30.4 & -2.6 & np & SRNL-STI-2011-674 & DWPF Simulated Melter Feed \\
\hline Recover & SB6 SME-9 & 14,000 & 48.2 & 36.4 & 31.2 & 30.4 & -2.6 & $\mathrm{np}$ & \begin{tabular}{|l|} 
SRNL-STI-2011-674 \\
\end{tabular} & DWPF Simulated Melter Feed \\
\hline Recover & SB6 SME-4 & 5,500 & 36 & 26.4 & 10.8 & 10.5 & -2.8 & np & SRNL-STI-2011-674 & DWPF Simulated Melter Feed \\
\hline Recover & SB6 SME-7 & 9,500 & 43.6 & 32.4 & 24.9 & 24.2 & -2.8 & $\mathrm{np}$ & SRNL-STI-2011-674 & DWPF Simulated Melter Feed \\
\hline Recover & 09-SB5-23 SME & 1,044 & 49.6 & 35.1 & 24.4 & 23.7 & -2.9 & 7.37 & SRNL-STI-2009-697 & DWPF Simulated Melter Feed \\
\hline Recover & SB4-SME-2 & 1,000 & 43.9 & 36 & 4.02 & 3.89 & -3.2 & np & SRNL-STI-2011-674 & DWPF Simulated Melter Feed \\
\hline Recover & SB6 SME-8 & 14,000 & 46.4 & 34.9 & 26.4 & 25.5 & -3.4 & $\mathrm{np}$ & SRNL-STI-2011-674 & DWPF Simulated Melter Feed \\
\hline Recover & SB6 SME-4 & 9,500 & 36 & 26.4 & 10.8 & 10.4 & -3.7 & np & SRNL-STI-2011-674 & DWPF Simulated Melter Feed \\
\hline Recover & \begin{tabular}{|l|} 
RuRhHg 1-9 Decanted \\
SME
\end{tabular} & 1,064 & np & np & 72.5 & 69.7 & -3.9 & 4.59 & SRNL-STI-2009-697 & DWPF Simulated Melter Feed \\
\hline Recover & 08-SB5-12/13 SME & 990 & 44.7 & 34.4 & 6.6 & 6.3 & -4.5 & 6.27 & SRNL-STI-2009-697 & DWPF Simulated Melter Feed \\
\hline Recover & 09-SB5-24 SME & 1,115 & 48.8 & 34.5 & 5.22 & 4.98 & -4.6 & np & \begin{tabular}{|l|} 
SRNL-STI-2009-697 \\
\end{tabular} & DWPF Simulated Melter Feed \\
\hline Recover & SB6 SME-4 & 14,000 & 36 & 26.4 & 10.8 & 10.3 & -4.6 & np & SRNL-STI-2011-674 & DWPF Simulated Melter Feed \\
\hline Recover & SB6 SME-6 & 9,500 & 41.5 & 30.8 & 19 & 18.1 & -4.7 & np & SRNL-STI-2011-674 & DWPF Simulated Melter Feed \\
\hline Recover & SB6 SME-3 & 9,500 & 33 & 23.8 & 7.82 & 7.43 & -5.0 & np & SRNL-STI-2011-674 & DWPF Simulated Melter Feed \\
\hline Recover & SB6 SME-6 & 5,500 & 41.5 & 30.8 & 19 & 17.9 & -5.8 & np & SRNL-STI-2011-674 & DWPF Simulated Melter Feed \\
\hline Recover & SB6 SME-5 & 14,000 & 38.6 & 28.3 & 14.5 & 13.6 & -6.2 & np & SRNL-STI-2011-674 & DWPF Simulated Melter Feed \\
\hline Recover & SB6 SME-5 & 1,000 & 38.6 & 28.3 & 14.5 & 13.3 & -8.3 & np & SRNL-STI-2011-674 & DWPF Simulated Melter Feed \\
\hline Recover & SB6 SME-6 & 14,000 & 41.5 & 30.8 & 19 & 17.4 & -8.4 & np & SRNL-STI-2011-674 & DWPF Simulated Melter Feed \\
\hline Recover & SB4-SME-3 & 1,000 & 46.7 & 38.4 & 7.49 & 6.8 & -9.2 & np & SRNL-STI-2011-674 & DWPF Simulated Melter Feed \\
\hline Recover & SB6 SME-5 & 9,500 & 38.6 & 28.3 & 14.5 & 13.1 & -9.7 & $\mathrm{np}$ & SRNL-STI-2011-674 & DWPF Simulated Melter Feed \\
\hline Recover & RuRhHg 1-9 SME & 1,007 & 51.55 & 42 & 13.4 & 12 & -10.4 & 4.7 & SRNL-STI-2009-697 & DWPF Simulated Melter Feed \\
\hline Recover & SB4-SME-1 & 1,000 & 41.2 & 33.7 & 3.05 & 2.73 & -10.5 & np & SRNL-STI-2011-674 & DWPF Simulated Melter Feed \\
\hline Recover & SB4-SME-4 & 1,000 & 49.2 & 40.1 & 13.5 & 12 & -11.1 & np & SRNL-STI-2011-674 & DWPF Simulated Melter Feed \\
\hline
\end{tabular}




\begin{tabular}{|c|c|c|c|c|c|c|c|c|c|c|}
\hline Modifier & Sample & $\begin{array}{l}\text { Mod conc, } \\
\text { ppm }\end{array}$ & $\begin{array}{c}\text { Total Solids, } \\
\%\end{array}$ & UDS, \% & $\begin{array}{c}\text { Yield } \\
\text { Stress } \\
\text { no Mod, } \\
\text { Pa } \\
\end{array}$ & $\begin{array}{c}\text { Yield } \\
\text { Stress w } \\
\text { Mod, Pa }\end{array}$ & $\begin{array}{l}\text { Calc \% } \\
\text { Change }\end{array}$ & $\mathbf{p H}$ & Ref & Category \\
\hline Recover & SB6-1,2,3,4 SME & 6,103 & 46.9 & 37.2 & 2.2 & 1.91 & -13.2 & 6.02 & SRNL-STI-2009-697 & DWPF Simulated Melter Feed \\
\hline Recover & SB4-SME-2 & 5,500 & 43.9 & 36 & 4.02 & 3.43 & -14.7 & $\mathrm{np}$ & SRNL-STI-2011-674 & DWPF Simulated Melter Feed \\
\hline Recover & $\begin{array}{l}\text { RuRhHg 1-9 Decanted } \\
\text { SME }\end{array}$ & 6,041 & np & np & 72.5 & 59 & $\begin{array}{c}-18.6 \\
\end{array}$ & 4.59 & SRNL-STI-2009-697 & DWPF Simulated Melter Feed \\
\hline Recover & 08-SB5-12/13 SME & 6,174 & 44.7 & 34.4 & 6.6 & 5.3 & -19.7 & 6.27 & SRNL-STI-2009-697 & DWPF Simulated Melter Feed \\
\hline Recover & 09-SB5-23 SME & 6,104 & 49.6 & 35.1 & 24.4 & 19.5 & -20.1 & 7.37 & SRNL-STI-2009-697 & DWPF Simulated Melter Feed \\
\hline Recover & SB4-SME-1 & 5,500 & 41.2 & 33.7 & 3.05 & 2.39 & -21.6 & np & SRNL-STI-2011-674 & DWPF Simulated Melter Feed \\
\hline Recover & 09-SB5-24 SME & 6,010 & 48.8 & 34.5 & 5.22 & 4.06 & -22.2 & np & SRNL-STI-2009-697 & DWPF Simulated Melter Feed \\
\hline Recover & $\begin{array}{l}\text { RuRhHg 1-9 Decanted } \\
\text { SME }\end{array}$ & 11,026 & np & np & 72.5 & 56.3 & -22.3 & 4.59 & SRNL-STI-2009-697 & DWPF Simulated Melter Feed \\
\hline Recover & RuRhHg 1-9 SME & 6,006 & 51.55 & 42 & 13.4 & 10.4 & -22.4 & 4.7 & SRNL-STI-2009-697 & DWPF Simulated Melter Feed \\
\hline Recover & SB4-SME-2 & 9,500 & 43.9 & 36 & 4.02 & 3.1 & -22.9 & $\mathrm{np}$ & SRNL-STI-2011-674 & DWPF Simulated Melter Feed \\
\hline Recover & SB4-SME-2 & 14,000 & 43.9 & 36 & 4.02 & 3.01 & -25.1 & np & SRNL-STI-2011-674 & DWPF Simulated Melter Feed \\
\hline Recover & SB6-1,2,3,4 SME & 11,107 & 46.9 & 37.2 & 2.2 & 1.62 & -26.4 & 6.02 & SRNL-STI-2009-697 & DWPF Simulated Melter Feed \\
\hline Recover & SB4-SME-4 & 5,500 & 49.2 & 40.1 & 13.5 & 9.9 & -26.7 & np & SRNL-STI-2011-674 & DWPF Simulated Melter Feed \\
\hline Recover & 08-SB5-12/13 SME & 10,897 & 44.7 & 34.4 & 6.6 & 4.7 & -28.8 & 6.27 & SRNL-STI-2009-697 & DWPF Simulated Melter Feed \\
\hline Recover & RuRhHg 1-9 SME & 10,918 & 51.55 & 42 & 13.4 & 9.4 & -29.9 & 4.7 & SRNL-STI-2009-697 & DWPF Simulated Melter Feed \\
\hline Recover & 09-SB5-24 SME & 11,156 & 48.8 & 34.5 & 5.22 & 3.64 & -30.3 & np & SRNL-STI-2009-697 & DWPF Simulated Melter Feed \\
\hline Recover & $\begin{array}{l}\text { RuRhHg 1-9 Decanted } \\
\text { SME }\end{array}$ & 16,006 & np & $\mathrm{np}$ & 72.5 & 50.4 & -30.5 & 4.59 & SRNL-STI-2009-697 & DWPF Simulated Melter Feed \\
\hline Recover & SB4-SME-1 & 9,500 & 41.2 & 33.7 & 3.05 & 2.12 & -30.5 & np & SRNL-STI-2011-674 & DWPF Simulated Melter Feed \\
\hline Recover & 09-SB5-23 SME & 11,221 & 49.6 & 35.1 & 24.4 & 16.5 & -32.4 & 7.37 & SRNL-STI-2009-697 & DWPF Simulated Melter Feed \\
\hline Recover & SB4-SME-1 & 14,000 & 41.2 & 33.7 & 3.05 & 2.06 & -32.5 & np & SRNL-STI-2011-674 & DWPF Simulated Melter Feed \\
\hline Recover & SB4-SME-3 & 5,500 & 46.7 & 38.4 & 7.49 & 5.04 & -32.7 & np & SRNL-STI-2011-674 & DWPF Simulated Melter Feed \\
\hline Recover & RuRhHg 1-9 SME & 16,121 & 51.55 & 42 & 13.4 & 9 & -32.8 & 4.7 & SRNL-STI-2009-697 & DWPF Simulated Melter Feed \\
\hline Recover & SB6-1,2,3,4 SME & 16,145 & 46.9 & 37.2 & 2.2 & 1.41 & -35.9 & 6.02 & SRNL-STI-2009-697 & \begin{tabular}{|l|} 
DWPF Simulated Melter Feed \\
\end{tabular} \\
\hline Recover & 08-SB5-12/13 SME & 16,041 & 44.7 & 34.4 & 6.6 & 4.2 & -36.4 & 6.27 & SRNL-STI-2009-697 & DWPF Simulated Melter Feed \\
\hline Recover & 09-SB5-24 SME & 16,000 & 48.8 & 34.5 & 5.22 & 3.28 & -37.2 & np & SRNL-STI-2009-697 & DWPF Simulated Melter Feed \\
\hline Recover & SB4-SME-4 & 9,500 & 49.2 & 40.1 & 13.5 & 8.4 & -37.8 & np & SRNL-STI-2011-674 & DWPF Simulated Melter Feed \\
\hline Recover & SB4-SME-3 & 14,000 & 46.7 & 38.4 & 7.49 & 4.65 & -37.9 & np & SRNL-STI-2011-674 & DWPF Simulated Melter Feed \\
\hline Recover & 09-SB5-23 SME & 16,108 & 49.6 & 35.1 & 24.4 & 15.1 & -38.1 & 7.37 & SRNL-STI-2009-697 & DWPF Simulated Melter Feed \\
\hline Recover & SB4-SME-3 & 9,500 & 46.7 & 38.4 & 7.49 & 4.37 & -41.7 & $\mathrm{np}$ & SRNL-STI-2011-674 & DWPF Simulated Melter Feed \\
\hline Recover & SB4-SME-4 & 14,000 & 49.2 & 40.1 & 13.5 & 7.6 & -43.7 & $\mathrm{np}$ & SRNL-STI-2011-674 & DWPF Simulated Melter Feed \\
\hline Sodium Metasilicate & $\begin{array}{l}\text { RuRhHg 1-9 Decanted } \\
\text { SME }\end{array}$ & 1,019 & np & np & 72.5 & 70.1 & -3.3 & 4.59 & SRNL-STI-2009-697 & DWPF Simulated Melter Feed \\
\hline Sodium Metasilicate & $\begin{array}{l}\text { RuRhHg 1-9 Decanted } \\
\text { SME }\end{array}$ & 3,523 & np & np & 72.5 & 61.8 & -14.8 & 4.59 & SRNL-STI-2009-697 & DWPF Simulated Melter Feed \\
\hline Sodium Metasilicate & $\begin{array}{l}\text { RuRhHg 1-9 Decanted } \\
\text { SME }\end{array}$ & 7,044 & np & np & 72.5 & 58.9 & -18.8 & 4.59 & SRNL-STI-2009-697 & DWPF Simulated Melter Feed \\
\hline
\end{tabular}




\begin{tabular}{|c|c|c|c|c|c|c|c|c|c|c|}
\hline Modifier & Sample & $\begin{array}{l}\text { Mod conc, } \\
\text { ppm }\end{array}$ & $\begin{array}{c}\text { Total Solids, } \\
\%\end{array}$ & UDS, \% & \begin{tabular}{|c|} 
Yield \\
Stress \\
no Mod, \\
Pa \\
\end{tabular} & $\begin{array}{c}\text { Yield } \\
\text { Stress w } \\
\text { Mod, Pa }\end{array}$ & $\begin{array}{l}\text { Calc \% } \\
\text { Change }\end{array}$ & pH & Ref & Category \\
\hline Sodium Metasilicate & $\begin{array}{l}\text { RuRhHg 1-9 Decanted } \\
\text { SME }\end{array}$ & 10,002 & np & np & 72.5 & 65.2 & -10.1 & 4.59 & SRNL-STI-2009-697 & DWPF Simulated Melter Feed \\
\hline Sodium Polyphosphate & AZ-101 & 1,000 & $\mathrm{np}$ & np & 3.22 & 2.93 & -9.0 & 12.23 & SRNL-GPD-2004-40 & Hanford Simulated Waste \\
\hline Sodium Polyphosphate & FIU AZ-101 & 1,000 & np & np & 10.36 & 8.66 & -16.4 & 9.85 & \begin{tabular}{|l|} 
SRNL-GPD-2004-40 \\
\end{tabular} & Hanford Simulated Waste \\
\hline \begin{tabular}{|l|} 
Sodium Pyrophosphate \\
Tetrabasic \\
\end{tabular} & SB6-1,2,3,4 SME & 1,007 & 46.9 & 37.2 & 2.2 & 2.44 & 10.9 & 6.02 & SRNL-STI-2009-697 & DWPF Simulated Melter Feed \\
\hline $\begin{array}{l}\text { Sodium Pyrophosphate } \\
\text { Tetrabasic }\end{array}$ & SB6-1,2,3,4 SME & 7,104 & 46.9 & 37.2 & 2.2 & 2.46 & 11.8 & 6.02 & SRNL-STI-2009-697 & DWPF Simulated Melter Feed \\
\hline \begin{tabular}{|l|} 
Sodium Pyrophosphate \\
Tetrabasic
\end{tabular} & SB6-1,2,3,4 SME & 10,036 & 46.9 & 37.2 & 2.2 & 2.43 & 10.5 & 6.02 & SRNL-STI-2009-697 & DWPF Simulated Melter Feed \\
\hline \begin{tabular}{|l|} 
Sodium Pyrophosphate \\
Tetrabasic
\end{tabular} & 08-SB5-12/13 SME & 1,125 & 44.7 & 34.4 & 6.6 & 7.3 & 10.6 & 6.27 & SRNL-STI-2009-697 & DWPF Simulated Melter Feed \\
\hline \begin{tabular}{|l|} 
Sodium Pyrophosphate \\
Tetrabasic \\
\end{tabular} & 08-SB5-12/13 SME & 3,551 & 44.7 & 34.4 & 6.6 & 7.7 & 16.7 & 6.27 & SRNL-STI-2009-697 & DWPF Simulated Melter Feed \\
\hline \begin{tabular}{|l|} 
Sodium Pyrophosphate \\
Tetrabasic
\end{tabular} & 08-SB5-12/13 SME & 7,078 & 44.7 & 34.4 & 6.6 & 8.2 & 24.2 & 6.27 & SRNL-STI-2009-697 & DWPF Simulated Melter Feed \\
\hline $\begin{array}{l}\text { Sodium Pyrophosphate } \\
\text { Tetrabasic }\end{array}$ & 08-SB5-12/13 SME & 10,075 & 44.7 & 34.4 & 6.6 & 8.4 & 27.3 & 6.27 & SRNL-STI-2009-697 & DWPF Simulated Melter Feed \\
\hline $\begin{array}{l}\text { Sodium Pyrophosphate } \\
\text { Tetrabasic }\end{array}$ & RuRhHg 1-9 SME & 1,031 & 51.55 & 42 & 13.4 & 12.2 & -9.0 & 4.7 & SRNL-STI-2009-697 & DWPF Simulated Melter Feed \\
\hline $\begin{array}{l}\text { Sodium Pyrophosphate } \\
\text { Tetrabasic } \\
\end{array}$ & RuRhHg 1-9 SME & 3,488 & 51.55 & 42 & 13.4 & 14.8 & 10.4 & 4.7 & SRNL-STI-2009-697 & DWPF Simulated Melter Feed \\
\hline $\begin{array}{l}\text { Sodium Pyrophosphate } \\
\text { Tetrabasic }\end{array}$ & RuRhHg 1-9 SME & 7,002 & 51.55 & 42 & 13.4 & 19.7 & 47.0 & 4.7 & SRNL-STI-2009-697 & DWPF Simulated Melter Feed \\
\hline $\begin{array}{l}\text { Sodium Pyrophosphate } \\
\text { Tetrabasic }\end{array}$ & RuRhHg 1-9 SME & 9,983 & 51.55 & 42 & 13.4 & 23.9 & 78.4 & 4.7 & SRNL-STI-2009-697 & DWPF Simulated Melter Feed \\
\hline Sugar & $\begin{array}{l}\text { RuRhHg 1-9 Decanted } \\
\text { SME }\end{array}$ & 1,012 & np & np & 72.5 & 69 & -4.8 & 4.59 & SRNL-STI-2009-697 & DWPF Simulated Melter Feed \\
\hline Sugar & $\begin{array}{l}\text { RuRhHg 1-9 Decanted } \\
\text { SME }\end{array}$ & 3,511 & np & np & 72.5 & 70.9 & -2.2 & 4.59 & SRNL-STI-2009-697 & DWPF Simulated Melter Feed \\
\hline Sugar & $\begin{array}{l}\text { RuRhHg 1-9 Decanted } \\
\text { SME }\end{array}$ & 7,031 & np & np & 72.5 & 63.8 & -12.0 & 4.59 & SRNL-STI-2009-697 & DWPF Simulated Melter Feed \\
\hline Sugar & $\begin{array}{l}\text { RuRhHg 1-9 Decanted } \\
\text { SME }\end{array}$ & 10,012 & $\mathrm{np}$ & $\mathrm{np}$ & 72.5 & 59.9 & -17.4 & 4.59 & SRNL-STI-2009-697 & DWPF Simulated Melter Feed \\
\hline Taylor Antifoam 747 & \begin{tabular}{|l} 
SB6-1,2,3,4 SME \\
\end{tabular} & 1,013 & 46.9 & 37.2 & 2.2 & 2.57 & 16.8 & 6.02 & SRNL-STI-2009-697 & DWPF Simulated Melter Feed \\
\hline Taylor Antifoam 747 & SB6-1,2,3,4 SME & 6,009 & 46.9 & 37.2 & 2.2 & 2.51 & 14.1 & 6.02 & SRNL-STI-2009-697 & DWPF Simulated Melter Feed \\
\hline Taylor Antifoam 747 & SB6-1,2,3,4 SME & 11,143 & 46.9 & 37.2 & 2.2 & 2.48 & 12.7 & 6.02 & SRNL-STI-2009-697 & DWPF Simulated Melter Feed \\
\hline Taylor Antifoam 747 & SB6-1,2,3,4 SME & 16,027 & 46.9 & 37.2 & 2.2 & 2.47 & 12.3 & 6.02 & SRNL-STI-2009-697 & DWPF Simulated Melter Feed \\
\hline
\end{tabular}


From: Chun, J.; Poloski, A. P.; Hansen, E. K., Stabilization and control of rheological properties of $\mathrm{Fe} 2 \mathrm{O} / \mathrm{Al}(\mathrm{OH}) 3$-rich colloidal slurries under high ionic strength and $\mathrm{pH}$. Journal of Colloid and Interface Science 2010, 348, 280-288.

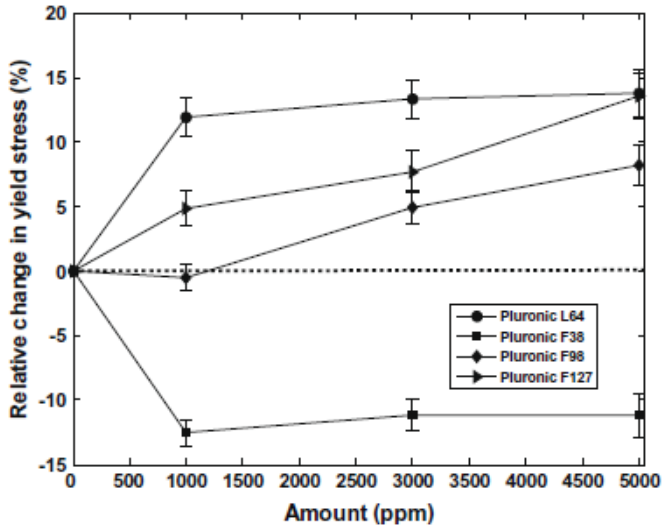

Fig 4. Values of relative change in yield stress for Pluronic surfactants. Four different Pluronic surfactants are used: L64, F38, F98, and F127. Error bars indicat the uncertainty less than $2 \%$ in terms of relative change in yield stress.

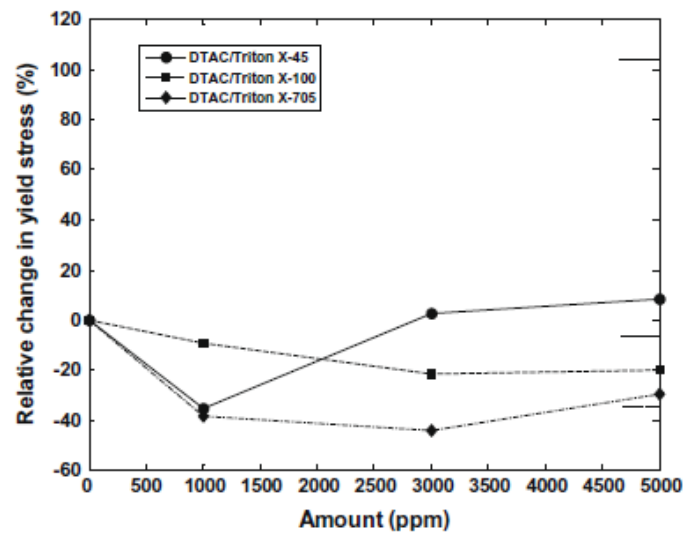

Fig. 6. Values of relative change in yield stress for DTAC/Triton X-45, DTAC/Triton X-100, and DTAC/Triton X-705 combinations. Solid, dashed, and dash-dot lines correspond to values at $5000 \mathrm{ppm}$ only with Triton X-45, Triton X-100, and Triton correspond to values at $5000 \mathrm{ppm}$ only with Triton $X-45$, Triton $X-100$, and Trits
$X-705$, respectively. Note that experimental uncertainties are less than symbols.

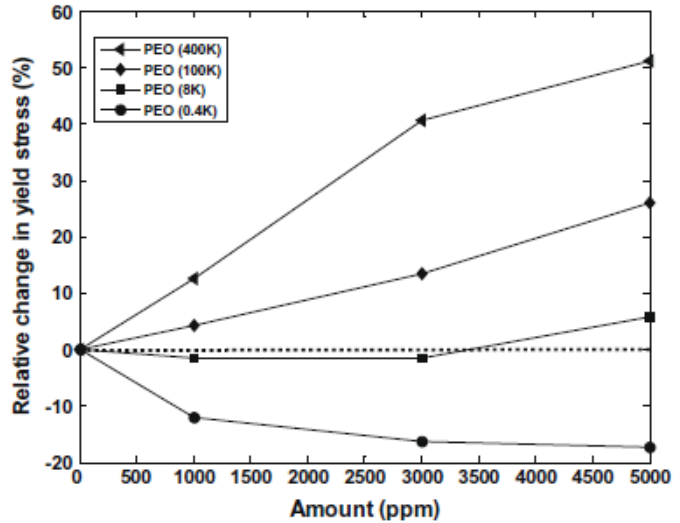

Fig. 5. Values of rel ative change in yield stress for PEO with four different molecular masses: $400,8000,100,000$, and $400,000 \mathrm{~g} / \mathrm{mol}$. Note that experimental uncertainties are less than symbols.

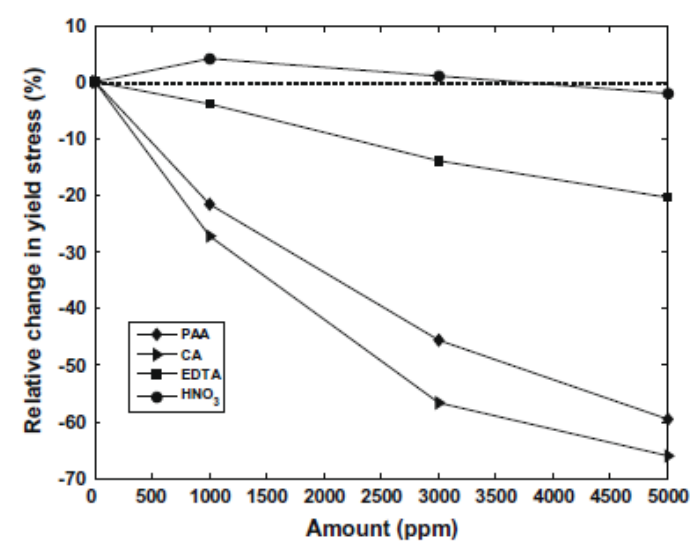

Fig. 7. Values of relative change in yield stress for three weak acids (polyacrylic acid (PAA), citric acid (CA), and ethylenediaminetetraacetic acid (EDTA)) and nitric acid. Note that experimental uncertainties are less than symbols. 
SRNL-STI-2013-00493

Revision 0

Appendix B. Evaluated Rheological Modifiers: Excerpts From Reference Documents 
From: Stone, M. E. Summary of Rheological Modifier Testing on RPP Simulants; SRNL-GPD2004-0040; Savannah River National Laboratory: Aiken, SC, 2004.

Table 1. Rheological Modifiers Tested

\begin{tabular}{|l|l|l|}
\hline Additive & Type & Manufacturer \\
\hline Sodium Polyphosphate & Phosphate polymer & Various \\
\hline Duramax 3005 & Ammonium Polyacrylate & Rohm and Haas \\
\hline Dolapix CE64 & Proprietary Polyacrylate & Zschimmer and Schwartz \\
\hline Alcosperse 149 & Sodium polyacrylate & Alco Chemical \\
\hline Alcosperse 240 & Proprietary Polyacrylate & Alco Chemical \\
\hline Alcosperse 408 & Proprietary Polyacrylate & Alco Chemical \\
\hline Alcosperse 725 & Proprietary Polyacrylate & Alco Chemical \\
\hline EDA Plan 470 & Proprietary Polyacrylate & Ultra Additives \\
\hline EDA Plan 472 & Proprietary Polyacrylate & Ultra Additives \\
\hline Pomosperse AL36 & Proprietary Polyacrylate & Piedmont Chemical Co. \\
\hline Cyanamer P-35 & Proprietary Polyacrylate & Cytec \\
\hline Cyanamer P-70 & Proprietary Polyacrylate & Cytec \\
\hline
\end{tabular}

From: Stone, M. E.; Marinik, A. R.; Marsh, D. M. Rheological Modifier Testing with DWPF Process Slurries; WSRC-TR-2004-00082; Savannah River National Laboratory: Aiken, SC, 2004.

Table 1. Rheological Modifiers Tested

\begin{tabular}{|c|c|c|}
\hline Additive & Type & Manufacturer \\
\hline Sodium Meta-Silicate & Crystalized silicate & Various \\
\hline Sodium Polyphosphate & Phosphate polymer & Various \\
\hline Darvan 7 & Polymethylacrylate, anionic & Vanderbilt Co. Inc. \\
\hline Duramax 3005 & Ammonium Polyacrylate & Rohm and Haas \\
\hline Dolapix CE64 & Proprietary Ethylene Glycol & Zschimmer and Schwartz \\
\hline Disperse-Ayd W22 & Proprietary Polyacrylate & Elementis Specialties \\
\hline Disperse-Ayd W28 & Proprietary Polyacrylate & Elementis Specialties \\
\hline Disperse-Ayd W30 & Proprietary Polyacrylate & Elementis Specialties \\
\hline Disperse-Ayd W39 & Proprietary Polyacrylate & Elementis Specialties \\
\hline Alcosperse 149 & Sodium polyacrylate & Alco Chemical \\
\hline Alcosperse 240 & Proprietary Polyacrylate & Alco Chemical \\
\hline Alcosperse 408 & Proprietary Polyacrylate & Alco Chemical \\
\hline Alcosperse 725 & Proprietary Polyacrylate & Alco Chemical \\
\hline EDA Plan 470 & Proprietary Polyacrylate & Ultra Additives \\
\hline EDA Plan 472 & Proprietary Polyacrylate & Ultra Additives \\
\hline Pomosperse AL36 & Proprietary Polyacrylate & Piedmont Chemical Co. \\
\hline Cyanamer P-35 & Proprietary Polyacrylate & Cytec \\
\hline Cyanamer P-70 & Proprietary Polyacrylate & Cytec \\
\hline Sugar & Glucose & Various \\
\hline
\end{tabular}


From: Hansen, E. K. 2011 EM/SRNL Rheology Modifiers Summary Report; SRNL-STI-201100670; Savannah River National Laboratory: Aiken, SC, 2011.

\begin{tabular}{|c|c|c|c|c|c|c|c|}
\hline Vendor & $\begin{array}{l}\text { Chemical } \\
\text { Name }\end{array}$ & Components of Admixture & Structure & $w t \%$ & $\mathrm{pH}$ & Density & NOTES \\
\hline $\begin{array}{c}\text { Aldrich } \\
\text { Chemical Co. }\end{array}$ & $\begin{array}{l}\text { Poly (Acrylic } \\
\text { Acid) }\end{array}$ & $\begin{array}{l}\text { Polyacrylic acid, average } \\
\text { molecular weight is } 1800\end{array}$ & & $100 \%$ & - & - & Solid \\
\hline $\begin{array}{l}\text { ACROS } \\
\text { Organics }\end{array}$ & $\begin{array}{l}\text { Poly(acrylic } \\
\text { acid), } 63 \text { wt\% } \\
\text { aqueous } \\
\text { solution }\end{array}$ & $\begin{array}{c}37 \% \mathrm{H}_{2} 0,47 \mathrm{wt} \% 2000 \mathrm{MW} \\
\text { polyacrylic acid, } 16 \% \text { sodium } \\
\text { polyacrylate }\end{array}$ & & $60 \%$ & $\begin{array}{l}2.2- \\
3.0\end{array}$ & 1.230 & Liquid \\
\hline $\begin{array}{l}\text { ACROS } \\
\text { Organics }\end{array}$ & $\begin{array}{l}\text { Poly(acrylic } \\
\text { acid), } 50 \text { wt } \% \\
\text { aqueous } \\
\text { solution }\end{array}$ & $\begin{array}{c}50 \% \mathrm{H}_{2} 0,37 \mathrm{wt} \% 2000 \mathrm{MW} \\
\text { polyacrylic acid, } 13 \% \text { sodium } \\
\text { polyacrylate }\end{array}$ & & $50 \%$ & $\begin{array}{l}2.2- \\
3.0\end{array}$ & 1.180 & Liquid \\
\hline $\begin{array}{c}\text { NOAH } \\
\text { Technologies }\end{array}$ & $\begin{array}{l}\text { Citric Acid, } \\
\text { anhydrous }\end{array}$ & $\mathrm{C}_{6} \mathrm{H}_{8} \mathrm{O}_{7}$ & & $100 \%$ & - & - & Solid \\
\hline $\begin{array}{l}\text { Aldrich } \\
\text { Chemical Co }\end{array}$ & $\begin{array}{c}\text { Glycolic Acid, } \\
\text { technical, } 70 \\
\text { wt } \%\end{array}$ & $\mathrm{C}_{2} \mathrm{H}_{4} \mathrm{O}_{3}$ & & 70 & - & 1.25 & Liquid \\
\hline \multirow[t]{2}{*}{ W. R. Grace } & \multirow[t]{2}{*}{ Recover ${ }^{*}$} & $\begin{array}{c}\text { Tetrahydro-3,5-dimethyl-2H- } \\
\text { 1,3,4-thiadiazine-2-thione }\end{array}$ & & 0.004 & \multirow[t]{2}{*}{$5-8$} & \multirow[t]{2}{*}{$1.1-1.2$} & \multirow[t]{2}{*}{ Liquid } \\
\hline & & Sodium Gluconate, $\mathrm{NaC}_{4} \mathrm{H}_{\|} \mathrm{O}_{2}$ & & - & & & \\
\hline
\end{tabular}

From: Kay, E. D.; T. B. Calloway, J.; Koopman, D. C.; Brigmon, R. L.; Eibling, R. E. Rheology Modifiers for Radioactive Waste Slurries; WSRC-MS-2003-00136; Westinghouse Savannah River Company: Aiken, SC, 2003.

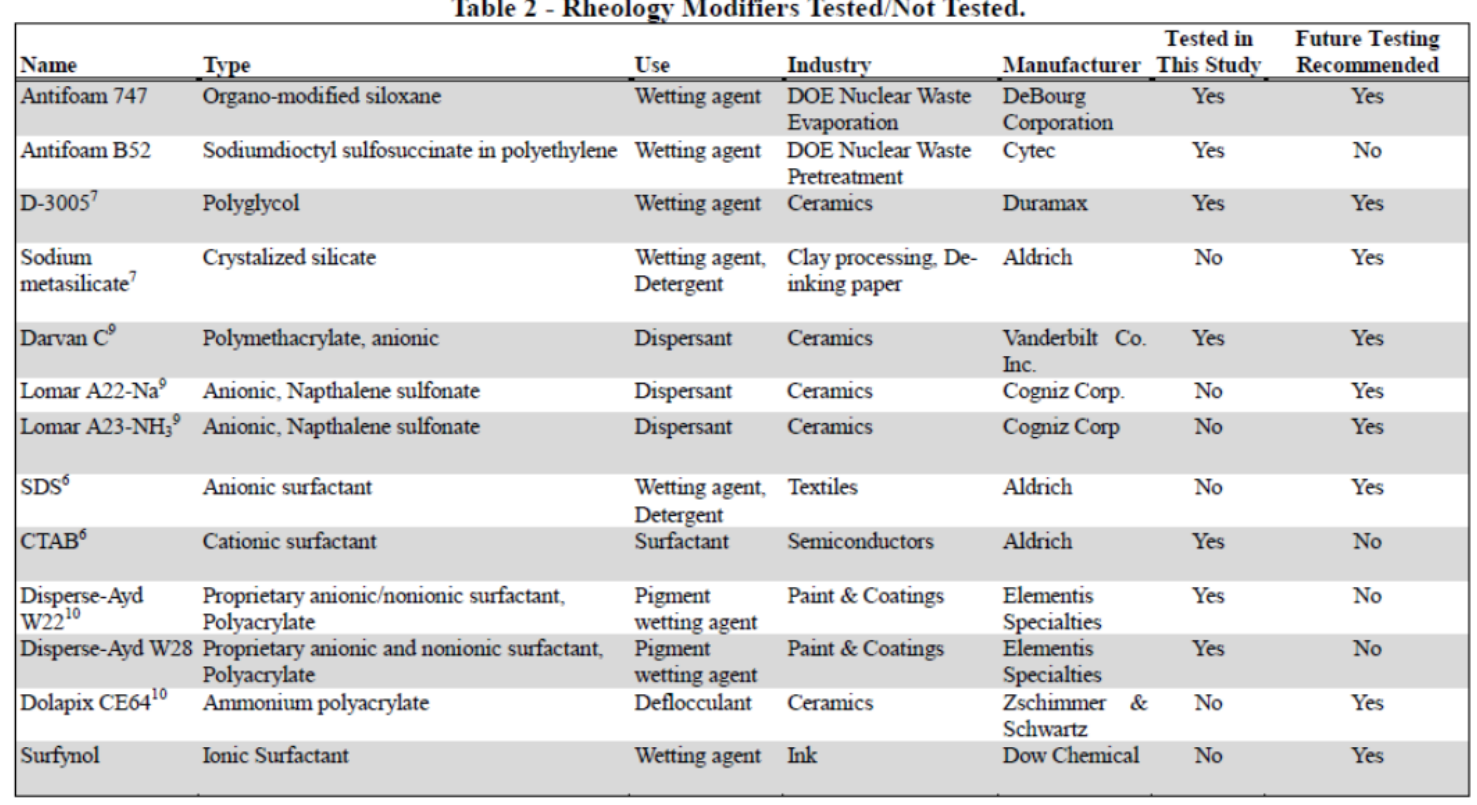


From: Hansen, E. K. Summary of 2009 Rheology Modifier Program; SRNL-STI-2009-00697; Savannah River National Laboratory: Aiken, SC, 2009.

Table 3-15 Rheology Modifiers Used in SRNL FY-2009 Testing

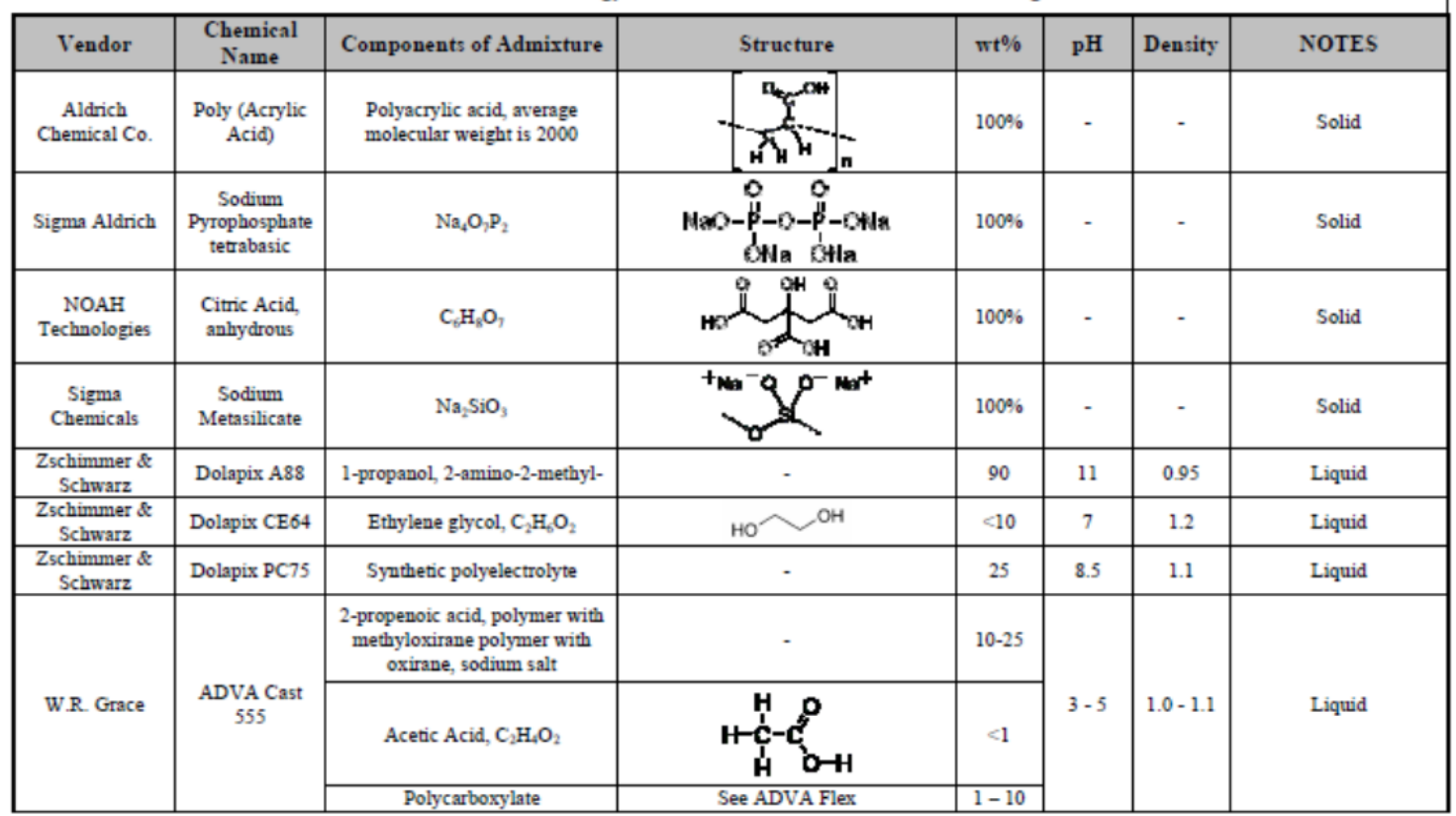

Table 3-15 Rheology Modifiers Used in SRNL FY-2009 Testing

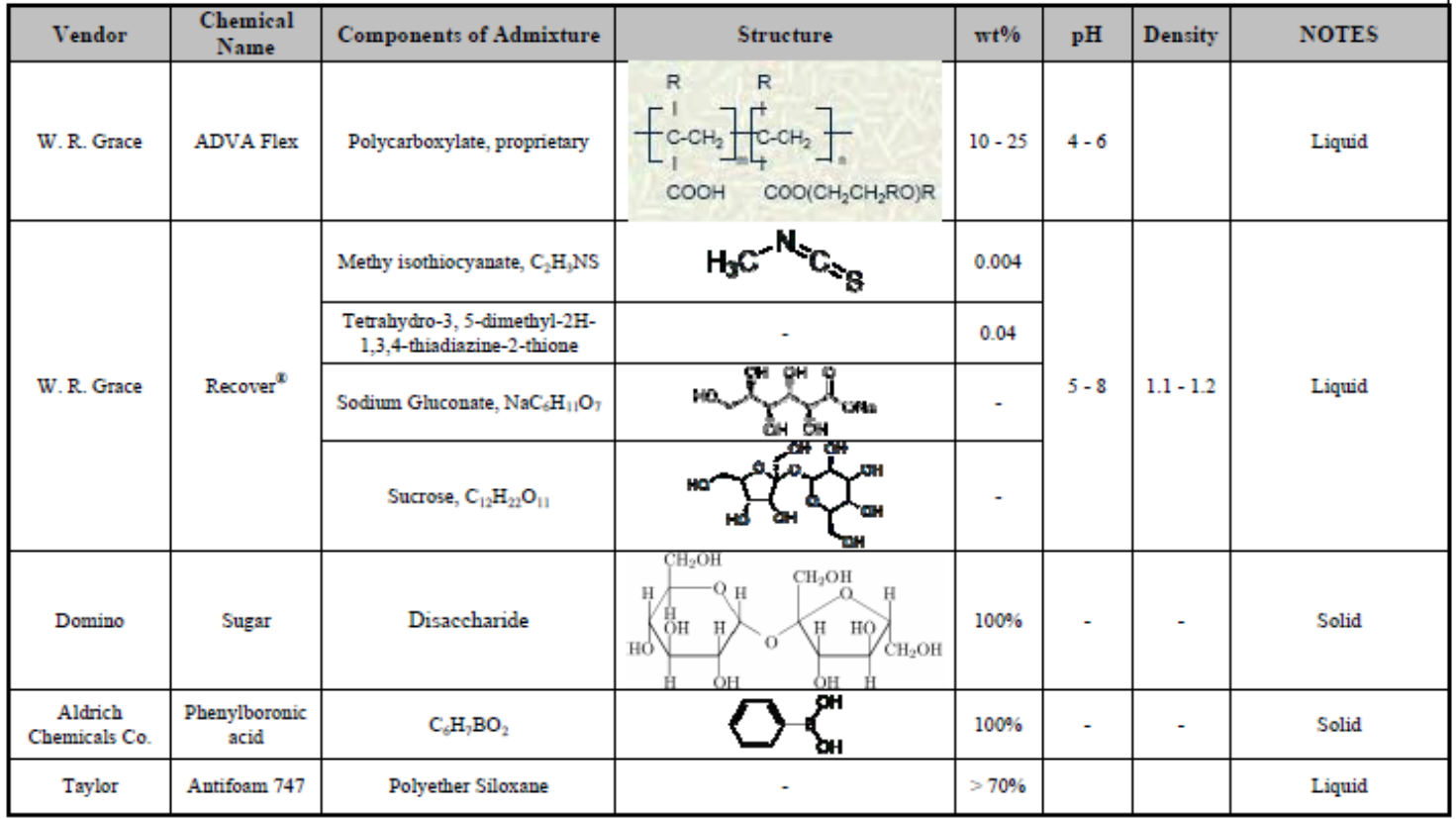


From: Chun, J.; Poloski, A. P.; Hansen, E. K., Stabilization and control of rheological properties of Fe2O3/Al(OH)3-rich colloidal slurries under high ionic strength and $\mathrm{pH}$. Journal of Colloid and Interface Science 2010, 348, 280-288.

As weak acid-type rheological modifiers, we used polyacrylic acid $\left(M_{w} \sim 1800 \mathrm{~g} / \mathrm{mol}\right)$, citric acid, ethylenediaminetetraacetic acid, oxalic acid, nitrilotriacetic acid, gluconic acid, glycolic acid, iminodiacetic acid, and L-tartaric acid. As nonionic/polymer surfactant-type rheological modifiers, Triton X-45, X-100, X-705 (Dow Chemicals), Pluronic L64, F38, F98 (BASF), and polyethylene oxides with four different molecular masses $(400,8000,100,000$, and $400,000 \mathrm{~g} / \mathrm{mol}$ ) were used. Polyethylene oxide with $8000 \mathrm{~g} / \mathrm{mol}$ was obtained from Dow Chemicals. A cationic surfactant, dodecyltrimethyl-ammonium chloride (DTAC), was used to observe synergetic effects with Triton X-45, X-100, and X-705. Rheological modifiers used in the previous studies, sodium lignosulfonate $\left(M_{w} \sim 52,000 \mathrm{~g} / \mathrm{mol}\right)$, sodium metasilicate, and Duramax D-3005 (Rohm \& Haas), were tested for comparison. Note that Duramax D-3005 is known as an ammonium salt of acrylic polymer. All chemicals were purchased from Sigma-Aldrich unless otherwise indicated. Three different concentrations, 1000, 3000, and $5000 \mathrm{ppm}$ (or $\mu$ g per a gram of slurry), were typically added. 


\section{Distribution:}

SRNL

S. L. Marra, 773-A

T. B. Brown, 773-A

D. R. Click, 999-W

S. D. Fink, 773-A

C. C. Herman, 773-A

E. N. Hoffman, 999-W

F. M. Pennebaker, 773-42A

W. R. Wilmarth, 773-A

Records Administration (EDWS)

E. K. Hansen, 999-W

D. T. Herman, 735-11A

D. K. Peeler, 999-W

M. R. Poirier, 773-42A

S. H. Reboul, 773-42A

M. E. Stone, 999-W

SRR

J. M. Bricker, 704-27S

K. H. Subramanian, 241-156H

PNNL

R. D. Peterson
DOE-ORP

S. L. Charboneau

J. A. Diediker

T. W. Fletcher

B. J. Harp

C. C. Harrington

W. R. Wrzesinski

WTP

A. V. Arakali

S. M. Barnes

P. A. Benson

G. M. Duncan

M. R. Hamlet

J. Markillie

WRPS

S. T. Arm

C. Burrows

P. Certa

T. W. Crawford

W. G. Ramsey

J. G. Reynolds

P. L. Rutland

S. A. Saunders

M. G. Thien 